\title{
Actualización taxonómica de las algas rojas (Rhodophyta) marinas bentónicas del Atlántico mexicano
}

\author{
Taxonomic update of the benthic marine red \\ algae (Rhodophyta) from the Mexican Atlantic
}

Acta Botanica Mexicana

\author{
Annie May Ek García-Garcíal (D) , Ernesto Cabrera-Becerril² (D) , María Luisa Núñez-Resendizz (D) \\ Kurt M. Dreckmann² (D), Abel Sentíes² (D)
}

\section{Resumen:}

Antecedentes y Objetivos: Desde las contribuciones de Dreckmann en 1998 y Ortega et al. en 2001, no se han realizado otros trabajos compilatorios para el Atlántico mexicano en los que se consideren modificaciones y actualizaciones taxonómicas para la flora ficológica de la zona. El objetivo de este trabajo fue presentar un listado actualizado de las algas rojas de la costa mexicana del Golfo de México y el Caribe mexicano, en el que se consideran los nuevos registros para la región a partir de los trabajos mencionados hasta la fecha.

Métodos: La información de las especies se obtuvo revisando la mayor cantidad de literatura publicada para el área de estudio, desde 2001 a la fecha y algunos registros previos no considerados antes, así como las bases de datos del Herbario Metropolitano UAMIZ. La sinonimia y el estatus nomenclatural de cada nombre, así como el sistema de clasificación utilizado, fueron revisados en AlgaeBase.

Resultados clave: Se obtuvo un listado florístico con 451 especies y 13 categorías infraespecíficas de algas rojas distribuidas en 4 clases, 23 órdenes, 52 familias y 171 géneros. Para cada especie se menciona su estado nomenclatural actualmente aceptado, su sinonimia y su distribución. Además, se resaltan con un asterisco aquellos registros antiguos (previos al 2002), así como su distribución por ambiente.

Conclusiones: El presente listado reveló dos grandes cambios a la previamente conocida flora de las algas rojas de la zona de estudio. El primero de ellos se encuentra en el terreno de la nomenclatura, e implica una modificación a la clasificación de los taxones (familias, géneros, especies). El segundo repercute en el número de especies presentes. Dado lo anterior, es evidente la necesidad de un monitoreo florístico constante a lo largo del litoral del Atlántico mexicano.

Palabras clave: distribución, diversidad, florística, nomenclatura.

\section{Abstract:}

Background and Aims: Since the contributions of Dreckmann in 1998, and Ortega et al. in 2001, there are no other integrative studies for the Mexican Atlantic seaweeds in which modifications and taxonomic actualizations were considered for this regional phycological flora. Hence, our main objective was to present an update for the marine benthic red algae from the Mexican coast of the Gulf of Mexico, and the Mexican Caribbean Sea, in which all the new records are included for the region since the contributions mentioned above.

Methods: The information about the species was obtained through the analysis of all the specialized literature published for the region since 2001, as well as some previous records not considered before, and a revision of the databases of the UAMIZ Metropolitan Herbarium. Synonymy and nomenclatural status, as well as the classification system used, were revised in AlgaeBase.

Key results: A checklist composed of 451 species and 13 infraespecific categories of red algae was obtained; these where distributed in 4 classes, 23 orders, 52 families, and 171 genera. The currently accepted name, synonymy, and distribution are mentioned for each species. An asterisk indicates previous records (prior to 2002), as well as its distribution in different habitats.

Conclusions: The present contribution revealed two main changes to the previously known flora of the red algae from the study area. The first one falls in the domain of nomenclature, and implies a modification in their classification (families, genera, and species). The second one has an effect on the number of species present. As a consequence, it is evident that constant floristic monitoring is required along the coasts of the Mexican Atlantic.

Key words: distribution, diversity, floristics, nomenclature.

${ }^{1}$ Universidad Nacional Autónoma de México, Facultad de Ciencias, Coyoacán, Apdo. postal 70-474, 04510 Cd. Mx., México.

${ }^{2}$ Universidad Autónoma Metropolitana-Iztapalapa, Departamento de Hidrobiología, Apdo. postal 55-535, 09340 Cd. Mx., México.

${ }^{3}$ Autor para la correspondencia: mlnr@ciencias.unam. $\mathrm{mx}$
Recibido: 13 de diciembre de 2020. Revisado: 10 de marzo de 2020

Aceptado por Marie-Stéphanie Samain: 15 de abril de 2020.

Publicado Primero en línea: 8 de junio de 2020.

Publicado: Acta Botanica Mexicana 127(2020).
Citar como: García-García, A. M. E., E. Cabrera-Becerril, M. L. Núñez-Resendiz, K. M. Dreckmann y A. Sentíes. 2020. Actualización taxonómica de las algas rojas (Rhodophyta) marinas bentónicas del Atlántico mexicano. Acta Botanica Mexicana 127: e1677. DOI: 10.21829/abm127.2020.1677 c) (7) Este es un artículo de acceso abierto bajo la licencia Creative Commons 4.0 Atribución-No Comercial (CC BY-NC 4.0 Internacional). 


\section{Introducción}

El Atlántico Mexicano (AM) comprende el litoral mexicano del Golfo de México y el Caribe mexicano, su longitud aproximada es de 3294 km de línea de costa y 1080 km adicionales de los bordes insulares (Pedroche y Sentíes, 2003). Esta extensión cuenta con una alta heterogeneidad de ambientes propicios para el establecimiento de diferentes comunidades acuáticas en las que predomina el grupo de las algas rojas (Pedroche et al., 2009; Sentíes y Dreckmann, 2011). Hasta ahora, en la región han sido registradas cerca de 350 especies distribuidas en 15 familias y 80 géneros (Núñez-Resendiz et al., 2019a), lo que representa 34.5\% de la diversidad total en todo el Atlántico, en donde se repor$\tan 1015$ especies (Wynne, 2017) y $4.8 \%$ de la diversidad a nivel mundial con aproximadamente 7278 especies aceptadas taxonómicamente en la actualidad (Guiry y Guiry, 2020).

Trabajos de índole florística han permitido conocer bien la flora de la región (Mendoza-González et al., 2016; Núñez-Resendiz et al., 2019b; Vázquez-Delfin et al., 2019). Los trabajos publicados antes de 2002 para el Golfo de México y el Caribe mexicano se encuentran reunidos en dos catálogos importantes: el de Dreckmann (1998) y el de Ortega et al. (2001).

Aunque los listados florísticos constituyen el primer acercamiento al conocimiento de la diversidad para cualquier región determinada, en su conjunto, dichos listados permiten la realización de otro tipo de estudios más integrales como los ecológicos o biogeográficos. Entre estos trabajos destacan los que determinan riqueza y composición florística (Mateo-Cid y Mendoza-González, 1991; Mendoza-González y Mateo-Cid, 1992; Dreckmann et al., 1996; Mateo-Cid et al., 1996, 2006, 2013a,b; Sentíes y Dreckmann, 2011, 2013; Tapia-Silva et al., 2015; Mendoza-González et al., 2017), estudios de afinidad florística mediante la comparación de dos o más regiones (Dreckmann y Pérez-Hernández, 1994; Orozco-Vega y Dreckmann, 1995), reconocimiento de especies endémicas o invasoras (Hernández et al., 2017), determinación de patrones de distribución de taxa (Dreckmann, 2012; Ortegón-Aznar y Aguilar-Perera, 2014; Hernández et al., 2017; Vilchis et al., 2018), localización de áreas de concentración de biota, áreas de endemismo o realización de propuestas de conservación (López-Valdez, 2016; Vilchis et al., 2018).

Las algas rojas son, dentro de las macroalgas, el grupo que desde un enfoque molecular ha sido más trabajado actualmente (Díaz-Larrea et al., 2016), por lo que el conocimiento de su diversidad en el AM se ha incrementado considerablemente (Dreckmann y Sentíes, 2014; Sentíes y Dreckmann, 2014; Núñez-Resendiz et al., 2017a; VázquezDelfín et al., 2019). Sin embargo, también su taxonomía en todos los niveles jerárquicos ha sido modificada drásticamente; existen numerosos cambios nomenclaturales, principalmente, al interior de muchos de los órdenes y familias registrados para la región. Por ejemplo, muchos nombres han caído en desuso (García-Soto y López-Bautista, 2018; Gurgel et al., 2018), otros han sido detectados como mal aplicados (Boo et al., 2018; Guiry et al., 2018), muchas especies han sido puestas en sinonimia (Sentíes et al., 2009; Núñez-Resendiz et al., 2015) y otras han sido reubicadas en nuevos géneros (Sentíes et al., 2015; Collado-Vides et al., 2017; Núñez-Resendiz et al., 2019c). Como consecuencia, la distribución de algunas especies ha quedado delimitada a ciertas regiones del mundo con lo que muchos registros para la zona ya no son válidos (Schneider et al., 2010, 2018; Boo et al., 2018; García-Soto y López-Bautista, 2018), o nuevos géneros y especies han sido descritas (Mateo-Cid et al., 2014a,b, 2016; Sentíes et al., 2016; Núñez-Resendiz et al., 2017b, 2020; Dreckmann et al., 2018).

Desde las contribuciones de Dreckmann (1998) y Ortega et al. (2001) no se han realizado otros trabajos compilatorios para el AM en los que se consideren las modificaciones y actualizaciones taxonómicas anteriormente referidas para la flora de la zona. Por lo tanto, la presente contribución tiene como objetivo principal presentar un listado actualizado de las algas rojas del Golfo de México y el Caribe mexicano, en el que se consideren todos los nuevos registros para la región, desde 2001 a la fecha, incluyendo actualizaciones y notas taxonómicas en referencia al estatus de cada nombre.

\section{Materiales y Métodos}

La información de las especies de algas rojas registradas en la costa mexicana del Golfo de México y el Caribe mexicano se obtuvo de la revisión de la mayor cantidad de literatura 
publicada para la región (Apéndice 1), desde 2001 a la fecha, incluyendo algunas publicaciones previas a 2001 que no fueron consideradas en el catálogo de Ortega et al. (2001). Adicionalmente, dicha revisión fue completada con la consulta de las bases de datos del Herbario Metropolitano UAMIZ (Thiers, 2020). Fueron revisados de acuerdo con la base de datos AlgaeBase la sinonimia y el estatus nomenclatural de cada nombre, así como el sistema de clasificación utilizado (Guiry y Guiry, 2020).

\section{Resultados}

Se obtuvo un listado florístico con 451 especies y 13 categorias infraespecificas de algas rojas distribuidas en 4 clases, 23 órdenes, 52 familias y 171 géneros. Para cada especie se menciona su estado nomenclatural correcto, sinonimia, distribución por estado y ambiente (litoral, insular o estuario), así como notas taxonómicas cuando se requiere. Se resaltan con un asterisco los registros antiguos, es decir, aquellos cuya última mención fue en el catálogo de Ortega et al. (2001), y que no han sido reportados posteriormente a esta obra para la región. Finalmente, se citan los trabajos en los que se registra a cada especie.

\section{Listado florístico}

Bangiales

Bangiaceae

Bangia atropurpurea (Mertens ex Roth) C. Agardh.

Distribución: Tamaulipas: litoral (Ortega et al., 2001); Veracruz: litoral, insular (Ortega et al., 2001; De la Garza-Flores, 2003); Tabasco: estuario, litoral (Mendoza-González et al., 2017); Yucatán: insular (Ortega et al., 2001); Quintana Roo: insular (Mendoza-González y Mateo-Cid, 1992; Ortega et al., 2001).

Bangia fuscopurpurea (Dillwyn) Lyngbye.

Distribución: Tabasco: estuario, litoral (Mendoza-González et al., 2017; Quiroz-González et al., 2018).
Pyropia leucosticta (Thuret) Neefus \& J. Brodie.

= Porphyra leucosticta Thuret.

Distribución: Tamaulipas: litoral (Ortega et al., 2001; Vargas López et al., 2004, ambos como Porphyra leucosticta); Campeche: litoral (Mateo-Cid et al., 2013b).

Acrochaetiales

Acrochaetiaceae

*Acrochaetium affine M. Howe \& Hoyt.

Distribución: Tamaulipas: litoral (Ortega et al., 2001).

*Acrochaetium antillarum W.R. Taylor.

Distribución: Quintana Roo: insular (Ortega et al., 2001).

*Acrochaetium crassipes (Børgesen) Børges.

三 Kylinia crassipes (Børgesen) Kylin.

Distribución: Veracruz: litoral (Sánchez-Rodríguez, 1980, como Kylinia crassipes).

Acrochaetium flexuosum Vickers.

Distribución: Tamaulipas: litoral (Ortega et al., 2001); Tabasco: litoral (Mendoza-González et al., 2017); Yucatán: estuario (Ortegón-Aznar et al., 2001; Mendoza-González et al., 2016); Quintana Roo: litoral, insular (Mateo-Cid y Mendoza-González, 1991; Ortega et al., 2001; Mendoza-González et al., 2016).

\section{Acrochaetium globosum Børgesen.}

三 Audouinella globosa (Børgesen) Garbary.

Distribución: Quintana Roo: insular (Mateo-Cid y Mendoza-González, 1991, como Audouinella globosa; Ortega et al., 2001; Mendoza-González et al., 2016).

*Acrochaetium hoytii Collins.

Distribución: Tamaulipas: litoral (Ortega et al., 2001). 
Acrochaetium microscopicum (Nägeli ex Kützing) Nägeli. 三 Audouinella microscopica (Nägeli ex Kützing) Woelkerling.

Distribución: Tamaulipas: litoral (Ortega et al., 2001); Veracruz: litoral, insular (Mateo-Cid et al., 1996, como Audouinella microscopica; Ortega et al., 2001; De la Garza-Flores, 2003; Godínez-Ortega et al., 2009, como Audouinella microscopica); Tabasco: litoral (MendozaGonzález et al., 2017); Campeche: litoral (Mateo-Cid et al., 2013a,b; Mendoza-González et al., 2013); Yucatán: litoral (Mendoza-González et al., 2016); Quintana Roo: litoral (Mendoza-González et al., 2016).

\section{Acrochaetium pulchellum Børgesen.}

ミKylinia pulchella (Børgesen) Papenfuss.

Distribución: Veracruz: estuario, litoral (Sánchez-Rodríguez, 1980, como Kylinia pulchella; Ortega et al., 2001; De la Garza-Flores, 2003).

Acrochaetium sagraeanum (Montagne) Bornet.

Distribución: Veracruz: litoral (Ortega et al., 2001; De la Garza-Flores, 2003); Tabasco: litoral (Mendoza-González et al., 2017).

\section{Acrochaetium sancti-thomae Børgesen.}

Distribución: Veracruz: litoral (Sánchez-Rodríguez, 1980; Ortega et al., 2001; De la Garza-Flores, 2003); Tabasco: litoral (Mendoza-González et al., 2017).

Acrochaetium secundatum (Lyngbye) Nägeli.

Distribución: Tabasco: litoral (Mendoza-González et al., 2017).

\section{*Acrochaetium unipes Børgesen.}

Distribución: Quintana Roo: litoral, insular (Ortega et al., 2001).
Acrosymphytales

Acrosymphytaceae

Acrosymphyton caribaeum (J. Agardh) Sjöstedt.

Distribución: Quintana Roo: litoral, insular (Mateo-Cid et al., 2006; Mendoza-González et al., 2007; Cetz-Navarro et al., 2008).

Bonnemaisoniales

Bonnemaisoniaceae

Asparagopsis taxiformis (Delile) Trevisan.

Distribución: Veracruz: litoral, insular (Ortega et al., 2001; De la Garza-Flores, 2003; Luna-Ortega y De la Cruz-Francisco, 2017); Tabasco: litoral (Sentíes y Dreckmann, 2013; Quiroz-González et al., 2018); Campeche: litoral (MateoCid et al., 2013a, b); Yucatán: insular (Ortega et al., 2001); Quintana Roo: estuario, litoral, insular (Mateo-Cid y Mendoza-González, 1991; Mendoza-González y Mateo-Cid, 1992; Collado-Vides y González-González, 1995; Dreckmann et al., 1996; Ortega et al., 2001; Quan-Young et al., 2006; Mateo-Cid et al., 2006; Mendoza-González et al., 2007).

\section{Naccariaceae}

Naccaria antillana W.R. Taylor.

Distribución: Quintana Roo: litoral (Cetz-Navarro et al., 2008).

\section{Ceramiales}

Callithamniaceae

Aglaothamnion boergesenii (Aponte \& D.L. Ballantine) L'Hardy-Halos \& Rueness. 
Distribución: Tabasco: estuario, litoral (Ortega et al., 2001; Mateo-Cid et al., 2003; Sentíes y Dreckmann, 2013; Mendoza-González et al., 2017; Quiroz-González et al., 2018); Campeche: litoral (Mateo-Cid et al., 2013a); Quintana Roo: litoral, insular (Mateo-Cid et al., 2003, 2006; Quan-Young et al., 2006; Cetz-Navarro et al., 2008).

Aglaothamnion cordatum (Børgesen) Feldmann-Mazoyer. इCallithamnion cordatum Børgesen.

Distribución: Tamaulipas: litoral (Ortega et al., 2001; Mateo-Cid et al., 2003); Veracruz: litoral (Ortega et al., 2001; De la Garza-Flores, 2003; Mateo-Cid et al., 2003); Campeche: litoral (Ortega et al., 2001; Mateo-Cid et al., 2003, 2013b; Mendoza-González et al., 2013; Yucatán: litoral (Ortega et al., 2001; Mateo-Cid et al., 2003; Ortegón-Aznar et al., 2009); Quintana Roo: litoral, insular (Mendoza-González y Mateo-Cid, 1992, como Callithamnion cordatum; Dreckmann et al., 1996; Ortega et al., 2001; Mateo-Cid et al., 2003, 2006).

Aglaothamnion felipponei (Howe) Aponte, Ballantine \& J.N. Norris.

Distribución: Quintana Roo: litoral, insular (Quan-Young et al., 2006; Cetz-Navarro et al., 2008).

Aglaothamnion halliae (Collins) Aponte, D.L. Ballantine \& J.N. Norris.

ECallithamnion halliae Collins.

Distribución: Veracruz: litoral, insular (Ortega et al., 2001; De la Garza-Flores, 2003; Núñez-Resendiz, 2009); Yucatán: insular (Ortega et al., 2001); Quintana Roo: litoral, insular (Mateo-Cid y Mendoza-González, 1991; Dreckmann et al., 1996, ambos como Callithamnion halliae; Ortega et al., 2001; Quan-Young et al., 2006).

Aglaothamnion herveyi (M. Howe) Aponte, D.L. Ballantine, \& J.N. Norris.

Distribución: Quintana Roo: litoral (Mateo-Cid et al., 2003; Cetz-Navarro et al., 2008).
*Aglaothamnion tenuissimum (Bonnemaison) FeldmannMazoyer.

= Callithamnion byssoides Arnott ex Harvey.

Distribución: Quintana Roo: insular (Mateo-Cid y Mendoza-González, 1991, como Callithamnion byssoides).

Aglaothamnion uruguayense (W.R. Taylor) Aponte, D.L. Ballantine \& J.N. Norris.

इCallithamnion uruguayense W.R. Taylor.

Distribución: Quintana Roo: insular (Mateo-Cid y MendozaGonzález, 1991, como Callithamnion uruguayense; Ortega et al., 2001).

Callithamnion corymbosum (Smith) Lyngbye.

Distribución: Veracruz: litoral, insular (Mateo-Cid et al., 2003); Tabasco: litoral (Ramírez-López, 1996; Sentíes y Dreckmann, 2013; Mendoza-González et al., 2017; QuirozGonzález et al., 2018); Campeche: litoral (Mendoza-González et al., 2013); Quintana Roo: litoral, insular (Díaz-Martín y Quan-Young, 2001; Mateo-Cid et al., 2003; Quan-Young et al., 2006; Cetz-Navarro et al., 2008).

Crouania attenuata (C. Agardh) J. Agardh.

Distribución: Campeche: litoral (Ortega et al., 2001; Mendoza-González et al., 2013; Mateo-Cid et al., 2013a); Yucatán: insular (Ortega et al., 2001); Quintana Roo: litoral, insular (Mateo-Cid y Mendoza-González, 1991; MendozaGonzález y Mateo-Cid, 1992; Dreckmann et al., 1996; Ortega et al., 2001; Quan-Young et al., 2006; Mateo-Cid et al., 2006; Mendoza-González et al., 2007).

Crouania mayae Mateo-Cid, Mendoza-González \& Searles.

Distribución: Quintana Roo: litoral (Cetz-Navarro et al., 2008).

Crouania pleonospora W.R. Taylor.

Distribución: Quintana Roo: litoral, insular (Díaz-Martín y Quan-Young, 2001; Cetz-Navarro et al., 2008). 
* Gaillona seposita (Gunnerus) Athanasiadis.

= Dasya arbuscula (R. Brown ex Dillwyn) C. Agardh.

Distribución: Quintana Roo: insular (Mendoza-González y Mateo-Cid, 1992, como Dasya arbuscula).

Gymnothamnion elegans (Schousboe ex C. Agardh) J. Agardh.

Distribución: Yucatán: insular (Ortega et al., 2001); Quintana Roo: insular (Mateo-Cid y Mendoza-González, 1991; Ortega et al., 2001).

\section{Seirospora occidentalis Børgesen.}

Distribución: Campeche: litoral (Mateo-Cid et al., 2013a); Quintana Roo: litoral, insular (Díaz-Martín y Quan-Young, 2001; Mateo-Cid et al., 2003; Quan-Young et al., 2006; Mateo-Cid et al., 2006; Cetz-Navarro et al., 2008).

\section{Spyridia clavata Kützing.}

Distribución: Tamaulipas: litoral (Ortega et al., 2001); Veracruz: litoral, insular (Ortega et al., 2001; De la GarzaFlores, 2003); Tabasco: litoral (Mendoza-González et al., 2017).

\section{Spyridia filamentosa (Wulfen) Harvey.}

Distribución: Tamaulipas: litoral (Ortega et al., 2001; Vilchis et al., 2018); Veracruz: estuario, litoral, insular (Zizumbo Alamilla, 1995; Mateo-Cid et al., 1996; Ortega et al., 2001; De la Garza-Flores, 2003; Núñez-Resendiz, 2009; Vilchis et al., 2018); Tabasco: litoral (Quiroz-González et al., 2018); Campeche: estuario, litoral (Ortega, 1995; Ortega et al., 2001; Mateo-Cid et al., 2013b; Mendoza-González et al., 2013; Vilchis et al., 2018); Yucatán: estuario, litoral, insular (Ortega et al., 2001; OrtegónAznar et al., 2001, 2009; Vilchis et al., 2018); Quintana Roo: estuario, litoral, insular (Mendoza-González y Mateo-Cid, 1992, 2007; Collado-Vides y González-González, 1995; Dreckmann et al., 1996; Ortega et al., 2001; Ma-
teo-Cid et al., 2006; Quan-Young et al., 2006; Vilchis et al., 2018).

*Spyridia hypnoides var. disticha (Børgesen) G.W. Lawson \& D.M. John.

Distribución: Tamaulipas: litoral (Ortega et al., 2001).

Spyridia hypnoides var. hypnoides (Bory) Papenfuss.

Distribución: Tamaulipas: litoral (Ortega et al., 2001); Veracruz: litoral, insular (Ortega et al., 2001; De la Garza-Flores, 2003; De la Cruz-Francisco et al., 2017); Tabasco: litoral (Sentíes y Dreckmann, 2013; Mendoza-González et al., 2017; Quiroz-González et al., 2018); Campeche: litoral (Mateo-Cid et al., 2013b); Yucatán: litoral, insular (Ortega et al., 2001; Ortegón-Aznar et al., 2009); Quintana Roo: litoral, insular (Mateo-Cid y Mendoza-González, 1991; MendozaGonzález y Mateo-Cid, 1992; Dreckmann et al., 1996; Ortega et al., 2001; Quan-Young et al., 2006; Mendoza-González et al., 2007).

\section{Ceramiaceae}

Acrothamnion butlerae (Collins) Kylin.

Distribución: Veracruz: litoral (Núñez-Resendiz, 2009); Quintana Roo: litoral, insular (Ortega et al., 2001).

\section{*Antithamnion antillanum Børgesen.}

Distribución: Quintana Roo: insular (Mateo-Cid y Mendoza-González, 1991; Ortega et al., 2001).

Antithamnion cruciatum (C. Agardh) Nägeli.

Distribución: Quintana Roo: litoral, insular (Dreckmann et al., 1996; Ortega et al., 2001; Mendoza-González et al., 2007).

Antithamnion decipiens (J. Agardh) Athanasiadis.

Distribución: Quintana Roo: litoral, insular (Mateo-Cid et al., 2006; Cetz-Navarro et al., 2008). 
Antithamnionella boergesenii (Cormaci \& G. Furnari) Athanasiadis.

Distribución: Tabasco: litoral (Mendoza-González et al., 2017); Campeche: litoral (Mendoza-González et al., 2013); Quintana Roo: litoral, insular (Mateo-Cid et al., 2006; Mendoza-González et al., 2007; Cetz-Navarro et al., 2008).

*Antithamnionella breviramosa (E.Y. Dawson) Wollaston.

Distribución: Quintana Roo: insular (Ortega et al., 2001).

Antithamnionella elegans (Berthold) J.H. Price \& D.M. John.

Distribución: Tabasco: litoral (Mendoza-González et al., 2017); Campeche: litoral (Mateo-Cid et al., 2013a; Mendoza-González et al., 2013).

Callithamniella tingitana (Schousboe ex Bornet) FeldmannMazoyer.

Distribución: Tabasco: litoral (Mendoza-González et al., 2017); Campeche: litoral (Mateo-Cid et al., 2013a, 2013b); Quintana Roo: insular (Mateo-Cid y Mendoza-González, 1991; Ortega et al., 2001; Mateo-Cid et al., 2006).

Centroceras clavulatum (C. Agardh) Montagne.

Distribución: Tamaulipas: estuario, litoral (Ortega et al., 2001; Vargas López et al., 2004); Veracruz: estuario, litoral, insular (Sánchez-Rodríguez, 1980; Zizumbo Alamilla, 1995; Mateo-Cid et al., 1996; Ortega et al., 2001; De la GarzaFlores, 2003; Núñez-Resendiz, 2009; De la Cruz-Francisco et al., 2017); Tabasco: litoral (Ramírez-López, 1996; Sentíes y Dreckmann, 2013; Quiroz-González et al., 2018); Campeche: estuario, litoral (Ortega, 1995; Ortega et al., 2001; Callejas-Jiménez et al., 2005; Mateo-Cid et al., 2013b; Mendoza-González et al., 2013); Yucatán: estuario, litoral, insular (Ortega et al., 2001; Ortegón-Aznar et al., 2001; 2009); Quintana Roo: estuario, litoral, insular (Mateo-Cid y Mendoza-González, 1991; Mendoza-González y Mateo-Cid, 1992; Collado-Vides y González-González, 1995;
Dreckmann et al., 1996; Ortega et al., 2001; Quan-Young et al., 2006; Mateo-Cid et al., 2006).

\section{Centroceras gasparrinii (Meneghini) Kützing.}

Distribución: Veracruz: litoral (Landa-Cansigno et al., 2019); Tabasco: litoral (Mendoza-González et al., 2017).

Centroceras hyalacanthum Kützing.

Distribución: Tabasco: litoral (Mendoza-González et al., 2017).

\section{Centroceras micracanthum Kützing.}

Distribución: Tabasco: litoral (Mendoza-González et al., 2017); Campeche: litoral (Mateo-Cid et al., 2013b).

Centrocerocolax ubatubensis A.B. Joly.

Distribución: Quintana Roo: insular (Mateo-Cid et al., 2006; Cetz-Navarro et al., 2008).

Ceramium brasiliense A.B. Joly.

Distribución: Campeche: estuario (Ortega et al., 2001); Yucatán: insular (Ortega et al., 2001); Quintana Roo: litoral, insular (Mateo-Cid y Mendoza-González, 1991; MendozaGonzález y Mateo-Cid, 1992; Ortega et al., 2001).

Ceramium brevizonatum var. brevizonatum H.E. Petersen.

Distribución: Veracruz: litoral, insular (De la Garza-Flores, 2003); Yucatán: estuario (Sentíes et al., 2015).

Ceramium brevizonatum var. caraibicum H.E. Petersen \& Børgesen.

Distribución: Tamaulipas: litoral (Ortega et al., 2001); Veracruz: litoral, insular (Ortega et al., 2001; De la Garza-Flores, 2003); Tabasco: litoral (Mendoza-González et al., 2017); Campeche: litoral (Mateo-Cid et al., 2013b); Yucatán: litoral (Ortegón-Aznar et al., 2009); Quintana Roo: estuario, 
insular (Mateo-Cid y Mendoza-González, 1991; MendozaGonzález y Mateo-Cid, 1992; Collado-Vides y GonzálezGonzález, 1995; Ortega et al., 2001; Mateo-Cid et al., 2006).

Ceramium caudatum Setchell \& N.L. Gardner.

Distribución: Veracruz: litoral, insular (Ortega et al., 2001; De la Garza-Flores, 2003).

Ceramium cimbricum f. cimbricum H.E. Petersen.

Distribución: Tamaulipas: estuario, litoral (Ortega et al., 2001); Veracruz: litoral, insular (Ortega et al., 2001; De la Garza-Flores, 2003); Tabasco: litoral (Sentíes y Dreckmann, 2013; Quiroz-González et al., 2018); Yucatán: litoral, insular (Ortega et al., 2001); Quintana Roo: estuario, litoral, insular (Ortega et al., 2001; Mateo-Cid et al., 2006).

Ceramium cimbricum f. flaccidum (H.E. Petersen) G. Furnari \& Serio.

Distribución: Tamaulipas: estuario, litoral (Ortega et al., 2001); Veracruz: litoral, insular (Ortega et al., 2001; De la Garza-Flores, 2003; Luna-Ortega y De la Cruz-Francisco, 2017); Campeche: estuario (Ortega et al., 2001; MendozaGonzález et al., 2013); Yucatán: insular (Ortega et al., 2001).

Ceramium codii (H. Richards) Mazoyer.

Distribución: Tamaulipas: litoral (Ortega et al., 2001); Quintana Roo: estuario, litoral, insular (Mateo-Cid y MendozaGonzález, 1991; Mendoza-González y Mateo-Cid, 1992; Collado-Vides y González-González, 1995; Ortega et al., 2001).

\section{Ceramium corniculatum Montagne.}

Distribución: Veracruz: litoral, insular (Ortega et al., 2001; De la Garza-Flores, 2003; Godínez-Ortega et al., 2009); Campeche: litoral (Ortega et al., 2001; Callejas-Jiménez et al., 2005; Mateo-Cid et al., 2013b); Yucatán: litoral, insular (Ortega et al., 2001; Ortegón-Aznar et al., 2009); Quintana Roo: insular (Ortega et al., 2001).
Ceramium cruciatum Collins \& Hervey.

Distribución: Tamaulipas: litoral (Ortega et al., 2001); Veracruz: insular (Zizumbo Alamilla, 1995; Luna-Ortega y De la Cruz-Francisco, 2017); Tabasco: litoral (Mendoza-González et al., 2017); Campeche: litoral (Ortega et al., 2001; MateoCid et al., 2013b); Yucatán: estuario, litoral, insular (Ortega et al., 2001; Ortegón-Aznar et al., 2001, 2009); Quintana Roo: insular (Mateo-Cid y Mendoza-González, 1991; Mendoza-González y Mateo-Cid, 1992; Ortega et al., 2001; Quan-Young et al., 2006).

\section{*Ceramium dawsonii A.B. Joly.}

Distribución: Quintana Roo: insular (Mendoza-González y Mateo-Cid, 1992; Ortega et al., 2001).

Ceramium deslongchampsii Chauvin ex Duby.

Distribución: Tamaulipas: litoral (Ortega et al., 2001); Campeche: estuario, litoral (Ortega et al., 2001; Mateo-Cid et al., 2013b); Quintana Roo: litoral, insular (Ortega et al., 2001; Mendoza-González et al., 2007).

\section{Ceramium diaphanum (Lightfoot) Roth.}

Distribución: Veracruz: litoral (Ortega et al., 2001; De la Garza-Flores, 2003); Campeche: estuario, litoral (Ortega et al., 2001; Mateo-Cid et al., 2013b); Yucatán: insular (Ortega et al., 2001); Quintana Roo: insular (Ortega et al., 2001).

*Ceramium evermannii Setchell \& N.L. Gardner.

Distribución: Campeche: litoral (Ortega et al., 2001).

\section{*Ceramium fastigiatum Roussel.}

Distribución: Quintana Roo: estuario, insular (Mateo-Cid y Mendoza-González, 1991; Mendoza-González y Mateo-Cid, 1992; Collado-Vides y González-González, 1995).

Nota: Silva et al. (1996) comentan que este nombre se apli- 
ca a una especie de identidad desconocida, por lo que el material debería ser revisado.

Ceramium floridanum J. Agardh.

Distribución: Campeche: litoral (Mateo-Cid et al., 2013b).

Ceramium leptozonum M. Howe.

Distribución: Tabasco: litoral (Ramírez-López, 1996; Sentíes y Dreckmann, 2013; Quiroz-González et al., 2018); Quintana Roo: estuario, litoral, insular (Collado-Vides y González-González, 1995; Ortega et al., 2001; Mateo-Cid et al., 2006).

Ceramium luetzelburgii O.C. Schmidt.

Distribución: Veracruz: estuario, litoral, insular (SánchezRodríguez, 1980; Zizumbo Alamilla, 1995; Mateo-Cid et al., 1996; Ortega et al., 2001; De la Garza-Flores, 2003; NúñezResendiz, 2009; Galicia-García et al., 2013); Tabasco: estuario (Mendoza-González et al., 2017); Campeche: litoral (Mateo-Cid et al., 2013a, b); Quintana Roo: insular (MateoCid y Mendoza-González, 1991; Ortega et al., 2001; MateoCid et al., 2006; Mendoza-González et al., 2007).

Ceramium nitens (C. Agardh) J. Agardh.

Distribución: Veracruz: litoral, insular (Mateo-Cid et al., 1996; Ortega et al., 2001; De la Garza-Flores, 2003; GaliciaGarcía et al., 2013); Campeche: litoral (Ortega et al., 2001; Mateo-Cid et al., 2013a, b; Mendoza-González et al., 2013); Yucatán: litoral, insular (Ortega et al., 2001); Quintana Roo: litoral, insular (Mateo-Cid y Mendoza-González, 1991; Mendoza-González y Mateo-Cid, 1992; Dreckmann et al., 1996; Ortega et al., 2001; Quan-Young et al., 2006).

*Ceramium siliquosum var. zostericola (FeldmannMazoyer) G. Furnari.

ミCeramium diaphanum var. zostericola (Thuret) FeldmannMazoyer.

Distribución: Campeche: estuario (Ortega, 1995; Ortega et al., 2001, en ambos como Ceramium diaphanum var. zostericola).

*Ceramium tenuicorne (Kützing) Waern.

= Ceramium strictum Greville \& Harvey.

Distribución: Quintana Roo: insular (Mateo-Cid y Mendoza-González, 1991; Mendoza-González y Mateo-Cid, 1992, como Ceramium strictum para la región).

Ceramium subtile J. Agardh.

Distribución: Veracruz: litoral, insular (Ortega et al., 2001; De la Garza-Flores, 2003; Godínez-Ortega et al., 2009); Quintana Roo: litoral (Cetz-Navarro et al., 2008).

*Ceramium tenerrimum (G. Martens) Okamura.

Distribución: Quintana Roo: insular (Mateo-Cid y Mendoza-González, 1991; Ortega et al., 2001).

*Ceramium uruguayense W.R. Taylor.

Distribución: Quintana Roo: insular (Ortega et al., 2001).

\section{Ceramium virgatum Roth.}

= Ceramium rubrum C. Agardh.

Distribución: Veracruz: estuario, litoral, insular (Ortega et al., 2001; De la Garza-Flores, 2003, ambos como Ceramium rubrum; Luna-Ortega y De la Cruz-Francisco, 2017); Yucatán: insular (Ortega et al., 2001, como Ceramium rubrum); Quintana Roo: insular (Cetz-Navarro et al., 2008).

Corallophila verongiae (D.L. Ballantine \& M.J. Wynne) R.E. Norris.

Distribución: Quintana Roo: litoral (Cetz-Navarro et al., 2008). 
*Dohrniella antillarum (W.R. Taylor) Feldmann-Mazoyer.

Distribución: Quintana Roo: litoral, insular (Mateo-Cid y Mendoza-González, 1991; Dreckmann et al., 1996; Ortega et al., 2001).

Gayliella fimbriata (Setchell \& N.L. Gardner) T.O. Cho \& S.M. Boo.

Distribución: Tabasco: litoral (Mendoza-González et al., 2017).

Gayliella flaccida (Harvey ex Kützing) T.O. Cho \& L.J. Mclvor. इCeramium flaccidum (Harvey ex Kützing) Ardissone.

Distribución: Tamaulipas: estuario, litoral (Ortega et al., 2001, como Ceramium flaccidum); Veracruz: estuario, litoral, insular (Zizumbo Alamilla, 1995; Mateo-Cid et al., 1996; Ortega et al., 2001; De la Garza-Flores, 2003, reportada como Ceramium flaccidum para esta región; NúñezResendiz, 2009); Tabasco: litoral (Mendoza-González et al., 2017); Campeche: estuario, litoral (Ortega, 1995; Ortega et al., 2001, ambas como Ceramium flaccidum; Mateo-Cid et al., 2013a, b; Mendoza-González et al., 2013); Yucatán: estuario, litoral, insular (Ortega et al., 2001; Ortegón-Aznar et al., 2001, ambas como Ceramium flaccidum); Quintana Roo: estuario, litoral, insular (Mateo-Cid y MendozaGonzález, 1991; Mendoza-González y Mateo-Cid, 1992; Dreckmann et al., 1996; Ortega et al., 2001; Mateo-Cid et al., 2006; Mendoza-González et al., 2007, como Ceramium flaccidum para esta región).

Gayliella mazoyerae T.O. Cho, Fredericq \& Hommersand. = Ceramium gracillimum var. byssoideum Mazoyer.

Distribución: Veracruz: litoral (Sánchez-Rodríguez, 1980, como Ceramium gracillimum var. byssoideum); Tabasco: litoral (Mendoza-González et al., 2017)

Gayliella transversalis (Collins \& Hervey) T.O. Cho \& Fredericq.

= Ceramium byssoideum Harvey.

Distribución: Tamaulipas: estuario, litoral (Vargas López et al., 2004 como Ceramium byssoideum); Tabasco: litoral (Mendoza-González et al., 2017); Campeche: litoral (MateoCid et al., 2013a, b); Quintana Roo: estuario (Collado-Vides y González-González, 1995 como Ceramium byssoideum).

\section{Delesseriaceae}

Apoglossum ruscifolium (Turner) J. Agardh.

Distribución: Campeche: litoral (Mateo-Cid et al., 2013b).

Caloglossa leprieurii (Montagne) G. Martens.

Distribución: Veracruz: estuario, litoral (Ortega et al., 2001; De la Garza-Flores, 2003; De la Cruz-Francisco et al. 2017); Tabasco: litoral (Mendoza-González et al., 2017; QuirozGonzález et al., 2018); Campeche: estuario, litoral (Ortega, 1995; Ortega et al., 2001; Mateo-Cid et al., 2013b); Quintana Roo: litoral, insular (Díaz-Martín y Quan-Young, 2001; Ortega et al., 2001; Quan-Young et al., 2006).

Cottoniella sanguinea $\mathrm{M}$. Howe.

Distribución: Quintana Roo: litoral (Cetz-Navarro et al., 2008).

*Dasya antillarum (M. Howe) A.J.K. Millar. इ Eupogodon antillarum (M. Howe) P.C. Silva.

Distribución: Quintana Roo: litoral (Dreckmann et al., 1996, como Eupogodon antillarum; Ortega et al., 2001).

Dasya baillouviana (S.G. Gmelin) Montagne. = Dasya pedicellata (C. Agardh) C. Agardh .

Distribución: Tamaulipas: litoral (Ortega et al., 2001); Veracruz: litoral, insular (Ortega et al., 2001; De la GarzaFlores, 2003); Tabasco: estuario, litoral (Ramírez-López, 1996; Sentíes y Dreckmann, 2013; Mendoza-González et al., 2017, como Dasya pedicellata; Quiroz-González et al., 2018); Campeche: litoral (Mateo-Cid et al., 2013a); Quintana Roo: litoral, insular (Mateo-Cid y Mendoza-González, 1991; Mendoza-González y Mateo-Cid, 1992; Dreckmann 
et al., 1996; Ortega et al., 2001; Quan-Young et al., 2006; Mateo-Cid et al., 2006; Mendoza-González et al., 2007, como Dasya pedicellata).

\section{Dasya caraibica Børgesen.}

Distribución: Campeche: litoral (Ortega et al., 2001), (Mateo-Cid et al., 2013b); Quintana Roo: litoral, insular (Mendoza-González y Mateo-Cid, 1992; Dreckmann et al., 1996; Ortega et al., 2001; Mateo-Cid et al., 2006; Mendoza-González et al., 2007).

Dasya collinsiana M. Howe.

Distribución: Yucatán: insular (Ortega et al., 2001); Quintana Roo: litoral, insular (Mendoza-González y Mateo-Cid, 1992; Dreckmann et al., 1996; Ortega et al., 2001; MateoCid et al., 2006; Mendoza-González et al., 2007).

Dasya corymbifera J. Agardh.

Distribución: Tamaulipas: litoral (Ortega et al., 2001); Campeche: litoral (Mateo-Cid et al., 2013a, b); Quintana Roo: litoral, insular (Mateo-Cid y Mendoza-González, 1991; Mendoza-González y Mateo-Cid, 1992; Dreckmann et al., 1996; Ortega et al., 2001; Mendoza-González et al., 2007).

Dasya crouaniana J. Agardh.

Distribución: Quintana Roo: litoral, insular (Díaz-Martín y Quan-Young, 2001; Cetz-Navarro et al., 2008).

Dasya haitiana S. Fredericq \& J.N. Norris.

Distribución: Quintana Roo: insular (Mateo-Cid y MendozaGonzález, 1991; Ortega et al., 2001; Mateo-Cid et al., 2006).

Dasya hutchinsiae var. hutchinsiae Harvey.

Distribución: Quintana Roo: insular (Ortega et al., 2001; Mateo-Cid et al., 2006).
Dasya hutchinsiae var. minor (E.C. de Oliveira) M.J. Wynne

Distribución: Campeche: litoral (Mendoza-González et al., 2013); Yucatán: litoral (Ortega et al., 2001).

Dasya mollis Harvey.

Distribución: Quintana Roo: litoral, insular (Dreckmann et al., 1996; Ortega et al., 2001; Mateo-Cid et al., 2006).

Dasya ocellata (Grateloup) Harvey.

Distribución: Veracruz: litoral (Núñez-Resendiz, 2009); Campeche: litoral (Callejas-Jiménez et al., 2005; Mateo-Cid et al., 2013b); Quintana Roo: insular (Mateo-Cid y Mendoza-González, 1991; Ortega et al., 2001; Mateo-Cid et al., 2006).

\section{Dasya ramosissima Harvey.}

Distribución: Quintana Roo: litoral, insular (Mateo-Cid y Mendoza-González, 1991; Collado-Vides y González-González, 1995; Díaz-Martín y Quan-Young, 2001; Ortega et al., 2001; Quan-Young et al., 2006).

Dasya rigidula (Kützing) Ardissone.

Distribución: Tamaulipas: litoral (Ortega et al., 2001); Veracruz: litoral, insular (Ortega et al., 2001; De la Garza-Flores, 2003); Campeche: litoral (Mateo-Cid et al., 2013b; Mendoza-González et al., 2013); Yucatán: litoral (Ortega et al., 2001); Quintana Roo: estuario, litoral, insular (Mateo-Cid y Mendoza-González, 1991; Mendoza-González y Mateo-Cid, 1992; Dreckmann et al., 1996; Ortega et al., 2001; MateoCid et al., 2006; Mendoza-González et al., 2007).

Dictyurus occidentalis J. Agardh.

Distribución: Veracruz: litoral (Ortega et al., 2001; De la Garza-Flores, 2003); Campeche: litoral (Mateo-Cid et al., 2013a); Quintana Roo: insular (Mateo-Cid y Mendoza-González, 1991; Mendoza-González y Mateo-Cid, 1992; Ortega et al., 2001; Mendoza-González et al., 2007). 
Halydictyon mirabile Zanardini.

Distribución: Quintana Roo: litoral, insular (Mateo-Cid et al., 2006; Cetz-Navarro et al., 2008).

Heterosiphonia crispella var. crispella (C. Agardh) M.J. Wynne.

Distribución: Tamaulipas: litoral (Ortega et al., 2001); Tabasco: litoral (Mendoza-González et al., 2017); Campeche: estuario (Ortega, 1995; Ortega et al., 2001; Mateo-Cid et al., 2013a, b; Mendoza-González et al., 2013); Yucatán: estuario, litoral (Ortega et al., 2001; Ortegón-Aznar et al., 2009); Quintana Roo: litoral, insular (Mateo-Cid y Mendoza-González, 1991; Mendoza-González y Mateo-Cid, 1992; Dreckmann et al., 1996; Ortega et al., 2001; Quan-Young et al., 2006).

Heterosiphonia crispella var. Iaxa (Børgesen) M.J. Wynne.

Distribución: Quintana Roo: insular (Mateo-Cid et al., 2006; Mendoza-González et al., 2007).

Heterosiphonia gibbesii (Harvey) Falkenberg.

Distribución: Veracruz: estuario, litoral (Sánchez-Rodríguez, 1980; Ortega et al., 2001; De la Garza-Flores, 2003; NúñezResendiz, 2009); Yucatán: litoral (Ortega et al., 2001); Quintana Roo: litoral, insular (Mateo-Cid y Mendoza-González, 1991; Mendoza-González y Mateo-Cid, 1992; Dreckmann et al., 1996; Ortega et al., 2001; Quan-Young et al., 2006; Mendoza-González et al., 2007).

Hypoglossum hypoglossoides (Stackhouse) Collins \& Hervey.

Distribución: Campeche: litoral (Mateo-Cid et al., 2013a); Quintana Roo: litoral, insular (Mateo-Cid et al., 2006; Mendoza-González et al., 2007; Cetz-Navarro et al., 2008).

Hypoglossum involvens (Harvey) J. Agardh.

Distribución: Quintana Roo: litoral (Cetz-Navarro et al., 2008).
Hypoglossum simulans M.J. Wynne, I.R. Price \& D.L. Ballantine.

Distribución: Veracruz: insular (Godínez-Ortega et al., 2019); Quintana Roo: insular (Mendoza-González y MateoCid, 2007).

Hypoglossum subsimplex M.J. Wynne.

Distribución: Campeche: litoral (Mateo-Cid et al., 2013a); Quintana Roo: insular (Mendoza-González y Mateo-Cid, 2007).

Hypoglossum tenuifolium (Harvey) J. Agardh.

Distribución: Campeche: litoral (Mateo-Cid et al., 2013a); Quintana Roo: litoral, insular (Dreckmann et al., 1996; Ortega et al., 2001; Quan-Young et al., 2006; Mateo-Cid et al., 2006; Vilchis et al., 2018).

Martensia fragilis Harvey.

Distribución: Quintana Roo: litoral, insular (Quan-Young et al., 2006; Mateo-Cid et al., 2006; Cetz-Navarro et al., 2008).

Martensia pavonia (J. Agardh) J. Agardh.

Distribución: Campeche: litoral (Ortega et al., 2001); Yucatán: insular (Ortega et al., 2001); Quintana Roo: insular (Mendoza-González y Mateo-Cid, 1992; Ortega et al., 2001; Mendoza-González et al., 2007).

Nitophyllum adhaerens M.J. Wynne.

Distribución: Campeche: litoral (Mateo-Cid et al., 2013a); Quintana Roo: litoral, insular (Ortega et al., 2001; MateoCid et al., 2006; Mendoza-González et al., 2007).

*Nitophyllum wilkinsoniae Collins \& Hervey.

Distribución: Quintana Roo: insular (Mateo-Cid y Mendoza-González, 1991; Ortega et al., 2001). 
*Platysiphonia caribaea D.L. Ballantine \& M.J. Wynne.

Distribución: Quintana Roo: litoral (Ortega et al., 2001).

Taenioma nanum (Kützing) Papenfuss.

Distribución: Veracruz: litoral, insular (Ortega et al., 2001; De la Garza-Flores, 2003; Godínez-Ortega et al., 2009); Campeche: litoral (Ortega et al., 2001; Mateo-Cid et al., 2013b); Yucatán: insular (Ortega et al., 2001); Quintana Roo: litoral, insular (Mateo-Cid y Mendoza-González, 1991; MendozaGonzález y Mateo-Cid, 1992; Dreckmann et al., 1996; Ortega et al., 2001; Quan-Young et al., 2006; Mateo-Cid et al., 2006).

Taenioma perpusillum (J. Agardh) J. Agardh.

Distribución: Veracruz: litoral (Ortega et al., 2001; De la Garza-Flores, 2003); Campeche: litoral (Ortega et al., 2001; Callejas-Jiménez et al., 2005; Mateo-Cid et al., 2013b); Yucatán: insular (Ortega et al., 2001).

\section{*Thuretia bornetii Vickers.}

Distribución: Quintana Roo: insular (Mateo-Cid y Mendoza-González, 1991; Mendoza-González y Mateo-Cid, 1992; Ortega et al., 2001).

Rhodomelaceae

Acanthophora muscoides (Linnaeus) Bory.

Distribución: Tamaulipas: litoral (Ortega et al., 2001; Vargas López et al., 2004); Veracruz: litoral, insular (Ortega et al., 2001; De la Garza-Flores, 2003); Tabasco: litoral (MendozaGonzález et al., 2017); Campeche: litoral (Mateo-Cid et al., 2013b); Quintana Roo: litoral, insular (Mateo-Cid y Mendoza-González, 1991; Mendoza-González y Mateo-Cid, 1992; Ortega et al., 2001; Quan-Young et al., 2006).

Acanthophora spicifera (M. Vahl) Børgesen.

Distribución: Veracruz: estuario, litoral, insular (Zizumbo Alamilla, 1995; Mateo-Cid et al., 1996; Ortega et al.,
2001; De la Garza-Flores, 2003; González-Gándara et al., 2007; Núñez-Resendiz, 2009; De la Cruz-Francisco et al. 2017; Vilchis et al. 2018; Landa-Cansigno et al., 2019); Tabasco: estuario, litoral (Ramírez-López, 1996; Sentíes y Dreckmann, 2013; Mendoza-González et al., 2017; Vilchis et al. 2018; Quiroz-González et al., 2018); Campeche: estuario, litoral (Ortega, 1995; Ortega et al., 2001; CallejasJiménez et al., 2005; Mateo-Cid et al., 2013b; Vilchis et al. 2018; Núñez-Resendiz et al., 2019b); Yucatán: estuario, litoral, insular (Ortega et al., 2001; Ortegón-Aznar et al., 2001, 2009; Vilchis et al. 2018; Núñez-Resendiz et al., 2019a); Quintana Roo: estuario, litoral, insular (MateoCid y Mendoza-González, 1991; Mendoza-González y Mateo-Cid, 1992; Collado-Vides y González-González, 1995; Dreckmann et al., 1996; Ortega et al., 2001; Mateo-Cid et al., 2006; Mendoza-González et al., 2007; Núñez-Resendiz et al., 2019a).

Acanthosiphonia echinata (Harvey) A.M. Savoie \& G.W. Saunders. ミPolysiphonia echinata Harvey.

= Polysiphonia fracta Harvey.

Distribución: Veracruz: estuario, litoral (Ortega et al., 2001; De la Garza-Flores, 2003, ambas como Polysiphonia echinata); Quintana Roo: estuario, insular (Mateo-Cid y Mendoza-González, 1991; Collado-Vides y González-González, 1995; Ortega et al., 2001, reportada como Polysiphonia fracta para esta región).

Alsidium seaforthii (Turner) J. Agardh. 三Bryothamnion seaforthii (Turner) Kützing.

Distribución: Tamaulipas: litoral (Vargas López et al., 2004); Veracruz: estuario, litoral, insular (Sánchez-Rodríguez, 1980; Ortega et al., 2001; De la Garza-Flores, 2003; NúñezResendiz, 2009; De la Cruz-Francisco et al. 2017); Campeche: litoral (Ortega et al., 2001; Mateo-Cid et al., 2013a, b; Mendoza-González et al., 2013; Núñez-Resendiz et al., 2019b); Yucatán: estuario, litoral (Ortega et al., 2001; Ortegón-Aznar et al., 2001; Núñez-Resendiz et al., 2019b); Quintana Roo: litoral, insular (Mendoza-González y Mateo-Cid, 1992; Dreckmann et al., 1996; Ortega et al., 2001; Núñez- 
Resendiz et al., 2019b). Reportada como Bryothamnion seaforthii para todas las regiones.

Nota: esta especie había sido ampliamente registrada en el AM como Bryothamnion seaforthii; sin embargo, a partir de evidencia molecular, las especies del género Bryothamnion Kützing fueron transferidas al género Alsidium C. Agardh (García-Soto y López-Bautista, 2018).

Alsidium triquetrum (S.G. Gmelin) Trevisan. 三 Bryothamnion triquetrum (S.G. Gmelin) M. Howe.

Distribución: Tamaulipas: litoral (Ortega et al., 2001; Vargas López et al., 2004); Veracruz: estuario, litoral, insular (Sánchez-Rodríguez, 1980; Ortega et al., 2001; De la GarzaFlores, 2003; Núñez-Resendiz, 2009; Galicia-García et al., 2013; De la Cruz-Francisco et al. 2017; Landa-Cansigno et al., 2019); Campeche: litoral (Callejas-Jiménez et al., 2005; Mateo-Cid et al., 2013a, b; Núñez-Resendiz et al., 2019b); Yucatán: estuario, litoral (Ortega et al., 2001; Ortegón-Aznar et al., 2009; Núñez-Resendiz et al., 2019b); Quintana Roo: litoral, insular (Mateo-Cid y Mendoza-González, 1991; Mendoza-González y Mateo-Cid, 1992; Dreckmann et al., 1996; Ortega et al., 2001; Mendoza-González et al., 2007).

Nota: esta especie había sido ampliamente registrada en el AM como Bryothamnion seaforthii; sin embargo, a partir de evidencia molecular, las especies del género Bryothamnion fueron transferidas al género Alsidium (García-Soto y López-Bautista, 2018).

Amansia multifida J.V. Lamouroux.

Distribución: Veracruz: litoral (Ortega et al., 2001; De la Garza-Flores, 2003; Núñez-Resendiz, 2009; Vilchis et al., 2018); Quintana Roo: litoral, insular (Mendoza-González y Mateo-Cid, 1992; Ortega et al., 2001; Vilchis et al., 2018).

\section{Bostrychia binderi Harvey.}

Distribución: Veracruz: litoral (De la Garza-Flores, 2003); Quintana Roo: insular (Mateo-Cid y Mendoza-González, 1991; Mendoza-González y Mateo-Cid, 1992).
Bostrychia calliptera (Montagne) Montagne.

= Bostrychia pinnata J. Tanaka \& Chihara.

Distribución: Veracruz: estuario, litoral (Ortega et al., 2001; De la Garza-Flores, 2003, ambas como Bostrychia pinnata); Yucatán: estuario (Ortegón-Aznar et al., 2001).

Bostrychia montagnei Harvey.

Distribución: Yucatán: estuario (Ortegón-Aznar et al., 2001); Quintana Roo: litoral, insular (Ortega et al., 2001).

Bostrychia moritziana (Sonder ex Kützing) J. Agardh.

Distribución: Veracruz: estuario, litoral (Ortega et al., 2001; De la Garza-Flores, 2003); Yucatán: estuario (Ortegón-Aznar et al., 2001); Quintana Roo: litoral (Cetz-Navarro et al., 2008).

\section{Bostrychia pilulifera Montagne.}

Distribución: Quintana Roo: litoral (Cetz-Navarro et al., 2008).

Bostrychia radicans (Montagne) Montagne.

Distribución: Veracruz: estuario, litoral (Sánchez-Rodríguez, 1980; Ortega et al., 2001; De la Garza-Flores, 2003); Tabasco: litoral (Mendoza-González et al., 2017); Campeche: estuario (Ortega, 1995; Ortega et al., 2001; Mateo-Cid et al., 2013b); Yucatán: estuario (Ortegón-Aznar et al., 2001); Quintana Roo: litoral (Ortega et al., 2001).

Bostrychia scorpioides (Hudson) Montagne.

Distribución: Veracruz: litoral (De la Garza-Flores, 2003); Quintana Roo: estuario, litoral, insular (Ortega et al., 2001).

Bostrychia tenella (J.V. Lamouroux) J. Agardh.

Distribución: Veracruz: litoral (Ortega et al., 2001; De la Garza-Flores, 2003); Campeche: estuario, litoral (Ortega et 
al., 2001; Mateo-Cid et al., 2013b); Yucatán: estuario, litoral (Ortega et al., 2001; Ortegón-Aznar et al., 2001, 2009); Quintana Roo: litoral, insular (Mateo-Cid y Mendoza-González, 1991; Mendoza-González y Mateo-Cid, 1992; Ortega et al., 2001).

Bryocladia cuspidata (J. Agardh) De Toni.

Distribución: Tamaulipas: litoral (Ortega et al., 2001; Vargas López et al., 2004); Veracruz: litoral, insular (Sánchez-Rodríguez, 1980; Ortega et al., 2001; De la Garza-Flores, 2003; Núñez-Resendiz, 2009); Tabasco: estuario, litoral (RamírezLópez, 1996; Sentíes y Dreckmann, 2013; Mendoza-González et al., 2017; Quiroz-González et al., 2018); Campeche: litoral (Ortega et al., 2001; Callejas-Jiménez et al., 2005; Mateo-Cid et al., 2013b); Yucatán: insular (Ortega et al., 2001); Quintana Roo: insular (Díaz-Martín y Quan-Young, 2001; Ortega et al., 2001; Quan-Young et al., 2006).

Bryocladia thyrsigera (J. Agardh) F. Schmitz.

Distribución: Tamaulipas: litoral (Ortega et al., 2001; Vargas López et al., 2004); Tabasco: estuario, litoral (MendozaGonzález et al., 2017; Quiroz-González et al., 2018).

Carradoriella denudata (Dillwyn) A.M. Savoie \& G.W. Saunders.

ミPolysiphonia denudata (Dillwyn) Greville ex Harvey.

Distribución: Tamaulipas: estuario, litoral (Ortega et al., 2001); Tabasco: litoral (Quiroz-González et al., 2018); Campeche: litoral (Mateo-Cid et al., 2013b; Mendoza-González et al., 2013); Quintana Roo: insular (Mateo-Cid y MendozaGonzález, 1991; Mendoza-González y Mateo-Cid, 1992; Ortega et al., 2001).

Nota: reportada como Polysiphonia denudata para estas regiones.

Chondria atropurpurea Harvey.

Distribución: Tamaulipas: litoral (Vargas López et al.,
2004); Veracruz: litoral (De la Cruz-Francisco et al. 2017); Yucatán: litoral (Ortega et al., 2001); Quintana Roo: litoral, insular (Mendoza-González y Mateo-Cid, 1992; Dreckmann et al., 1996; Ortega et al., 2001; Quan-Young et al., 2006).

Chondria baileyana (Montagne) Harvey.

Distribución: Campeche: estuario (Ortega, 1995; Ortega et al., 2001); Quintana Roo: estuario, litoral, insular (MateoCid y Mendoza-González, 1991; Mendoza-González y Mateo-Cid, 1992; Collado-Vides y González-González, 1995; Dreckmann et al., 1996; Ortega et al., 2001; Quan-Young et al., 2006).

Chondria capillaris (Hudson) M.J. Wynne.

= Chondria tenuissima (Withering) C. Agardh.

Distribución: Tamaulipas: litoral (Ortega et al., 2001); Veracruz: estuario, litoral (Ortega et al., 2001; De la Garza-Flores, 2003); Campeche: estuario, litoral (Ortega, 1995, como Chondria tenuissima; Ortega et al., 2001; Callejas-Jiménez et al., 2005; Mateo-Cid et al., 2013b); Yucatán: litoral (Ortegón-Aznar et al., 2009); Quintana Roo: estuario, litoral, insular (Mateo-Cid y MendozaGonzález, 1991; Mendoza-González y Mateo-Cid, 1992; Dreckmann et al., 1996, reportada como Chondria tenuissima para esta región; Ortega et al., 2001; QuanYoung et al., 2006).

Chondria cnicophylla (Melvill) De Toni.

Distribución: Tamaulipas: litoral (Ortega et al., 2001; Vargas López et al., 2004); Campeche: litoral (Mateo-Cid et al., 2013b); Quintana Roo: litoral, insular (Dreckmann et al., 1996; Ortega et al., 2001).

Chondria collinsiana M. Howe.

Distribución: Tabasco: litoral (Mendoza-González et al., 2017); Campeche: litoral (Mateo-Cid et al., 2013b); Quintana Roo: estuario, insular (Collado-Vides y González-González, 1995; Ortega et al., 2001). 
Chondria curvilineata Collins \& Hervey.

Distribución: Tamaulipas: estuario (Ortega et al., 2001); Veracruz: litoral, insular (Mateo-Cid et al., 1996; Ortega et al., 2001; De la Garza-Flores, 2003); Campeche: litoral (Mateo-Cid et al., 2013b); Yucatán: insular (Ortega et al., 2001); Quintana Roo: litoral, insular (Mateo-Cid y Mendoza-González, 1991; Mendoza-González y Mateo-Cid, 1992; Dreckmann et al., 1996; Ortega et al., 2001; Mateo-Cid et al., 2006).

Chondria dasyphylla (Woodward) C. Agardh.

Distribución: Tamaulipas: litoral (Ortega et al., 2001; Vargas López et al., 2004); Veracruz: estuario, litoral (Ortega et al., 2001; De la Garza-Flores, 2003); Campeche: litoral (Mateo-Cid et al., 2013b); Yucatán: estuario, insular (Ortega et al., 2001; Ortegón-Aznar et al., 2001, 2009); Quintana Roo: litoral, insular (Mateo-Cid y Mendoza-González, 1991; Mendoza-González y Mateo-Cid, 1992; Ortega et al., 2001; Mateo-Cid et al., 2006; Mendoza-González et al., 2007).

Chondria floridana (Collins) M. Howe.

Distribución: Yucatán: insular (Ortega et al., 2001); Quintana Roo: insular (Díaz-Martín y Quan-Young, 2001; Ortega et al., 2001; Mendoza-González et al., 2007).

Chondria leptacremon (Melvill ex G. Murray) De Toni.

Distribución: Veracruz: litoral, insular (Ortega et al., 2001; De la Garza-Flores, 2003); Tabasco: litoral (Ramírez-López, 1996; Sentíes y Dreckmann, 2013; Quiroz-González et al., 2018); Quintana Roo: insular (Ortega et al., 2001).

Chondria littoralis Harvey.

Distribución: Veracruz: litoral, insular (Ortega et al., 2001; De la Garza-Flores, 2003; González-Gándara et al., 2007; Godínez-Ortega et al., 2009); Tabasco: estuario, litoral (Ortega et al., 2001; Sentíes y Dreckmann, 2013; Quiroz-González et al., 2018); Campeche: litoral (Callejas-Jiménez et al., 2005;
Mateo-Cid et al., 2013b); Yucatán: estuario, insular (Ortega et al., 2001; Ortegón-Aznar et al., 2009); Quintana Roo: estuario, litoral, insular (Mateo-Cid y Mendoza-González, 1991; Mendoza-González y Mateo-Cid, 1992; Collado-Vides y González-González, 1995; Dreckmann et al., 1996; Ortega et al., 2001).

Chondria platyramea A.B. Joly \& Ugadim.

Distribución: Quintana Roo: litoral, insular (Dreckmann et al., 1996; Ortega et al., 2001; Mateo-Cid et al., 2006; Mendoza-González et al., 2007).

Chondria polyrhiza Collins \& Hervey.

Distribución: Veracruz: litoral, insular (Ortega et al., 2001; De la Garza-Flores, 2003; Godínez-Ortega et al., 2009); Tabasco: estuario, litoral (Ortega et al., 2001; Sentíes y Dreckmann, 2013; Quiroz-González et al., 2018); Campeche: litoral (Mateo-Cid et al., 2013a, b); Yucatán: litoral, insular (Ortega et al., 2001; Ortegón-Aznar et al., 2009); Quintana Roo: litoral, insular (Mateo-Cid y Mendoza-González, 1991; Dreckmann et al., 1996; Ortega et al., 2001; Mateo-Cid et al., 2006; Mendoza-González et al., 2007).

\section{Chondria sedifolia Harvey.}

Distribución: Veracruz: litoral, insular (Ortega et al., 2001; De la Garza-Flores, 2003; Godínez-Ortega et al., 2009); Campeche: estuario (Ortega, 1995; Ortega et al., 2001; Mateo-Cid et al., 2013b); Yucatán: insular (Ortega et al., 2001); Quintana Roo: litoral, insular (Ortega et al., 2001).

Chondrophycus anabeliae Sentíes, M.T. Fujii, Cassano \& Dreckmann.

Distribución: Quintana Roo: insular (Sentíes et al., 2016).

Digenea mexicana G.H. Boo \& D. Robledo.

Distribución: Tamaulipas: litoral (Dreckmann y Sentíes, 1994; Ortega et al., 2001; Vargas López et al., 2004; Vilchis 
et al., 2018); Veracruz: estuario, litoral, insular (SánchezRodríguez, 1980; Dreckmann y Sentíes, 1994; Zizumbo Alamilla, 1995; Mateo-Cid et al., 1996; Ortega et al., 2001; De la Garza-Flores, 2003; Núñez-Resendiz, 2009; De la Cruz-Francisco et al. 2017; Boo et al., 2018; Vilchis et al., 2018; Landa-Cansigno et al., 2019); Campeche: estuario, litoral (Dreckmann y Sentíes, 1994; Ortega, 1995; Ortega et al., 2001; Callejas-Jiménez et al., 2005; Mateo-Cid et al., 2013b; Mendoza-González et al., 2013; Vilchis et al., 2018; Núñez-Resendiz et al., 2019b); Yucatán: litoral, insular (Dreckmann y Sentíes, 1994; Ortega et al., 2001; OrtegónAznar et al., 2009; Vilchis et al., 2018; Núñez-Resendiz et al., 2019b); Quintana Roo: estuario, litoral, insular (MateoCid y Mendoza-González, 1991; Mendoza-González y Mateo-Cid, 1992; Dreckmann y Sentíes, 1994; Collado-Vides y González-González, 1995; Dreckmann et al., 1996; Ortega et al., 2001; Quan-Young et al., 2006; Mateo-Cid et al., 2006; Boo et al., 2018; Vilchis et al., 2018; Núñez-Resendiz et al., 2019b).

Nota: esta especie ha sido registrada en toda la región del AM como Digenea simplex (a partir de Sánchez-Rodríguez, 1980; Ortega et al., 2001; hasta Núñez-Resendiz et al., 2019b). Sin embargo, de acuerdo con Schneider et al. (2018), a partir de evidencia molecular, la distribución de esta especie aparentemente pantropical está ahora restringida a la región del Mediterráneo. La única especie de Digenea $\mathrm{C}$. Agardh que hasta ahora se encuentra registrada para la Península de Yucatán es D. mexicana (Boo et al., 2018).

*Dipterosiphonia dendritica (C. Agardh) F. Schmitz.

Distribución: Quintana Roo: litoral, insular (Mateo-Cid y Mendoza-González, 1991; Dreckmann et al., 1996; Ortega et al., 2001).

*Dipterosiphonia rigens (C. Agardh) Falkenberg.

Distribución: Yucatán: insular (Ortega et al., 2001); Quintana Roo: litoral, insular (Mateo-Cid y Mendoza-González, 1991; Ortega et al., 2001).
Herposiphonia bipinnata M. Howe.

Distribución: Quintana Roo: insular (Ortega et al., 2001; Mateo-Cid et al., 2006).

Herposiphonia pecten-veneris var. pecten-veneris (Harvey) Falkenberg.

Distribución: Veracruz: litoral (Ortega et al., 2001; De la Garza-Flores, 2003; Godínez-Ortega et al., 2009); Campeche: litoral (Ortega et al., 2001); Yucatán: insular (Ortega et al., 2001); Quintana Roo: estuario, litoral, insular (MateoCid y Mendoza-González, 1991; Collado-Vides y GonzálezGonzález, 1995; Dreckmann et al., 1996; Ortega et al., 2001; Quan-Young et al., 2006).

Herposiphonia pecten-veneris var. laxa W.R. Taylor.

Distribución: Veracruz: litoral, insular (Ortega et al., 2001; De la Garza-Flores, 2003); Quintana Roo: estuario, insular (Collado-Vides y González-González, 1995; Ortega et al., 2001; Quan-Young et al., 2006).

Herposiphonia secunda f. secunda (C. Agardh) Ambronn.

Distribución: Tamaulipas: estuario, litoral (Ortega et al., 2001); Veracruz: estuario, litoral, insular (Ortega et al., 2001; De la Garza-Flores, 2003); Campeche: estuario, litoral (Ortega et al., 2001); Yucatán: litoral, insular (Ortega et al., 2001; Ortegón-Aznar et al., 2001, 2009); Quintana Roo: estuario, litoral, insular (Mateo-Cid y Mendoza-González, 1991; Mendoza-González y Mateo-Cid, 1992; Collado-Vides y González-González, 1995; Dreckmann et al., 1996; Ortega et al., 2001; Quan-Young et al., 2006; Mendoza-González et al., 2007).

Herposiphonia secunda f. tenella (C. Agardh) M.J. Wynne.

Distribución: Veracruz: estuario, litoral (Ortega et al., 2001; De la Garza-Flores, 2003); Campeche: estuario, litoral (Ortega, 1995; Ortega et al., 2001; Mateo-Cid et al., 2013b); Yucatán: litoral, insular (Ortega et al., 2001; Ortegón-Aznar 
et al., 2001); Quintana Roo: estuario, litoral, insular (Mateo-Cid y Mendoza-González, 1991; Mendoza-González y Mateo-Cid, 1992; Dreckmann et al., 1996; Ortega et al., 2001; Mateo-Cid et al., 2006).

Herposiphonia tenella (C. Agardh) Ambronn.

Distribución: Veracruz: litoral (Sánchez-Rodríguez, 1980; De la Garza-Flores, 2003).

Heterodasya mucronata (Harvey) M.J. Wynne. 三 Micropeuce mucronata (Harvey) Kylin ex E.C. Oliveira.

Distribución: Tamaulipas: litoral (Ortega et al., 2001); Yucatán: litoral (Ortega et al., 2001); Quintana Roo: litoral, insular (Mateo-Cid y Mendoza-González, 1991; MendozaGonzález y Mateo-Cid, 1992; Dreckmann et al., 1996; Ortega et al., 2001; Mateo-Cid et al., 2006; Mendoza-González et al., 2007).

Nota: reportada como Micropeuce mucronata para estas regiones.

Kapraunia schneideri (Stuercke \& Freshwater) A.M. Savoie \& G.W. Saunders.

三 Polysiphonia schneideri B. Stuercke \& D.W. Freshwater.

Distribución: Tabasco: litoral (Mendoza-González et al., 2017, como Polysiphonia schneideri).

Laurencia brongniartii J. Agardh.

Distribución: Quintana Roo: insular (Mendoza-González y Mateo-Cid, 1992; Ortega et al., 2001; Sentíes y Fujii, 2002; Mendoza-González et al., 2007; Hernández et al., 2017).

Laurencia caraibica P.C. Silva.

Distribución: Tamaulipas: litoral (Ortega et al., 2001; Hernández et al., 2017); Veracruz: litoral, insular (Ortega et al., 2001; De la Garza-Flores, 2003; Hernández et al., 2017); Campeche: estuario (Ortega et al., 2001; Mateo-Cid et al., 2013a; Hernández et al., 2017); Quintana Roo: litoral, insu- lar (Mateo-Cid y Mendoza-González, 1991; Mendoza-González y Mateo-Cid, 1992; Dreckmann et al., 1996; Ortega et al., 2001; Sentíes y Fujii, 2002; Mendoza-González et al., 2007; Cassano et al., 2012; Sentíes et al., 2015; Hernández et al., 2017).

Laurencia chondrioides Børgesen.

Distribución: Quintana Roo: litoral (Ortega et al., 2001; Sentíes y Fujii, 2002; Hernández et al., 2017).

Laurencia corymbosa J. Agardh.

Distribución: Campeche: litoral (Ortega et al., 2001); Yucatán: insular (Ortega et al., 2001); Quintana Roo: insular (Ortega et al., 2001).

Nota: para el Caribe mexicano se consideró como un registro dudoso en Sentíes y Fujii (2002); hasta la fecha no se ha tenido otro registro de la especie.

Laurencia filiformis (C. Agardh) Montagne.

Distribución: Quintana Roo: litoral, insular (Mateo-Cid y Mendoza-González, 1991; Mendoza-González y Mateo-Cid, 1992; Dreckmann et al., 1996; Ortega et al., 2001; Sentíes y Fujii, 2002; Vilchis et al., 2018).

*Laurencia hancockii E.Y. Dawson.

Distribución: Quintana Roo: insular (Ortega et al., 2001).

Laurencia intricata J.V. Lamouroux.

Distribución: Veracruz: litoral, insular (Ortega et al., 2001; De la Garza-Flores, 2003; González-Gándara et al., 2007; Galicia-García et al., 2013); Campeche: litoral (Ortega et al., 2001; Callejas-Jiménez et al., 2005; Cassano et al., 2012; Mateo-Cid et al., 2013a, b; Mendoza-González et al., 2013; Hernández et al., 2017); Yucatán: estuario, litoral, insular (Ortega et al., 2001; Ortegón-Aznar et al., 2009; Núñez-Resendiz et al., 2019b); Quintana Roo: estuario, litoral, insular (Mendoza-González y Mateo-Cid, 
1992; Dreckmann et al., 1996; Ortega et al., 2001; Sentíes y Fujii, 2002; Quan-Young et al., 2006; Mateo-Cid et al., 2006; Hernández et al., 2017; Núñez-Resendiz et al., 2019b).

Laurencia laurahuertana Mateo-Cid, Mendoza-González, Sentíes \& Díaz-Larrea.

Distribución: Quintana Roo: litoral (Mateo-Cid et al., 2014b; Hernández et al., 2017).

\section{Laurencia microcladia Kützing.}

Distribución: Tamaulipas: litoral (Ortega et al., 2001); Veracruz: estuario, litoral, insular (Sánchez-Rodríguez, 1980; Ortega et al., 2001; De la Garza-Flores, 2003; Núñez-Resendiz, 2009); Campeche: estuario, litoral (Ortega et al., 2001; Mateo-Cid et al., 2013b); Yucatán: estuario, litoral (Ortega et al., 2001; Ortegón-Aznar et al., 2009); Quintana Roo: estuario, litoral, insular (Mateo-Cid y MendozaGonzález, 1991; Mendoza-González y Mateo-Cid, 1992; Collado-Vides y González-González, 1995; Dreckmann et al., 1996; Ortega et al., 2001; Sentíes y Fujii, 2002; QuanYoung et al., 2006).

Laurencia obtusa (Hudson) J.V. Lamouroux.

Distribución: Tamaulipas: litoral (Ortega et al., 2001; Vargas López et al., 2004; Hernández et al., 2017); Veracruz: estuario, litoral, insular (Sánchez-Rodríguez, 1980; Zizumbo Alamilla, 1995; Mateo-Cid et al., 1996; Ortega et al., 2001; De la Garza-Flores, 2003; Godínez-Ortega et al., 2009; Núñez-Resendiz, 2009; Galicia-García et al., 2013; Hernández et al., 2017; Luna-Ortega y De la Cruz-Francisco, 2017; Landa-Cansigno et al., 2019); Campeche: litoral (Ortega et al., 2001; Mateo-Cid et al., 2013a; Mendoza-González et al., 2013; Hernández et al., 2017); Yucatán: litoral, insular (Ortega et al., 2001; Hernández et al., 2017); Quintana Roo: litoral, insular (Mateo-Cid y Mendoza-González, 1991; Mendoza-González y Mateo-Cid, 1992; Dreckmann et al., 1996; Ortega et al., 2001; Sentíes y Fujii, 2002; Quan-Young et al., 2006; Mateo-Cid et al., 2006; Mendoza-González et al., 2007; Hernández et al., 2017).
Nota: Laurencia obtusa ha sido reconocida como una especie de amplia distribución. Cassano et al. (2012) consideran que los registros de esta especie para Brasil corresponden a L. dendroidea J. Agardh. Análisis moleculares posteriores podrían confirmar esta suposición.

\section{Laurencia venusta Yamada.}

Distribución: Quintana Roo: litoral (Sentíes et al., 2001; Sentíes y Fujii, 2002; Díaz-Larrea et al., 2007; Cassano et al., 2012; Sentíes et al., 2015; Hernández et al., 2017).

Laurenciella marilzae (Gil-Rodríguez, Sentíes, Díaz-Larrea, Cassano \& M.T. Fujii) Gil-Rodríguez, Sentíes, Díaz-Larrea, Cassano \& M.T. Fujii.

三 Laurencia marilzae Gil-Rodríguez, Sentíes, Díaz-Larrea, Cassano \& M.T. Fujii.

Distribución: Quintana Roo: insular (Sentíes et al., 2011, como Laurencia marilzae; Cassano et al., 2012; Sentíes et al., 2015).

Lophocladia trichoclados (C. Agardh) F. Schmitz.

Distribución: Tamaulipas: litoral (Ortega et al., 2001); Campeche: estuario (Ortega, 1995; Ortega et al., 2001; MateoCid et al., 2013a, b); Quintana Roo: litoral, insular (Dreckmann et al., 1996; Ortega et al., 2001; Mateo-Cid et al., 2006).

Lophosiphonia cristata Falkenberg.

Distribución: Campeche: litoral (Mateo-Cid et al., 2013b); Yucatán: estuario, insular (Ortega et al., 2001; Ortegón-Aznar et al., 2001); Quintana Roo: litoral, insular (Dreckmann et al., 1996; Ortega et al., 2001; Mendoza-González et al., 2007).

Lophosiphonia obscura (C. Agardh) Falkenberg.

Distribución: Campeche: litoral (Mateo-Cid et al., 2013b); Yucatán: insular (Ortega et al., 2001); Quintana Roo: litoral (Cetz-Navarro et al., 2008). 
*Melanothamnus eastwoodiae (Setchell \& N.L. Gardner) Díaz-Tapia \& Maggs.

इ Polysiphonia eastwoodiae Setchell \& N.L. Gardner.

Distribución: Quintana Roo: estuario (Collado-Vides y González-González, 1995, como Polysiphonia eastwoodiae).

Melanothamnus ferulaceus (Suhr ex J. Agardh) Díaz-Tapia \& Maggs.

इ Polysiphonia ferulacea Suhr ex J.Agardh.

इ Neosiphonia ferulacea (Suhr ex J.Agardh) S.M. Guimarães \& M.T. Fujii.

Distribución: Tamaulipas: estuario, litoral (Ortega et al., 2001; Vargas López et al., 2004, como Polysiphonia ferulacea para la región); Veracruz: insular (SánchezRodríguez, 1980; Ortega et al., 2001; De la Garza-Flores, 2003, como Polysiphonia ferulacea en las tres citas anteriores; Godínez-Ortega et al., 2009, como Neosiphonia ferulacea); Tabasco: estuario, litoral (Ortega et al., 2001, como Polysiphonia ferulacea; Sentíes y Dreckmann, 2013, como Neosiphonia ferulacea; Mendoza-González et al., 2017; Quiroz-González et al., 2018); Campeche: estuario (Ortega, 1995; Ortega et al., 2001, como Polysiphonia ferulacea para esta región); Yucatán: litoral (Ortegón-Aznar et al., 2009, como Neosiphonia ferulacea); Quintana Roo: insular (Mateo-Cid y Mendoza-González, 1991; MendozaGonzález y Mateo-Cid, 1992; Dreckmann et al., 1996; Ortega et al., 2001, como Polysiphonia ferulacea en las cuatro referencias anteriores; Mateo-Cid et al., 2006, como Neosiphonia ferulacea; Mendoza-González et al., 2007).

Melanothamnus gorgoniae (Harvey) Díaz-Tapia \& Maggs. 三 Polysiphonia gorgoniae Harvey.

三 Neosiphonia gorgoniae (Harvey) S.M. Guimarães \& M.T. Fujii.

Distribución: Veracruz: litoral, insular (Zizumbo Alamilla, 1995; Ortega et al., 2001, ambas como Polysiphonia gorgoniae; De la Garza-Flores, 2003; Galicia-García et al., 2013, ambas como Neosiphonia gorgoniae); Campeche: estuario, litoral (Ortega et al., 2001, como Polysiphonia gorgoniae; Mendoza-González et al., 2013, como Neosiphonia gorgoniae); Yucatán: insular (Ortega et al., 2001); Quintana Roo: estuario, litoral, insular (Mateo-Cid y Mendoza-González, 1991, como Polysiphonia gorgoniae; Mendoza-González y Mateo-Cid, 1992; Collado-Vides y González-González, 1995; Dreckmann et al., 1996; Ortega et al., 2001, como Polysiphonia gorgoniae; Mateo-Cid et al., 2006).

Melanothamnus pseudovillum (Hollenberg) Díaz-Tapia \& Maggs.

इ Polysiphonia pseudovillum Hollenberg.

Distribución: Veracruz: litoral (Galicia-García et al., 2013, como Polysiphonia pseudovillum).

Melanothamnus sphaerocarpus (Børgesen) Díaz-Tapia \& Maggs.

ミPolysiphonia sphaerocarpa Børgesen.

इ Neosiphonia sphaerocarpa (Børgesen) M.S. Kim \& I.K. Lee.

Distribución: Veracruz: estuario, litoral, insular (Mateo-Cid et al., 1996; Ortega et al., 2001, ambas como Polysiphonia sphaerocarpa; De la Garza-Flores, 2003; Núñez-Resendiz, 2009, ambas como Neosiphonia sphaerocarpa); Tabasco: estuario, litoral (Ramírez-López, 1996; Ortega et al., 2001, ambas como Polysiphonia sphaerocarpa; Sentíes y Dreckmann, 2013, como Neosiphonia sphaerocarpa; Mendoza-González et al., 2017; Quiroz-González et al., 2018); Campeche: litoral (Mateo-Cid et al., 2013b; Mendoza-González et al., 2013, ambas como Neosiphonia sphaerocarpa); Yucatán: estuario, litoral, insular (Ortega et al., 2001; Ortegón-Aznar et al., 2001, ambas como Polysiphonia sphaerocarpa, Núñez-Resendiz, 2009, como Neosiphonia sphaerocarpa); Quintana Roo: estuario, insular (MateoCid y Mendoza-González, 1991; Mendoza-González y Mateo-Cid, 1992; Collado-Vides y González-González, 1995; Dreckmann et al., 1996; Ortega et al., 2001; Quan-Young et al., 2006; Mateo-Cid et al., 2006, ambas como Neosiphonia sphaerocarpa). 
*Melanothamnus tongatensis (Harvey ex Kützing) DíazTapia \& Maggs.

三 Neosiphonia tongatensis (Harvey ex Kützing) M.S. Kim \& I.K. Lee.

Distribución: Quintana Roo: estuario, litoral (Ortega et al., 2001, como Neosiphonia tongatensis).

Micropeuce sarcocaulon (Harvey) Kylin ex P.C. Silva.

Distribución: Quintana Roo: litoral (Cetz-Navarro et al., 2008).

Murrayella periclados (C. Agardh) F. Schmitz.

Distribución: Campeche: litoral (Mateo-Cid et al., 2013b); Yucatán: estuario, litoral (Ortegón-Aznar et al., 2001, 2009); Quintana Roo: estuario, litoral, insular (Mateo-Cid y Mendoza-González, 1991; Mendoza-González y Mateo-Cid, 1992; Ortega et al., 2001).

Ohelopapa flexilis (Setchell) F. Rousseau, Martin-Lescanne, Payri \& L. Le Gall.

$\equiv$ Laurencia flexilis Setchell.

Distribución: Quintana Roo: insular (Ortega et al., 2001, como Laurencia flexilis).

Ophidocladus simpliciusculus (P. Crouan \& H. Crouan) Falkenberg.

Distribución: Veracruz: litoral, insular (Ortega et al., 2001; De la Garza-Flores, 2003).

Osmundaria obtusiloba (C. Agardh) R.E. Norris. EVidalia obtusiloba (Mertens ex C. Agardh) J. Agardh.

Distribución: Veracruz: litoral (Sánchez-Rodríguez, 1980, como Vidalia obtusiloba Ortega et al., 2001; De la GarzaFlores, 2003; Núñez-Resendiz, 2009); Campeche: litoral (Mendoza-González et al., 2013); Quintana Roo: insular (Mendoza-González y Mateo-Cid, 1992; Ortega et al., 2001; Mendoza-González et al., 2007).
Osmundea lata (M. Howe \& W.R. Taylor) Y. Yoneshigue-Valentin, M.T. Fujii \& C.F. Gurgel.

Distribución: Campeche: litoral (Mateo-Cid et al., 2013a).

Palisada corallopsis (Montagne) Sentíes, M.T. Fujii \& DíazLarrea.

इ Laurencia corallopsis (Montagne) M. Howe.

三Chondrophycus corallopsis (Montagne) K.W. Nam.

Distribución: Veracruz: litoral, insular (Ortega et al., 2001; De la Garza-Flores, 2003, ambas como Laurencia corallopsis); Campeche: litoral (Mateo-Cid et al., 2013b; MendozaGonzález et al., 2013); Yucatán: insular (Ortega et al., 2001, como Laurencia corallopsis); Quintana Roo: insular (MateoCid y Mendoza-González, 1991; Mendoza-González y MateoCid, 1992; Ortega et al., 2001, reportada como Laurencia corallopsis para esta región; Díaz-Larrea et al., 2007, como Chondrophycus corallopsis; Cassano et al., 2012).

Palisada flagellifera (J. Agardh) K.W. Nam.

इ Laurencia flagellifera J. Agardh.

Distribución: Quintana Roo: estuario, litoral, insular (Mateo-Cid y Mendoza-González, 1991; Mendoza-González y Mateo-Cid, 1992; Dreckmann et al., 1996; Ortega et al., 2001; Sentíes y Fujii, 2002, reportada como Laurencia flagellifera en las cinco referencias anteriores; Vilchis et al., 2018).

Palisada perforata (Bory) K.W. Nam.

= Chondrophycus papillosus (C. Agardh) D.J. Garbary \& J.T. Harper.

= Laurencia papillosa (C. Agardh) Greville.

= Palisada papillosa (C. Agardh) K.W. Nam.

Distribución: Veracruz: estuario, litoral, insular (SánchezRodríguez, 1980; Zizumbo Alamilla, 1995; Mateo-Cid et al., 1996, reportada como Laurencia papillosa en las cuatro referencias anteriores; Ortega et al., 2001; De la GarzaFlores, 2003, ambos como Chondrophycus papillosus; Núñez-Resendiz, 2009, como Palisada papillosa; De la Cruz-Francisco et al. 2017); Tabasco: estuario, litoral 
(Ramírez-López, 1996, como Laurencia papillosa; Sentíes y Dreckmann, 2013; Quiroz-González et al., 2018); Campeche: estuario, litoral (Ortega, 1995, como Laurencia papillosa; Ortega et al., 2001; Callejas-Jiménez et al., 2005, ambos como Chondrophycus papillosus; Mateo-Cid et al., 2013b); Yucatán: estuario, litoral, insular (Ortega et al., 2001; Ortegón-Aznar et al., 2001, 2009, las tres referidas como Chondrophycus papillosus; Quintana Roo: estuario, litoral, insular (Mateo-Cid y Mendoza-González, 1991; MendozaGonzález y Mateo-Cid, 1992; Collado-Vides y GonzálezGonzález, 1995; Dreckmann et al., 1996, como Laurencia papillosa; Ortega et al., 2001; Quan-Young et al., 2006; Díaz-Larrea et al., 2007, reportada como Chondrophycus papillosus en las tres citas anteriores; Sentíes y Díaz-Larrea, 2008; Sentíes et al., 2009, como Palisada papillosa; Cassano et al., 2012).

Polysiphonia atlantica Kapraun \& J.N. Norris.

Distribución: Veracruz: estuario, litoral, insular (Ortega et al., 2001; De la Garza-Flores, 2003; Godínez-Ortega et al., 2009); Tabasco: estuario, litoral (Mendoza-González et al., 2017); Campeche: estuario, litoral (Ortega et al., 2001; CaIlejas-Jiménez et al., 2005; Mateo-Cid et al., 2013b; Mendoza-González et al., 2013); Yucatán: estuario, litoral, insular (Ortega et al., 2001; Ortegón-Aznar et al., 2001, 2009); Quintana Roo: estuario, insular (Mateo-Cid y MendozaGonzález, 1991; Collado-Vides y González-González, 1995; Ortega et al., 2001; Mateo-Cid et al., 2006; Mendoza-González et al., 2007).

\section{Polysiphonia binneyi Harvey.}

Distribución: Veracruz: litoral, insular (Ortega et al., 2001; De la Garza-Flores, 2003; Godínez-Ortega et al., 2009); Yucatán: estuario (Ortegón-Aznar et al., 2001); Quintana Roo: estuario, litoral, insular (Mateo-Cid y MendozaGonzález, 1991; Mendoza-González y Mateo-Cid, 1992; Collado-Vides y González-González, 1995; Ortega et al., 2001).

*Polysiphonia breviarticulata var. breviarticulata (C. Agardh) Zanardini.
Distribución: Quintana Roo: litoral, insular (Dreckmann et al., 1996; Ortega et al., 2001).

\section{Polysiphonia breviarticulata var. mexicana Kützing.}

Distribución: Veracruz: litoral (Ortega et al., 2001; De la Garza-Flores, 2003).

*Polysiphonia decussata Hollenberg.

Distribución: Yucatán: insular (Ortega et al., 2001).

*Polysiphonia exilis Harvey.

Distribución: Quintana Roo: estuario, litoral, insular (Mateo-Cid y Mendoza-González, 1991; Collado-Vides y González-González, 1995; Ortega et al., 2001).

\section{Polysiphonia hapalacantha Harvey.}

Distribución: Campeche: litoral (Ortega et al., 2001; MateoCid et al., 2013b).

Polysiphonia havanensis Montagne.

Distribución: Tamaulipas: estuario, litoral (Ortega et al., 2001); Veracruz: litoral, insular (Ortega et al., 2001; De la Garza-Flores, 2003; Godínez-Ortega et al., 2009); Tabasco: litoral (Ramírez-López, 1996; Sentíes y Dreckmann, 2013; Mendoza-González et al., 2017; Quiroz-González et al., 2018); Yucatán: estuario, insular (Ortega et al., 2001; Ortegón-Aznar et al., 2001); Quintana Roo: estuario, litoral, insular (ColladoVides y González-González, 1995; Ortega et al., 2001).

\section{Polysiphonia howei Hollenberg.}

Distribución: Tamaulipas: estuario, litoral (Ortega et al., 2001); Veracruz: litoral (De la Garza-Flores, 2003); Campeche: estuario, litoral (Ortega et al., 2001); Yucatán: litoral (Ortegón-Aznar et al., 2009); Quintana Roo: estuario, litoral, insular (Mateo-Cid y Mendoza-González, 1991; Mendoza-González y Mateo-Cid, 1992; Collado-Vides y GonzálezGonzález, 1995; Ortega et al., 2001). 
*Polysiphonia mollis J.D. Hooker \& Harvey.

Distribución: Yucatán: estuario (Ortegón-Aznar et al., 2001); Quintana Roo: litoral (Dreckmann et al., 1996).

*Polysiphonia opaca (C. Agardh) Moris \& De Notaris.

Distribución: Quintana Roo: litoral (Ortega et al., 2001).

Polysiphonia ramentacea Harvey.

Distribución: Veracruz: estuario, litoral (Ortega et al., 2001; De la Garza-Flores, 2003); Quintana Roo: litoral (Cetz-Navarro et al., 2008).

\section{*Polysiphonia scopulorum Harvey.}

Distribución: Quintana Roo: litoral (Dreckmann et al., 1996; Ortega et al., 2001).

Polysiphonia sertularioides (Grateloup) J. Agardh.

三 Neosiphonia sertularioides (Grateloup) K.W. Nam \& P.J. Kang.

= Polysiphonia flaccidissima Hollenberg.

Distribución: Tabasco: litoral (Ramírez-López, 1996; Sentíes y Dreckmann, 2013, como Neosiphonia sertularioides; Quiroz-González et al., 2018); Campeche: litoral (MateoCid et al., 2013a, como Neosiphonia sertularioides); Quintana Roo: estuario, litoral, insular (Collado-Vides y González-González, 1995, como Polysiphonia flaccidissima; Dreckmann et al., 1996; Ortega et al., 2001; Mateo-Cid et al., 2006, como Polysiphonia flaccidissima).

\section{Polysiphonia subtilissima Montagne.}

Distribución: Tamaulipas: litoral (Ortega et al., 2001); Veracruz: estuario, litoral, insular (Sánchez-Rodríguez, 1980; Ortega et al., 2001; De la Garza-Flores, 2003; Núñez-Resendiz, 2009; De la Cruz-Francisco et al. 2017); Tabasco: estuario, litoral (Ramírez-López, 1996; Sentíes y Dreckmann, 2013; Mendoza-González et al., 2017; Quiroz-González et al., 2018); Campeche: estuario, litoral (Ortega et al., 2001;
Mateo-Cid et al., 2013b); Yucatán: estuario, litoral, insular (Ortega et al., 2001; Ortegón-Aznar et al., 2001, 2009); Quintana Roo: estuario, litoral, insular (Collado-Vides y González-González, 1995; Díaz-Martín y Quan-Young, 2001; Ortega et al., 2001; Quan-Young et al., 2006).

\section{Polysiphonia villum J. Agardh.}

इPolysiphonia scopulorum var. villum (J. Agardh) Hollenberg.

Distribución: Veracruz: insular (Zizumbo Alamilla, 1995); Tabasco: litoral (Mendoza-González et al., 2017, como Polysiphonia scopulorum var. villum); Quintana Roo: litoral, insular (Ortega et al., 2001, como Polysiphonia scopulorum var. villum).

Vertebrata foetidissima (Cocks ex Bornet) Díaz-Tapia \& Maggs.

ミPolysiphonia foetidissima Cocks ex Bornet.

= Polysiphonia tepida Hollenberg.

Distribución: Tamaulipas: litoral (Ortega et al., 2001, como Polysiphonia foetidissima y Polysiphonia tepida); Campeche: estuario, litoral (Ortega et al., 2001; Mateo-Cid et al., 2013a, como Polysiphonia foetidissima).

Wilsonosiphonia howei (Hollenberg) D. Bustamante, Won \& T.O. Cho.

$\equiv$ Neosiphonia howei (Hollenberg) Skelton \& G.R. South. Beih.

Distribución: Campeche: litoral (Mateo-Cid et al., 2013b, como Neosiphonia howei).

Wrightiella blodgettii (Harvey) F. Schmitz.

Distribución: Tamaulipas: litoral (Ortega et al., 2001); Veracruz: litoral (González-Gándara et al., 2007); Quintana Roo: litoral (Ortega et al., 2001).

Wrightiella tumanowiczii (Gatty ex Harvey) F. Schmitz.

Distribución: Campeche: litoral (Mateo-Cid et al., 2013a); Quintana Roo: litoral, insular (Ortega et al., 2001; Vilchis et al., 2018). 
Xiphosiphonia pennata (C. Agardh) Savoie \& G.W. Saunders. इ Pterosiphonia pennata (C. Agardh) Sauvageau.

Distribución: Tamaulipas: litoral (Ortega et al., 2001, como Pterosiphonia pennata); Veracruz: litoral (Núñez-Resendiz, 2009, como Pterosiphonia pennata).

Yuzurua iridescens (M.J. Wynne \& D.L. Ballantine) Sentíes \& M.J. Wynne.

Distribución: Quintana Roo: insular (Sentíes et al., 2015).

Yuzurua poiteaui var. poiteaui (J.V. Lamouroux) MartinLescanne.

इ Laurencia poiteaui (J.V. Lamouroux) M. Howe.

इChondrophycus poiteaui (J.V. Lamouroux) K.W. Nam.

इ Palisada poiteaui (J.V. Lamouroux) K.W. Nam.

Distribución: Tamaulipas: litoral (Ortega et al., 2001, como Laurencia poiteaui); Veracruz: litoral, insular (Zizumbo Alamilla, 1995, como Chondrophycus poiteaui y Laurencia poiteaui; Mateo-Cid et al., 1996; Ortega et al., 2001, ambas como Laurencia poiteaui; De la Garza-Flores, 2003; González-Gándara et al., 2007, ambas como Chondrophycus poiteaui; Godínez-Ortega et al., 2009, como Palisada poiteaui; Galicia-García et al., 2013); Campeche: litoral (Mateo-Cid et al., 2013b); Yucatán: estuario, insular (Ortega et al., 2001, como Laurencia poiteaui; Ortegón-Aznar et al., 2001, 2009); Quintana Roo: estuario, litoral, insular (Mateo-Cid y Mendoza-González, 1991; Mendoza-González y Mateo-Cid, 1992; Dreckmann et al., 1996; Ortega et al., 2001, como Laurencia poiteaui; Sentíes y Fujii, 2002, como Chondrophycus poiteaui; Mateo-Cid et al., 2006; Quan-Young et al., 2006; Díaz-Larrea et al., 2007, como Chondrophycus poiteaui; Mendoza-González et al., 2007; Cassano et al., 2012; Sentíes et al., 2015; Vilchis et al., 2018).

Yuzurua poiteaui var. gemmifera (Harvey) M.J. Wynne.

三 Laurencia gemmifera Harvey.

三 Chondrophycus gemmifer (Harvey) Garbary \& J.T. Harper.

$\equiv$ Chondrophycus poiteaui var. gemmiferus (Harvey) Sen-

tíes, M.T. Fujii \& Díaz.

इ Palisada gemmifera (Harvey) K.W. Nam.
Distribución: Veracruz: litoral, insular (Ortega et al., 2001, como Chondrophycus gemmifera; De la Garza-Flores, 2003); Tabasco: litoral (Mendoza-González et al., 2017); Campeche: estuario, litoral (Ortega, 1995, como Laurencia gemmifera; Ortega et al., 2001; Mateo-Cid et al., 2013b); Quintana Roo: estuario, litoral, insular (Mateo-Cid y Mendoza-González, 1991; Collado-Vides y GonzálezGonzález, 1995, ambas como Laurencia gemmifera; Ortega et al., 2001, como Chondrophycus gemmifera; Sentíes y Fujii, 2002, como Chondrophycus gemmiferus; Díaz-Larrea et al., 2007, como Chondrophycus poiteaui var. gemmiferus; Sentíes et al., 2015; Núñez-Resendiz et al., 2019b).

Wrangeliaceae

Anotrichium tenue (C. Agardh) Nägeli. 三 Griffithsia tenuis C. Agardh.

Distribución: Veracruz: litoral, insular (Ortega et al., 2001; De la Garza-Flores, 2003); Campeche: estuario (Ortega, 1995; Ortega et al., 2001; Mateo-Cid et al., 2013a, b; Mendoza-González et al., 2013); Yucatán: estuario, litoral, insular (Ortega et al., 2001; Ortegón-Aznar et al., 2009); Quintana Roo: estuario, litoral, insular (Mateo-Cid y Mendoza-González, 1991; Mendoza-González y Mateo-Cid, 1992; ColladoVides y González-González, 1995, como Griffithsia tenuis; Dreckmann et al., 1996; Ortega et al., 2001; Mateo-Cid et al., 2006; Mendoza-González et al., 2007).

*Grallatoria reptans M. Howe.

Distribución: Quintana Roo: litoral (Dreckmann et al., 1996; Ortega et al., 2001).

*Griffithsia caribaea G. Feldmann.

Distribución: Quintana Roo: litoral (Ortega et al., 2001).

Griffithsia globulifera Harvey ex Kützing.

Distribución: Veracruz: litoral (Sánchez-Rodríguez, 1980; Ortega et al., 2001; De la Garza-Flores, 2003; Núñez-Re- 
sendiz, 2009); Quintana Roo: litoral, insular (Dreckmann et al., 1996; Díaz-Martín y Quan-Young, 2001; Ortega et al., 2001; Mateo-Cid et al., 2006; Quan-Young et al., 2006).

\section{Griffithsia heteromorpha Kützing.}

Distribución: Campeche: litoral (Mateo-Cid et al., 2013a).

\section{Griffithsia radicans Kützing.}

Distribución: Campeche: litoral (Ortega et al., 2001); Quintana Roo: litoral, insular (Mateo-Cid et al., 2006; Cetz-Navarro et al., 2008).

\section{Griffithsia schousboei Montagne.}

Distribución: Quintana Roo: insular (Ortega et al., 2001; Mendoza-González et al., 2007).

\section{Haloplegma duperreyi Montagne.}

Distribución: Quintana Roo: insular (Mateo-Cid y MendozaGonzález, 1991; Ortega et al., 2001; Mateo-Cid et al., 2006; Mendoza-González et al., 2007).

Pleonosporium boergesenii (A.B. Joly) R.E. Norris.

Distribución: Veracruz: litoral (Mateo-Cid et al., 2018).

Ptilothamnion speluncarum (Collins \& Hervey) D.L. Ballantine \& M.J. Wynne.

इSpermothamnion speluncarum (Collins \& Hervey) M. Howe.

Distribución: Tamaulipas: litoral (Ortega et al., 2001); Veracruz: estuario, litoral (Sánchez-Rodríguez, 1980, como Spermothamnion speluncarum; Ortega et al., 2001; De la Garza-Flores, 2003; Núñez-Resendiz, 2009); Campeche: litoral (Mateo-Cid et al., 2013b); Quintana Roo: insular (Ortega et al., 2001).
*Spermothamnion gymnocarpum M. Howe.

Distribución: Quintana Roo: litoral, insular (Mateo-Cid y Mendoza-González, 1991; Dreckmann et al., 1996; Ortega et al., 2001).

Spermothamnion investiens (P. Crouan \& H. Crouan) Vickers.

Distribución: Yucatán: insular (Ortega et al., 2001); Quintana Roo: litoral, insular (Ortega et al., 2001; Mateo-Cid et al., 2006; Mendoza-González et al., 2007).

Spermothamnion macromeres Collins \& Hervey.

Distribución: Yucatán: insular (Ortega et al., 2001); Quintana Roo: insular (Mateo-Cid y Mendoza-González, 1991; Ortega et al., 2001).

Spongoclonium caribaeum (Børgesen) M.J. Wynne. 三Pleonosporium caribaeum (Børgesen) R.E. Norris.

Distribución: Tamaulipas: litoral (Ortega et al., 2001, como Pleonosporium caribaeum); Veracruz: litoral (Mateo Cid et al., 2018); Campeche: litoral (Mendoza-González et al., 2013).

Tiffaniella gorgonea (Montagne) Doty \& Meñez.

Distribución: Tabasco: litoral (Sentíes y Dreckmann, 2013; Quiroz-González et al., 2018); Campeche: litoral (MateoCid et al., 2013a); Quintana Roo: insular (Mateo-Cid y Mendoza-González, 1991; Mendoza-González y Mateo-Cid, 1992; Ortega et al., 2001; Mendoza-González et al., 2007).

Wrangelia argus (Montagne) Montagne.

Distribución: Veracruz: estuario, litoral, insular (SánchezRodríguez, 1980; Ortega et al., 2001; De la Garza-Flores, 2003; González-Gándara et al., 2007; Núñez-Resendiz, 2009; De la Cruz-Francisco et al. 2017); Tabasco: litoral (Mendoza-González et al., 2017); Campeche: estuario, 
litoral (Ortega, 1995; Ortega et al., 2001; Callejas-Jiménez et al., 2005; Mateo-Cid et al., 2013b); Yucatán: insular (Ortega et al., 2001); Quintana Roo: litoral, insular (Mateo-Cid y Mendoza-González, 1991; Mendoza-González y Mateo-Cid, 1992; Dreckmann et al., 1996; Ortega et al., 2001; Quan-Young et al., 2006; Mateo-Cid et al., 2006).

\section{Wrangelia bicuspidata Børgesen.}

Distribución: Yucatán: insular (Ortega et al., 2001); Quintana Roo: litoral, insular (Mateo-Cid y Mendoza-González, 1991; Dreckmann et al., 1996; Ortega et al., 2001; QuanYoung et al., 2006; Mateo-Cid et al., 2006; Mendoza-González et al., 2007).

Wrangelia penicillata (C. Agardh) C. Agardh.

Distribución: Veracruz: litoral (Ortega et al., 2001; De la Garza-Flores, 2003); Campeche: litoral (Mateo-Cid et al., 2013a); Quintana Roo: litoral, insular (Mateo-Cid y Mendoza-González, 1991; Mendoza-González y Mateo-Cid, 1992; Dreckmann et al., 1996; Ortega et al., 2001; Mateo-Cid et al., 2006; Quan-Young et al., 2006).

\section{Colaconematales}

\section{Colaconemataceae}

*Colaconema bisporum (Børgesen) I.K. Hwang \& H.S. Kim. इAcrochaetium bisporum (Børgesen) Børgesen.

Distribución: Quintana Roo: litoral (Ortega et al., 2001, como Acrochaetium bisporum).

Colaconema dasyae (Collins) Stegenga.

Distribución: Tabasco: litoral (Mendoza-González et al., 2017).

Colaconema daviesii (Dillwyn) Stegenga. इ Acrochaetium daviesii (Dillwyn) Nägeli. इ Audouinella daviesii (Dillwyn) Woelkerling.
Distribución: Tabasco: litoral (Mendoza-González et al., 2017); Campeche: litoral (Mateo-Cid et al., 2013b); Quintana Roo: litoral, insular (Mateo-Cid y Mendoza-González, 1991, como Acrochaetium daviesii; Mendoza-González y Mateo-Cid, 1992, ambos como Audouinella daviesii; Ortega et al., 2001, como Acrochaetium daviesii).

Colaconema hallandicum (Kylin) Afonso-Carillo, Sanson, Sangil \& Díaz-Villa.

= Acrochaetium dufourii Børgesen.

इ Acrochaetium hallandicum (Kylin) Hamel.

इ Audouinella hallandica (Kylin) Woelkerling.

Distribución: Tamaulipas: litoral (Ortega et al., 2001, como Acrochaetium hallandicum); Veracruz: estuario, litoral (Sánchez-Rodríguez, 1980, como Acrochaetium dufourii; Ortega et al., 2001; De la Garza-Flores, 2003, ambas como Acrochaetium hallandicum; Núñez-Resendiz, 2009; GaliciaGarcía et al., 2013); Tabasco: litoral (Mendoza-González et al., 2017); Campeche: litoral (Ortega et al., 2001, como Acrochaetium hallandicum; Mateo-Cid et al., 2013b; Mendoza-González et al., 2013); Quintana Roo: insular (Mateo-Cid y Mendoza-González, 1991, como Audouinella hallandica; Ortega et al., 2001; Mateo-Cid et al., 2006, ambas como Acrochaetium hallandicum).

Colaconema hypneae (Børgesen) A.A. Santos \& C.W.N. Moura.

इ Acrochaetium hypneae (Børgesen) Børgesen.

इ Audouinella hypneae (Børgesen) G. Lawson \& D.M. John. Beih.

Distribución: Tamaulipas: estuario, litoral (Ortega et al., 2001, como Acrochaetium hypneae); Veracruz: litoral, insular (Ortega et al., 2001; De la Garza-Flores, 2003, ambas como Acrochaetium hypneae); Tabasco: estuario, litoral (Mendoza-González et al., 2017); Campeche: estuario, litoral (Ortega, 1995, como Audouinella hypneae; Ortega et al., 2001; Mateo-Cid et al., 2013b, reportada como Acrochaetium hypneae en las tres citas previas; MendozaGonzález et al., 2013); Quintana Roo: litoral, insular (Mateo-Cid et al., 2006; Cetz-Navarro et al., 2008, ambas como Acrochaetium hypneae). 
*Colaconema robustum (Børgesen) Huisman \& Woelkerling. 三 Acrochaetium robustum Børgesen.

Distribución: Yucatán: insular (Ortega et al., 2001, como Acrochaetium robustum).

Colaconema savianum (Meneghini) R. Nielsen.

ミAcrochaetium savianum (Meneghini) Nägeli.

Distribución: Veracruz: estuario, litoral (Ortega et al., 2001; De la Garza-Flores, 2003, ambas como Acrochaetium savianum); Tabasco: estuario, litoral (Mendoza-González et al., 2017).

Corallinales

Corallinoideae

Corallina officinalis Linnaeus.

Distribución: Veracruz: litoral (De la Cruz-Francisco et al. 2017).

Jania adhaerens J.V. Lamouroux.

Distribución: Tamaulipas: estuario, litoral (Ortega et al., 2001); Veracruz: estuario, litoral, insular (Sánchez-Rodríguez, 1980; Zizumbo Alamilla, 1995; Mateo-Cid et al., 1996; Ortega et al., 2001; De la Garza-Flores, 2003; GonzálezGándara et al., 2007; Núñez-Resendiz, 2009; Galicia-García et al., 2013; Landa-Cansigno et al., 2019); Tabasco: litoral (Mendoza-González et al., 2017; Quiroz-González et al., 2018); Campeche: estuario, litoral (Ortega, 1995; Ortega et al., 2001; Mateo-Cid et al., 2013b; Mendoza-González et al., 2013); Yucatán: estuario, litoral, insular (Ortega et al., 2001; Ortegón-Aznar et al., 2009); Quintana Roo: estuario, litoral, insular (Mateo-Cid y Mendoza-González, 1991; Mendoza-González y Mateo-Cid, 1992; Collado-Vides y González-González, 1995; Dreckmann et al., 1996; Ortega et al., 2001; Quan-Young et al., 2006; Mateo-Cid et al., 2006; Mendoza-González et al., 2007).
Jania capillacea Harvey.

Distribución: Tamaulipas: litoral (Ortega et al., 2001; Vilchis et al., 2018); Veracruz: estuario, litoral, insular (Ortega et al., 2001; De la Garza-Flores, 2003; Godínez-Ortega et al., 2009; Vilchis et al., 2018); Tabasco: litoral: (Mendoza-González et al., 2017); Campeche: estuario, litoral (Ortega et al., 2001; Callejas-Jiménez et al., 2005); Yucatán: litoral, insular (Ortega et al., 2001; Vilchis et al., 2018); Quintana Roo: estuario, litoral, insular (Ortega et al., 2001; Quan-Young et al., 2006; Mateo-Cid et al., 2006; Mendoza-González et al., 2007; Vilchis et al., 2018).

Jania cubensis Montagne ex Kützing.

三 Corallina cubensis (Montagne ex Kützing) Kützing.

ミ Haliptilon cubense (Montagne ex Kützing) Garbary \& H.W. Johansen.

Distribución: Tamaulipas: litoral (Ortega et al., 2001; Vargas López et al., 2004, ambas como Haliptilon cubense); Veracruz: litoral, insular (Sánchez-Rodríguez, 1980, como Corallina cubensis; Ortega et al., 2001; De la Garza-Flores, 2003, ambas previas como Haliptilon cubense; Galicia-García et al., 2013; Landa-Cansigno et al., 2019); Tabasco: litoral (Sentíes y Dreckmann, 2013; Quiroz-González et al., 2018); Campeche: litoral (Ortega et al., 2001, como Haliptilon cubense; Mateo-Cid et al., 2013a, b; Mendoza-González et al., 2013); Yucatán: litoral, insular (Ortega et al., 2001; Ortegón-Aznar et al., 2009) ambas como Haliptilon cubense; Quintana Roo: litoral, insular (Mateo-Cid y Mendoza-González, 1991; Mendoza-González y Mateo-Cid, 1992; Dreckmann et al., 1996; Ortega et al., 2001; Quan-Young et al., 2006; Mateo-Cid et al., 2006) como Haliptilon cubense para la región.

Jania pumila J.V. Lamouroux.

Distribución: Campeche: litoral (Callejas-Jiménez et al., 2005; Mateo-Cid et al., 2013a); Yucatán: estuario, litoral, insular (Ortega et al., 2001; Ortegón-Aznar et al., 2001; 
Núñez-Resendiz et al., 2019b); Quintana Roo: litoral, insular (Dreckmann et al., 1996; Ortega et al., 2001; QuanYoung et al., 2006).

Jania rubens (Linnaeus) J.V. Lamouroux.

Distribución: Tamaulipas: litoral (Ortega et al., 2001); Veracruz: estuario, litoral, insular (Sánchez-Rodríguez, 1980; Ortega et al., 2001; De la Garza-Flores, 2003; Godínez-Ortega et al., 2009; Núñez-Resendiz, 2009; Landa-Cansigno et al., 2019); Tabasco: litoral (Mendoza-González et al., 2017); Campeche: litoral (Mateo-Cid et al., 2013b; Mendoza-González et al., 2013); Yucatán: insular (Ortega et al., 2001); Quintana Roo: insular (Mateo-Cid y Mendoza-González, 1991; Ortega et al., 2001; Quan-Young et al., 2006; Mendoza-González et al., 2007).

Jania subulata (Ellis \& Solander) Sonder.

三 Corallina subulata J. Ellis \& Solander.

ミ Haliptilon subulatum (J. Ellis \& Solander) H.W. Johansen.

Distribución: Tamaulipas: litoral (Ortega et al., 2001, como Haliptilon subulatum); Veracruz: estuario, litoral, insular (Sánchez-Rodríguez, 1980, como Corallina subulata; Ortega et al., 2001; De la Garza-Flores, 2003; Núñez-Resendiz, 2009, reportada como Haliptilon subulatum en las tres anteriores citas; Landa-Cansigno et al., 2019); Yucatán: litoral (Ortega et al., 2001, como Haliptilon subulatum); Quintana Roo: litoral, insular (Dreckmann et al., 1996; Díaz-Martín y Quan-Young, 2001; Ortega et al., 2001; Mendoza-González et al., 2007, reportada como Haliptilon subulatum para la región).

Jania tenella (Kützing) Grunow.

Distribución: Veracruz: litoral (De la Garza-Flores, 2003).

Jania ungulata f. brevior (Yendo) Yendo.

Distribución: Tabasco: litoral (Mendoza-González et al., 2017).
Hydrolithaceae

Hydrolithon boergesenii (Foslie) Foslie.

Distribución: Veracruz: litoral, insular (Ortega et al., 2001; De la Garza-Flores, 2003; Godínez-Ortega et al., 2009); Quintana Roo: insular (Mateo-Cid y Mendoza-González, 1991; Ortega et al., 2001; Mateo-Cid et al., 2006).

Hydrolithon boreale (Foslie) Y.M. Chamberlain.

= Fosliella farinosa f. callithamnioides (Falkenberg) Y.M. Chamberlain.

Distribución: Campeche: estuario, litoral (Ortega, 1995, como Fosliella farinosa f. callithamnioides; Mateo-Cid et al., 2013b).

Hydrolithon farinosum (J.V. Lamouroux) Penrose \& Y.M. Chamberlain.

इ Fosliella farinosa (J.V. Lamouroux) M. Howe.

Distribución: Veracruz: litoral, insular (Zizumbo Alamilla, 1995, como Fosliella farinosa; Mateo-Cid et al., 1996; Ortega et al., 2001; De la Garza-Flores, 2003); Tabasco: litoral (Mendoza-González et al., 2017); Campeche: estuario, litoral (Ortega, 1995; Ortega et al., 2001; Callejas-Jiménez et al., 2005, como Fosliella farinosa; Mateo-Cid et al., 2013b); Yucatán: insular (Ortega et al., 2001); Quintana Roo: estuario, litoral, insular (Mateo-Cid y Mendoza-González, 1991; Mendoza-González y Mateo-Cid, 1992, ambas como Fosliella farinosa; Ortega et al., 2001; Mateo-Cid et al., 2006; Mendoza-González et al., 2007).

Pneophyllum confervicola (Kützing) Y.M. Chamberlain.

Distribución: Quintana Roo: litoral, insular (Mateo-Cid y Mendoza-González, 2009).

\section{Pneophyllum fragile Kützing.}

= Fosliella lejolisii (Rosanoff) M. Howe.

= Pneophyllum lejolisii (Rosanoff) Y.M. Chamberlain. 
Distribución: Tamaulipas: litoral (Ortega et al., 2001; Mateo-Cid y Mendoza-González, 2009); Veracruz: estuario, litoral, insular (Sánchez-Rodríguez, 1980; Zizumbo Alamilla, 1995, ambas como Fosliella lejolisii; Mateo-Cid et al., 1996; Ortega et al., 2001; De la Garza-Flores, 2003; Mateo-Cid y Mendoza-González, 2009; Núñez-Resendiz, 2009; GaliciaGarcía et al., 2013); Tabasco: litoral (Sentíes y Dreckmann, 2013; Quiroz-González et al., 2018); Campeche: estuario (Ortega, 1995, como Fosliella lejolisii; Ortega et al., 2001; Mateo-Cid y Mendoza-González, 2009; Mateo-Cid et al., 2013b); Yucatán: litoral, insular (Ortega et al., 2001; MateoCid y Mendoza-González, 2009); Quintana Roo: litoral, insular (Mateo-Cid y Mendoza-González, 1991, como PneophyIlum lejolisii; Mendoza-González y Mateo-Cid, 1992, como Pneophyllum lejolisii; Dreckmann et al., 1996; Ortega et al., 2001; Mateo-Cid et al., 2006).

\section{Lithophylloideae}

\section{Amphiroa beauvoisii J.V. Lamouroux.}

Distribución: Veracruz: litoral (Luna-Ortega y De la CruzFrancisco, 2017); Yucatán: insular (Ortega et al., 2001); Quintana Roo: litoral, insular (Dreckmann et al., 1996; Ortega et al., 2001; Mendoza-González et al., 2007).

\section{Amphiroa brasiliana Decaisne.}

Distribución: Tamaulipas: litoral (Ortega et al., 2001); Veracruz: insular (Luna-Ortega y De la Cruz-Francisco, 2017); Campeche: litoral (Ortega et al., 2001); Quintana Roo: insular (Ortega et al., 2001).

Amphiroa fragilissima (Linnaeus) J.V. Lamouroux.

Distribución: Veracruz: estuario, litoral, insular (SánchezRodríguez, 1980; Zizumbo Alamilla, 1995; Mateo-Cid et al., 1996; Ortega et al., 2001; De la Garza-Flores, 2003; González-Gándara et al., 2007; Godínez-Ortega et al., 2009; Núñez-Resendiz, 2009; Galicia-García et al., 2013); Campeche: litoral (Ortega et al., 2001; Mateo-Cid et al., 2013b; Mendoza-González et al., 2013); Yucatán: estuario (Ortega et al., 2001; Ortegón-Aznar et al., 2001); Quintana Roo: litoral, insular (Mateo-Cid y Mendoza-González, 1991; Mendoza-González y Mateo-Cid, 1992; Dreckmann et al., 1996; Ortega et al., 2001; Quan-Young et al., 2006; Mateo-Cid et al., 2006; Mendoza-González et al., 2007).

Amphiroa hancockii W.R. Taylor.

Distribución: Veracruz: litoral, insular (Ortega et al., 2001; De la Garza-Flores, 2003).

Amphiroa rigida J.V. Lamouroux.

= Amphiroa rigida var. antillana Børgesen.

Distribución: Veracruz: litoral, insular (Mateo-Cid et al., 1996, como Amphiroa rigida var. antillana; Ortega et al., 2001; De la Garza-Flores, 2003; González-Gándara et al., 2007; Godínez-Ortega et al., 2009; Luna-Ortega y De la Cruz-Francisco, 2017); Campeche: litoral (Mateo-Cid et al., 2013a, b); Yucatán: estuario, litoral, insular (Ortega et al., 2001; Ortegón-Aznar et al., 2009); Quintana Roo: estuario, litoral, insular (Mateo-Cid y Mendoza-González, 1991; Mendoza-González y Mateo-Cid, 1992; Dreckmann et al., 1996, como Amphiroa rigida var. antillana en las tres citas anteriores; Ortega et al., 2001; Mateo-Cid et al., 2006; Quan-Young et al., 2006, como Amphiroa rigida var. antillana).

Amphiroa vanbosseae Me. Lemoine.

Distribucion: Campeche: litoral (Mateo-Cid et al., 2013b).

*Goniolithon decutescens (Heydrich) Foslie ex M. Howe.

Distribución: Veracruz: insular (Zizumbo Alamilla, 1995); Quintana Roo: insular (Mateo-Cid y Mendoza-González, 1991; Mendoza-González y Mateo-Cid, 1992).

Lithophyllum corallinae (P. Crouan \& H. Crouan) Heydrich.

Distribución: Veracruz: litoral, insular (García-López et al., 2013). 
*Lithophyllum daedaleum var. pseudodentatum Foslie \& M. Howe.

Distribución: Veracruz: insular (Mateo-Cid et al., 1996).

Lithophyllum incrustans Philippi.

Distribucion: Quintana Roo: insular (García-López et al., 2013).

Lithophyllum intermedium Foslie.

Distribución: Veracruz: litoral, insular (Zizumbo Alamilla, 1995; Mateo-Cid et al., 1996; Ortega et al., 2001; De la Garza-Flores, 2003).

Lithophyllum kaiseri (Heydrich) Heydrich.

= Lithophyllum congestum (Foslie) Foslie.

= Lithophyllum daedaleum Foslie \& M. Howe.

Distribución: Veracruz: litoral, insular (Mateo-Cid et al., 1996, como Lithophyllum daedaleum; Ortega et al., 2001; De la Garza-Flores, 2003, reportada como Lithophyllum congestum para esta región); Campeche: litoral (GarcíaLópez et al., 2013); Yucatán: insular (Ortega et al., 2001).

Lithophyllum prototypum (Foslie) Foslie.

ミTitanoderma prototypum (Foslie) Woelkerling, Y.M.

Chamberlain \& P.C. Silva.

Distribución: Veracruz: insular (García-López et al., 2013); Tabasco: litoral (Mendoza-González et al., 2017); Quintana Roo: litoral, insular (Mateo-Cid y Mendoza-González, 1991, como Titanoderma prototypum; Ortega et al., 2001; GarcíaLópez et al., 2013).

Lithophyllum stictiforme (Areschoug) Hauck.

= Lithophyllum bermudense Foslie \& M. Howe.

= Lithophyllum frondosum (Dufour) G. Furnari, Cormaci \& Alongi

Distribución: Veracruz: litoral, insular (Mateo-Cid et al., 1996, como Lithophyllum bermudense; Ortega et al.,
2001; De la Garza-Flores, 2003, ambas como Lithophyllum frondosum; García-López et al., 2013); Tabasco: litoral (Mendoza-González et al., 2017); Yucatán: insular (GarcíaLópez et al., 2013).

Lithothamnion occidentale (Foslie) Foslie.

Distribución: Veracruz: litoral, insular (Ortega et al., 2001; De la Garza-Flores, 2003); Campeche: litoral (Mateo-Cid et al., 2013a); Yucatán: insular (Ortega et al., 2001); Quintana Roo: litoral, insular (Dreckmann et al., 1996; Ortega et al., 2001; Mendoza-González et al., 2007).

Titanoderma pustulatum (J.V. Lamouroux) Nägeli. ミ Lithophyllum pustulatum (J.V. Lamouroux) Foslie.

Distribución: Tamaulipas: litoral (Ortega et al., 2001); Veracruz: litoral, insular (Ortega et al., 2001; De la Garza-Flores, 2003); Campeche: litoral (Mateo-Cid et al., 2013b, como Lithophyllum pustulatum); Quintana Roo: litoral, insular (Mateo-Cid et al., 2006; Cetz-Navarro et al., 2008).

\section{Mastoporaceae}

Lithoporella atlantica (Foslie) Foslie.

Distribución: Yucatán: insular (Ortega et al., 2001); Quintana Roo: insular (Mendoza-González et al., 2007).

Lithoporella bermudensis (Foslie) W.H. Adey.

Distribución: Quintana Roo: insular (Ortega et al., 2001; Mateo-Cid et al., 2006; Mendoza-González et al., 2007).

Porolithaceae

Porolithon antillarum (Foslie \& M. Howe) Foslie \& M. Howe. = Porolithon pachydermum (Foslie) Foslie.

Distribución: Veracruz: litoral, insular (Ortega et al., 2001; De la Garza-Flores, 2003; Galicia-García et al., 2013); Quintana Roo: insular (Mateo-Cid y Mendoza-González, 1991; Mendoza-González y Mateo-Cid, 1992; Ortega et al., 
2001). Reportada para ambas regiones como Porolithon pachydermum.

Porolithon improcerum (Foslie \& M. Howe) M. Howe. 三 Hydrolithon improcerum (Foslie \& M. Howe) Foslie.

Distribución: Veracruz: litoral, insular (Zizumbo AlamiIla, 1995; Mateo-Cid et al., 1996; Ortega et al., 2001; De la Garza-Flores, 2003); Quintana Roo: insular (Mateo-Cid y Mendoza-González, 1991; Mendoza-González y MateoCid, 1992; Ortega et al., 2001). En ambas regiones como Hydrolithon improcerum.

Spongitaceae

Neogoniolithon accretum (Foslie \& M. Howe) Setchell \& L.R. Mason.

Distribución: Veracruz: litoral, insular (Zizumbo Alamilla, 1995; Ortega et al., 2001; De la Garza-Flores, 2003; Mateo-Cid et al., 2014b); Campeche: litoral (Mateo-Cid et al., 2013a); Yucatán: insular (Mateo-Cid et al., 2014b); Quintana Roo: litoral, insular (Mateo-Cid y Mendoza-González, 1991; Mendoza-González y Mateo-Cid, 1992; Ortega et al., 2001; Mateo-Cid et al., 2006, 2014b).

Neogoniolithon acropetum (Foslie \& M. Howe) W.H. Adey.

Distribución: Yucatán: insular (Mateo-Cid et al., 2014b); Quintana Roo: litoral, insular (Mendoza-González y MateoCid, 1992; Ortega et al., 2001; Mateo-Cid et al., 2014b).

Neogoniolithon affine (Foslie \& M. Howe) Setchell \& L.R. Mason.

Distribución: Yucatán: insular (Ortega et al., 2001); Quintana Roo: insular (Mateo-Cid y Mendoza-González, 1991; Mendoza-González y Mateo-Cid, 1992; Ortega et al., 2001).

Neogoniolithon brassica-florida (Harvey) Setchell \& L. R. Mason.

Distribucion: Campeche: litoral (Mateo-Cid et al., 2013a).
Neogoniolithon erosum (Foslie) W.H. Adey.

Distribución: Veracruz: litoral, insular (Ortega et al., 2001; De la Garza-Flores, 2003; Mateo-Cid et al., 2014b); Quintana Roo: litoral, insular (Mateo-Cid y Mendoza-González, 1991; Mendoza-González y Mateo-Cid, 1992; Ortega et al., 2001; Mateo-Cid et al., 2014b).

Neogoniolithon fosliei (Heydrich) Setchell \& L.R. Mason.

= Neogoniolithon solubile (Foslie \& M. Howe) Setchell \& L.R. Mason.

= Paragoniolithon solubile (Foslie \& M. Howe) W.H. Adey, R.A. Townsend \& Boykins.

Distribución: Veracruz: litoral, insular (Mateo-Cid et al., 1996, como Paragoniolithon solubile, Mateo-cid., 2014b; como Neogoniolithon solubile, Ortega et al., 2001; como Neogoniolithon solubile, De la Garza-Flores, 2003); Yucatán: insular (Mateo-Cid et al., 2014b, como Neogoniolithon solubile); Quintana Roo: litoral, insular (Ortega et al., 2001; Mateo-Cid et al., 2006, como Paragoniolithon solubile, Mateo-Cid et al., 2014b; como Neogoniolithon solubile, Mendoza-González et al., 2007; Cetz-Navarro et al., 2008).

Neogoniolithon mamillare (Harvey) Setchell \& L.R. Mason.

Distribución: Veracruz: litoral, insular (Mateo-Cid et al., 1996; Ortega et al., 2001; De la Garza-Flores, 2003); Quintana Roo: estuario, litoral, insular (Mateo-Cid et al., 2006, 2014b; Cetz-Navarro et al., 2008).

Neogoniolithon propinquum (Foslie) Me. Lemoine.

Distribución: Veracruz: insular (Mateo-Cid et al., 2014b); Yucatán: insular (Mateo-Cid et al., 2014b); Quintana Roo: litoral, insular (Mateo-Cid et al., 2014b).

Neogoniolithon rhizophorae (Foslie \& M. Howe) Setchell \& L.R. Mason.

Distribución: Veracruz: litoral, insular (Mateo-Cid et al., 2014b); Yucatán: insular (Mateo-Cid et al., 2014b); Quinta- 
na Roo: litoral, insular (Mateo-Cid et al., 2014b).

Neogoniolithon siankanensis illeg. L.E. Mateo-Cid, A.C. Mendoza-González \& P.W. Gabrielson.

Distribución: Yucatán: insular (Mateo-Cid et al., 2014b); Quintana Roo: litoral (Mateo-Cid et al., 2014b).

Neogoniolithon spectabile (Foslie) Setchell \& L.R. Mason.

Distribución: Veracruz: litoral, insular (Mateo-Cid et al., 1996; Ortega et al., 2001; De la Garza-Flores, 2003); Yucatán: insular (Ortega et al., 2001; Mateo-Cid et al., 2014b); Quintana Roo: litoral, insular (Mateo-Cid y Mendoza-González, 1991; Mendoza-González y Mateo-Cid, 1992; Ortega et al., 2001; Mateo-Cid et al., 2014b).

Neogoniolithon strictum (Foslie) Setchell \& L.R. Mason.

Distribución: Veracruz: litoral, insular (Ortega et al., 2001; De la Garza-Flores, 2003); Yucatán: insular (Ortega et al., 2001; Mateo-Cid et al., 2014b); Quintana Roo: litoral, insular (Mateo-Cid y Mendoza-González, 1991; Dreckmann et al., 1996; Ortega et al., 2001; Mateo-Cid et al., 2006, 2014b).

Neogoniolithon trichotomum (Heydrich) Setchell \& L.R. Mason.

Distribución: Yucatán: insular (Mendoza-González y MateoCid, 2007); Quintana Roo: litoral, insular (Mendoza-González y Mateo-Cid, 2007).

Spongites absimilis (Foslie \& M. Howe) Afonso-Carrillo.

Distribución: Veracruz: litoral, insular (Ortega et al., 2001; De la Garza-Flores, 2003).

Spongites yendoi (Foslie) Y.M. Chamberlain.

Distribución: Campeche: litoral (Mateo-Cid et al., 2013a,b).
Gelidiales

Gelidiellaceae

Gelidiella acerosa (Forsskål) Feldmann \& Hamel.

Distribución: Veracruz: litoral, insular (Zizumbo Alamilla, 1995; Mateo-Cid et al., 1996; Ortega et al., 2001; De la Garza-Flores, 2003; Núñez-Resendiz, 2009; De la Cruz-Francisco et al., 2017; Landa-Cansigno et al., 2019); Campeche: litoral (Ortega et al., 2001; Mateo-Cid et al., 2013a, b); Yucatán: insular (Ortega et al., 2001); Quintana Roo: litoral, insular (Mateo-Cid y Mendoza-González, 1991; Mendoza-González y Mateo-Cid, 1992; Dreckmann et al., 1996; Ortega et al., 2001; Mateo-Cid et al., 2006).

Gelidiella lubrica (Kützing) Feldmann \& Hamel.

Distribución: Yucatán: insular (Ortega et al., 2001); Quintana Roo: litoral (Cetz-Navarro et al., 2008).

Gelidium americanum (W.R. Taylor) Santelices.

E Pterocladia americana W.R. Taylor.

Distribución: Tamaulipas: litoral (Ortega et al., 2001; Vargas López et al., 2004); Veracruz: litoral, insular (SánchezRodríguez, 1980; como Pterocladia americana, Ortega et al., 2001; De la Garza-Flores, 2003; Núñez-Resendiz, 2009); Tabasco: estuario, litoral (Mendoza-González et al., 2017); Campeche: estuario, litoral (Ortega, 1995; Ortega et al., 2001; Mateo-Cid et al., 2013b); Quintana Roo: litoral, insular (Mateo-Cid y Mendoza-González, 1991; Mendoza-González y Mateo-Cid, 1992; Dreckmann et al., 1996; Ortega et al., 2001; Mendoza-González et al., 2007).

Gelidium corneum (Hudson) J.V. Lamouroux.

Distribución: Veracruz: litoral, insular (Ortega et al., 2001; De la Garza-Flores, 2003; Landa-Cansigno et al., 2019); Tabasco: estuario, litoral (Mendoza-González et al., 2017); Campeche: litoral (Mendoza-González et al., 2013); Quintana Roo: insular (Ortega et al., 2001). 
Gelidium crinale (Hareex Turner) Gaillon.

Distribución: Tamaulipas: litoral (Ortega et al., 2001); Veracruz: litoral, insular (Ortega et al., 2001; De la Garza-Flores, 2003); Campeche: estuario, litoral (Ortega et al., 2001; Mateo-Cid et al., 2013b; Mendoza-González et al., 2013); Yucatán: insular (Ortega et al., 2001); Quintana Roo: insular (Mateo-Cid y Mendoza-González, 1991; Ortega et al., 2001).

\section{Gelidium floridanum W.R. Taylor.}

Distribución: Veracruz: (litoral) (Sánchez-Rodríguez, 1980; Ortega et al., 2001; De la Garza-Flores, 2003).

Gelidium pusillum (Stackhouse) Le Jolis.

Distribución: Tamaulipas: litoral (Vargas López et al., 2004; Vilchis et al., 2018); Veracruz: litoral, insular (Ortega et al., 2001; De la Garza-Flores, 2003; Godínez-Ortega et al., 2009; De la Cruz-Francisco et al. 2017; Vilchis et al., 2018); Tabasco: estuario, litoral (Ramírez-López, 1996; Sentíes y Dreckmann, 2013; Mendoza-González et al., 2017; QuirozGonzález et al., 2018; Vilchis et al., 2018); Campeche: estuario, litoral (Ortega et al., 2001; Mateo-Cid et al., 2013b; Vilchis et al., 2018); Yucatán: litoral, insular (Ortega et al., 2001; Vilchis et al., 2018); Quintana Roo: litoral, insular (Mateo-Cid y Mendoza-González, 1991; Mendoza-González y Mateo-Cid, 1992; Ortega, 1995; Dreckmann et al., 1996; Ortega et al., 2001; Mateo-Cid et al., 2006).

*Gelidium spinosum (S.G. Gmelin) P.C. Silva. = Gelidium latifolium Bornet ex Hauck.

Distribución: Tamaulipas: estuario, litoral (Ortega et al., 2001); Quintana Roo: insular (Mateo-Cid y Mendoza-González, 1991; Mendoza-González y Mateo-Cid, 1992, ambas como Gelidium latifolium; Ortega et al., 2001).

Millerella pannosa (Feldmann) G.H. Boo \& L. LeGall. 三 Gelidiella pannosa (Feldmann) Feldmann \& Hamel. = Gelidiella tenuissima Feldmann \& Hamel.

Distribución: Veracruz: litoral (Sánchez-Rodríguez, 1980, como Gelidiella tenuissima; Ortega et al., 2001; De la GarzaFlores, 2003, ambas como Gelidiella pannosa); Quintana Roo: insular (Ortega et al., 2001, como Gelidiella pannosa).

Parviphycus setaceus (Feldmann) J. Afonso-Carrillo, M. Sanson, C. Sangil \& T. Díaz-Villa. 三 Gelidiella setacea (Feldmann) Feldmann \& Hamel.

Distribución: Veracruz: litoral (De la Garza-Flores, 2003, como Gelidiella setacea); Campeche: litoral (Mateo-Cid et al., 2013b).

Parviphycus trinitatensis (W.R. Taylor) M.J. Wynne. 三 Gelidiella trinitatensis W.R. Taylor.

Distribución: Veracruz: estuario, litoral, insular (SánchezRodríguez, 1980; Ortega et al., 2001; De la Garza-Flores, 2003, reportada como Gelidiella trinitatensis en las tres anteriores citas; Luna-Ortega y De la Cruz-Francisco, 2017); Quintana Roo: litoral, insular (Mendoza-González y MateoCid, 1992; Ortega et al., 2001, ambas como Gelidiella trinitatensis).

Pterocladiaceae

Pterocladiella caerulescens (Kützing) Santelices \& Hommersand.

इPterocladia caerulescens (Kützing) Santelices.

Distribución: Veracruz: litoral, insular (Mateo-Cid et al., 1996, como Pterocladia caerulescens; Ortega et al., 2001; De la Garza-Flores, 2003); Yucatán: insular (Ortega et al., 2001); Quintana Roo: insular (Ortega et al., 2001).

Pterocladiella bartlettii (W.R. Taylor) Santelices.

= Gelidiella taylorii A.B. Joly.

इ Pterocladia bartlettii W.R. Taylor.

= Pterocladiella taylorii (Joly) Santelices.

Distribución: Veracruz: litoral, insular (Ortega et al., 2001, como Gelidiella taylorii; De la Garza-Flores, 2003, ambas como Pterocladia bartlettii); Campeche: litoral (Ortega et al., 2001, como Gelidiella taylorii y Pterocladia bartlettii; 
Mateo-Cid et al., 2013b, como Pterocladiella taylorii); Yucatán: insular (Ortega et al., 2001, como Gelidiella taylorii y Pterocladia bartlettii).

*Pterocladiella caloglossoides (M. Howe) Santelices. 三Pterocladia caloglossoides (M. Howe) E.Y. Dawson.

Distribución: Quintana Roo: insular (Ortega et al., 2001, como Pterocladia caloglossoides).

Pterocladiella capillacea (S.G. Gmelin) Santelices \& Hommersand.

इ Pterocladia capillacea (S.G. Gmelin) Bornet.

= Pterocladia pinnata (Hudson) Papenfuss.

Distribución: Tamaulipas: litoral (Ortega et al., 2001; Vargas López et al., 2004, como Pterocladia capillacea); Veracruz: litoral (Sánchez-Rodríguez, 1980, como Pterocladia pinnata; Ortega et al., 2001; De la Garza-Flores, 2003); Campeche: litoral (Ortega et al., 2001; Mateo-Cid et al., 2013a, b); Quintana Roo: litoral, insular (Mateo-Cid y Mendoza-González, 1991; Dreckmann et al., 1996, ambas como Pterocladia capillacea; Ortega et al., 2001).

Pterocladiella sanctarum (Feldmann \& Hamel) Santelices. 三 Gelidiella sanctarum Feldmann \& Hamel.

Distribución: Veracruz: insular (Luna-Ortega y De la CruzFrancisco, 2017); Tabasco: litoral (Mendoza-González et al., 2017); Campeche: litoral (Mateo-Cid et al., 2013a, b); Yucatán: insular (Ortega et al., 2001, como Gelidiella sanctarum); Quintana Roo: insular (Ortega et al., 2001, como Gelidiella sanctarum).

Gigartinales

\section{Caulacanthaceae}

Catenella caespitosa (Withering) L.M. Irvine.

Distribución: Campeche: litoral (Ortega et al., 2001; MateoCid et al., 2013b); Quintana Roo: litoral, insular (Mateo-Cid y Mendoza-González, 1991; Ortega et al., 2001).
Catenella impudica (Montagne) J. Agardh.

Distribución: Campeche: litoral (Mateo-Cid et al., 2013b);

Quintana Roo: litoral (Cetz-Navarro et al., 2008).

Cystocloniaceae

Hypnea cervicornis J. Agardh.

Distribución: Veracruz: litoral (Sánchez-Rodríguez, 1980; De la Garza-Flores, 2003); Tabasco: litoral (Ramírez-López, 1996; Quiroz-González et al., 2018); Campeche: estuario (Ortega, 1995); Quintana Roo: estuario, litoral, insular (Mateo-Cid y Mendoza-González, 1991; Mendoza-González y Mateo-Cid, 1992; Collado-Vides y González-González, 1995).

Hypnea cornuta (Kützing) J. Agardh.

Distribución: Veracruz: insular (Godínez-Ortega et al., 2009); Tabasco: litoral (Mendoza-González et al., 2017); Campeche: litoral (Mateo-Cid et al., 2013b).

Hypnea musciformis (Wulfen) J.V. Lamouroux.

Distribución: Tamaulipas: estuario, litoral (Ortega et al., 2001; Vilchis et al., 2018); Veracruz: estuario, litoral, insular (Sánchez-Rodríguez, 1980; Ortega et al., 2001; De la Garza-Flores, 2003; González-Gándara et al., 2007; NúñezResendiz, 2009; Vilchis et al., 2018; De la Cruz-Francisco et al. 2017; Landa-Cansigno et al., 2019); Tabasco: litoral (Ramírez-López, 1996; Sentíes y Dreckmann, 2013; MendozaGonzález et al., 2017; Vilchis et al., 2018; Quiroz-González et al., 2018); Campeche: estuario, litoral (Ortega, 1995; Ortega et al., 2001; Mendoza-González et al., 2013; Mateo-Cid et al., 2013b; Vilchis et al., 2018; Núñez-Resendiz et al., 2019b); Yucatán: estuario, litoral, insular (Ortega et al., 2001; Ortegón-Aznar et al., 2001, 2009; Vilchis et al., 2018; Núñez-Resendiz et al., 2019a); Quintana Roo: litoral, insular (Mateo-Cid y Mendoza-González, 1991; MendozaGonzález y Mateo-Cid, 1992; Dreckmann et al., 1996; Ortega et al., 2001; Quan-Young et al., 2006; Mendoza-González et al., 2007). 
Hypnea spinella (C. Agardh) Kützing.

Distribución: Tamaulipas: estuario, litoral (Ortega et al., 2001); Veracruz: estuario, litoral, insular (Zizumbo AlamiIla, 1995; Mateo-Cid et al., 1996; Ortega et al., 2001; De la Garza-Flores, 2003; González-Gándara et al., 2007; NúñezResendiz, 2009; Galicia-García et al., 2013; De la Cruz-Francisco et al., 2017; Landa-Cansigno et al., 2019); Tabasco: estuario, litoral (Ramírez-López, 1996; Sentíes y Dreckmann, 2013; Mendoza-González et al., 2017; Quiroz-González et al., 2018); Campeche: estuario (Ortega, 1995; Ortega et al., 2001; Mateo-Cid et al., 2013b; Mendoza-González et al., 2013); Yucatán: estuario, litoral, insular (Ortega et al., 2001; Ortegón-Aznar et al., 2001, 2009); Quintana Roo: estuario, litoral, insular (Mateo-Cid y Mendoza-González, 1991; Mendoza-González y Mateo-Cid, 1992; Dreckmann et al., 1996; Ortega et al., 2001; Mateo-Cid et al., 2006; Quan-Young et al., 2006; ).

Hypnea valentiae (Turner) Montagne.

Distribución: Tamaulipas: litoral (Ortega et al., 2001); Veracruz: litoral, insular (Ortega et al., 2001; De la Garza-Flores, 2003; De la Cruz-Francisco et al., 2017; Luna-Ortega y De la Cruz-Francisco, 2017); Tabasco: estuario, litoral (MendozaGonzález et al., 2017; Quiroz-González et al., 2018); Campeche: estuario, litoral (Ortega, 1995; Ortega et al., 2001; Mateo-Cid et al., 2013b); Quintana Roo: litoral (Cetz-Navarro et al., 2008).

\section{Hypneocolax stellaris Børgesen.}

Distribución: Campeche: estuario (Ortega, 1995; Ortega et al., 2001; Mateo-Cid et al., 2013b); Quintana Roo: estuario, litoral, insular (Ortega et al., 2001).

\section{Dumontiaceae}

Dudresnaya crassa M. Howe.

Distribución: Veracruz: litoral, insular (Ortega et al., 2001; De la Garza-Flores, 2003; Vilchis et al., 2018); Quintana Roo: litoral, insular (Mateo-Cid et al., 2006; Mendoza-Gon- zález et al., 2007; Cetz-Navarro et al., 2008).

*Dudresnaya puertoricensis Searles \& Ballantine.

Distribución: Quintana Roo: insular (Mateo-Cid y Mendoza-González, 1991; Ortega et al., 2001).

\section{Gigartinaceae}

Chondracanthus acicularis (Roth) Fredericq.

Distribución: Veracruz: litoral, insular (Ortega et al., 2001; De la Garza-Flores, 2003); Tabasco: litoral (Mendoza-González et al., 2017); Quintana Roo: insular (Ortega et al., 2001).

*Chondracanthus elegans (Greville) Guiry.

= Gigartina elegans Greville.

Distribución: Quintana Roo: insular (Mateo-Cid y Mendoza-González, 1991).

Nota: reportada como Gigartina elegans para la región.

*Chondracanthus teedei (Mertens ex Roth) Kützing.

Distribución: Quintana Roo: litoral (Dreckmann et al., 1996; Ortega et al., 2001).

Kallymeniaceae

Austrokallymenia westii (Ganesan) C.W. Schneider \& G.W. Saunders.

इ Kallymenia westii Ganesan.

Distribución: Campeche: litoral (Mateo-Cid et al., 2013a, como Kallymenia westii); Quintana Roo: litoral, insular (Mateo-Cid et al., 2006; Cetz-Navarro et al., 2008, ambas como Kallymenia westii).

Kallymenia limminghei Montagne.

Distribución: Campeche: litoral (Callejas-Jiménez et al., 2005; Mateo-Cid et al., 2013a, b). 
Phyllophoraceae

Gymnogongrus crenulatus (Turner) J. Agardh.

Distribución: Campeche: litoral (Mateo-Cid et al., 2013b).

Gymnogongrus griffithsiae (Turner) C. Martius.

Distribución: Veracruz: estuario, litoral (Ortega et al., 2001; De la Garza-Flores, 2003); Tabasco: litoral (Mendoza-González et al., 2017); Campeche: estuario, litoral (Ortega et al., 2001; Mateo-Cid et al., 2013b); Quintana Roo: insular (Mateo-Cid y Mendoza-González, 1991; Ortega et al., 2001).

Gymnogongrus tenuis J. Agardh.

Distribución: Tamaulipas: litoral (Ortega et al., 2001); Veracruz: estuario, litoral (Sánchez-Rodríguez, 1980; Ortega et al., 2001; De la Garza-Flores, 2003; Núñes-Resendiz, 2009); Tabasco: litoral (Mendoza-González et al., 2017); Campeche: estuario, litoral (Ortega, 1995; Ortega et al., 2001; Mateo-Cid et al., 2013b); Quintana Roo: litoral, insular (Mateo-Cid y Mendoza-González, 1991; Ortega et al., 2001).

Rhizophyllidaceae

Contarinia magdae Weber Bosse.

Distribución: Campeche: litoral (Mateo-Cid et al., 2016); Yucatán: insular (Ortega et al., 2001).

Contarinia peyssonneliiformis Zanardini.

Distribución: Campeche: litoral (Mateo-Cid et al., 2016).

Ochtodes akumalensis Mateo-Cid, Mendoza-González \& Sentíes.

Distribución: Quintana Roo: litoral (Mateo-Cid et al., 2016).
Ochtodes maguachaveziae Mendoza- González, Mateo-Cid \& Sentíes.

Distribución: Quintana Roo: litoral (Mateo-Cid et al., 2016).

Ochtodes secundiramea (Montagne) M. Howe.

Distribución: Quintana Roo: litoral, insular (Mateo-Cid y Mendoza-González, 1991; Ortega et al., 2001; Mateo-Cid et al., 2006, 2016).

\section{Solieriaceae}

Agardhiella ramosissima (Harvey) Kylin.

Distribución: Veracruz: litoral (De la Garza-Flores, 2003); Campeche: estuario, litoral (Ortega, 1995; Ortega et al., 2001; Mateo-Cid et al., 2013b; Núñez-Resendiz et al., 2017a); Yucatán: litoral (Ortega et al., 2001; Núñez-Resendiz et al., 2017b); Quintana Roo: litoral, insular (Díaz-Martín y Quan-Young, 2001; Cetz-Navarro et al., 2008).

Agardhiella subulata (C. Agardh) Kraft \& M.J. Wynne.

Distribución: Tamaulipas: litoral (Ortega et al., 2001; Núñez-Resendiz et al., 2017a); Veracruz: litoral, insular (Ortega et al., 2001; De la Garza-Flores, 2003; Núñez-Resendiz et al., 2017a); Tabasco: litoral (Sentíes y Dreckmann, 2013; Núñez-Resendiz et al., 2017a; Quiroz-González et al., 2018); Campeche: estuario (Ortega, 1995; Ortega et al., 2001; Mateo-Cid et al., 2013b; Mendoza-González et al., 2013; Núñez-Resendiz et al., 2017a); Quintana Roo: litoral, insular (Díaz-Martín y Quan-Young, 2001; Ortega et al., 2001; Núñez-Resendiz et al., 2017a).

Eucheumatopsis isiformis (C. Agardh) Núñez-Resendiz, Dreckmann \& Sentíes.

इ Eucheuma isiforme (C. Agardh) J. Agardh.

Distribución: Veracruz: litoral, insular (Ortega et al., 2001; De la Garza-Flores, 2003; Núñez-Resendiz et al., 2017a); Tabasco: litoral (Sentíes y Dreckmann, 2013; Núñez-Re- 
sendiz et al., 2017a; Quiroz-González et al., 2018); Campeche: estuario, litoral (Ortega, 1995; Ortega et al., 2001; Callejas-Jiménez et al., 2005; Mateo-Cid et al., 2013b; Núñez-Resendiz et al., 2017a, 2019c); Yucatán: litoral (Ortega et al., 2001; Núñez-Resendiz et al., 2019b); Quintana Roo: litoral, insular (Mateo-Cid y Mendoza-González, 1991; Dreckmann et al., 1996; Ortega et al., 2001; Mendoza-González et al., 2007; Núñez-Resendiz et al., 2017a, 2019c).

Nota: esta especie ha sido ampliamente registrada en el AM como Eucheuma isiforme desde Mateo-Cid y MendozaGonzález (1991). Sin embargo, bajo evidencia molecular, esta especie fue transferida al género Eucheumatopsis Núñez-Resendiz, Dreckmann \& Sentíes bajo la nueva combinación Eucheumatopsis isiformis (Núñez-Resendiz et al. 2015, 2019b, c). Asimismo, tampoco existe evidencia genética para establecer la independencia de la variedad denudatum D.P. Cheney. De acuerdo con Núñez-Resendiz et al. (2019c), el género Euchema J. Agarth, no se encuentra en el Atlántico.

Flahaultia tegetiformans W.R. Taylor.

Distribución: Quintana Roo: litoral, insular (Dreckmann et al., 1996; Ortega et al., 2001; Mateo-Cid et al., 2006; Mendoza-González et al., 2007; Núñez-Resendiz et al., 2017a).

Kappaphycus inermis (F. Schmitz) Doty ex H.D. Nguyen \& Q.N. Huynh.

इ Eucheuma inerme F. Schmitz.

Distribución: Campeche: estuario (Ortega, 1995, como Eucheuma inerme; Ortega et al., 2001; Mateo-Cid et al., 2013b; Núñez-Resendiz et al., 2017a).

Meristotheca cylindrica M.L. Núñez-Resendiz, Dreckmann \& Sentíes.

Distribución: Campeche: litoral (Palma-Ortiz et al., 2017; Núñez-Resendiz et al., 2017a, 2019a).
Meristotheca gelidium (J. Agardh) E.J. Faye \& M. Masuda. इEucheuma gelidium (J.Agardh) J. Agardh.

= Eucheuma schrammii (P. Crouan \& H. Crouan).

= Meristiella echinocarpa (Areschoug) D.P. Cheney \& P.W. Gabrielson.

= Meristiella gelidium (J. Agardh) D.P. Cheney \& P.W. Gabrielson.

Distribución: Campeche: estuario, litoral (Ortega, 1995, como Eucheuma gelidium; Ortega et al., 2001, como Meristiella echinocarpa y Meristiella gelidium; CallejasJiménez et al., 2005; Mateo-Cid et al., 2013b, ambos como Meristiella gelidium; Mendoza-González et al., 2013; Núñez-Resendiz et al., 2017a); Yucatán: litoral (Ortega et al., 2001, como Meristiella echinocarpa y Meristiella gelidium; Núñez-Resendiz et al., 2017a); Quintana Roo: litoral, insular (Mateo-Cid y Mendoza-González, 1991; Mendoza-González y Mateo-Cid, 1992, ambas como Eucheuma gelidium; Dreckmann et al., 1996, como Meristiella echinocarpa y Meristiella gelidium; Díaz-Martín y Quan-Young, 2001, como Meristiella schrammii; Ortega et al., 2001, como Meristiella echinocarpa y Meristiella gelidium; Quan-Young et al., 2006, como Meristiella gelidium; Mendoza-González et al., 2007; Núñez-Resendiz et al., 2017a).

Nota: esta especie ha sido previamente registrada en la Península de Yucatán desde Ortega et al. (2001). Sin embargo, de acuerdo con Núñez-Resendiz et al. (2017a, 2019c), este nombre pudo ser erróneamente aplicado y en realidad lo que se ha registrado en la zona son las especies cilíndricas M. cylindrica Núñez-Resendiz, Dreckmann \& Sentíes o M. spinella Núñez-Resendiz, Dreckmann \& Sentíes, recientemente descritas bajo evidencia molecular para la Península de Yucatán. La presencia de M. gelidium no ha podido ser corroborada en la actualidad (Núñez-Resendiz et al., 2017a, 2019b).

Meristotheca spinella Núñez-Resendiz, Dreckmann \& Sentíes.

Distribución: Campeche: litoral (Núñez-Resendiz et al., 2019a). 
Tepoztequiella rhizoidea Núñez-Resendiz, Dreckmann \& Sentíes.

Distribución: Campeche: litoral (Núñez-Resendiz et al., 2017b); Quintana Roo: litoral (Núñez-Resendiz et al., 2017b).

Sarcodiotheca divaricata W.R. Taylor.

Distribución: Campeche: litoral (Mateo-Cid et al., 2013a).

Solieria filiformis (Kützing) P.W. Gabrielson.

Distribución: Tamaulipas: litoral (Ortega et al., 2001; NúñezResendiz et al., 2017a); Veracruz: litoral, insular (Ortega et al., 2001; De la Garza-Flores, 2003; Núñez-Resendiz et al., 2017a); Campeche: litoral (Ortega et al., 2001; Mateo-Cid et al., 2013a, b; Núñez-Resendiz et al., 2017a); Yucatán: insular (Ortega et al., 2001; Núñez-Resendiz et al., 2017a); Quintana Roo: insular (Mendoza-González y Mateo-Cid, 1992; Ortega et al., 2001; Mendoza-González et al., 2007; Núñez-Resendiz et al., 2017a).

Wurdemannia miniata (Sprengel) Feldmann \& Hamel.

Distribución: Veracruz: litoral, insular (Sánchez-Rodríguez, 1980; Ortega et al., 2001; De la Garza-Flores, 2003; NúñezResendiz et al., 2017a); Quintana Roo: litoral, insular (Mateo-Cid y Mendoza-González, 1991; Dreckmann et al., 1996; Ortega et al., 2001, Núñez-Resendiz et al., 2017a).

\section{Gracilariales}

\section{Gracilariaceae}

Crassiphycus caudatus (J. Agardh) Gurgel, J.N. Norris \& Fredericq.

E Gracilaria caudata J. Agardh.

इ Hydropuntia caudata (J. Agardh) Gurgel \& Fredericq.

Distribución: Tamaulipas: estuario, litoral (Ortega et al., 2001; Dreckmann, 2012, ambas como Gracilaria caudata; Dreckmann y Sentíes, 2013, 2014; Vilchis et al., 2018, como
Gracilaria caudata); Veracruz: estuario, litoral, insular (Dreckmann y De Lara-Isassi, 2000; Ortega et al., 2001; De la Garza-Flores, 2003; Dreckmann, 2012, reportada como Gracilaria caudata en las cuatro referencias anteriores; Dreckmann y Sentíes, 2013, 2014; De la Cruz-Francisco et al. 2017, ambas como Hydropuntia caudata; Vilchis et al., 2018, como Gracilaria caudata); Tabasco: estuario, litoral (Dreckmann y De Lara-Isassi, 2000; Dreckmann, 2012, ambas como Gracilaria caudata; Dreckmann y Sentíes, 2013, 2014, ambas como Hydropuntia caudata; Sentíes y Dreckmann, 2013; Mendoza-González et al., 2017; QuirozGonzález et al., 2018; Vilchis et al., 2018, reportada como Gracilaria caudata en las tres citas anteriores); Campeche: estuario, litoral (Ortega, 1995; Dreckmann y De Lara-Isassi, 2000; Ortega et al., 2001; Dreckmann, 2012, reportada como Gracilaria caudata en las cuatro citas anteriores; Dreckmann y Sentíes, 2013, 2014; Mateo-Cid et al., 2013b, ambas como Hydropuntia caudata; Vilchis et al., 2018); Quintana Roo: litoral, insular (Dreckmann, 2012, como Gracilaria caudata; Dreckmann y Sentíes, 2013, 2014, ambas como Hydropuntia caudata; Vilchis et al., 2018, como Gracilaria caudata).

Nota: a partir de evidencia molecular, las antiguas especies de Hydropuntia Montagne, registradas en el AM han sido transferidas al género Crassiphycus (Guiry et al., 2018; Gurgel et al., 2018).

Crassiphycus corneus (J. Agardh) Gurgel, J.N. Norris \& Fredericq.

三 Gracilaria cornea J. Agardh.

इ Hydropuntia cornea (J. Agardh) M.J. Wynne.

Distribución: Tamaulipas: litoral (Dreckmann, 2012, como Gracilaria cornea; Dreckmann y Sentíes, 2013, 2014; Vilchis et al., 2018, reportadas como Hydropuntia cornea en las tres referencias anteriores); Veracruz: litoral, insular (Ortega et al., 2001; De la Garza-Flores, 2003; Dreckmann, 2012, reportada como Gracilaria cornea en las tres referencias anteriores; Dreckmann y Sentíes, 2013, 2014; Vilchis et al., 2018, reportadas como Hydropuntia cornea en las tres citas anteriores); Campeche: estuario, litoral (Ortega et al., 2001, como Gracilaria cornea; Callejas-Jiménez et al., 2005, como 
Hydropuntia cornea; Dreckmann, 2012, como Gracilaria cornea; Dreckmann y Sentíes, 2013, 2014; Mateo-Cid et al., 2013b; Vilchis et al., 2018, reportadas como Hydropuntia cornea en las cuatro referencias anteriores; Núñez-Resendiz et al., 2015, 2019b, como Gracilaria cornea); Yucatán: litoral, insular (Ortega et al., 2001; Dreckmann, 2012, ambas como Gracilaria cornea; Dreckmann y Sentíes, 2013, 2014; Vilchis et al., 2018, reportadas como Hydropuntia cornea en las tres referencias anteriores; Núñez-Resendiz et al., 2019b, como Gracilaria cornea); Quintana Roo: litoral, insular (Dreckmann et al., 1996; Ortega et al., 2001, ambas como Gracilaria cornea; Quan-Young et al., 2006; MateoCid et al., 2006; Mendoza-González et al., 2007, reportadas como Hydropuntia cornea en las tres referencias anteriores; Dreckmann, 2012, como Gracilaria cornea; Dreckmann y Sentíes, 2013, 2014; Vilchis et al., 2018, reportadas como Hydropuntia cornea en las tres referencias anteriores; Núñez-Resendiz et al., 2015, como Hydropuntia cornea; Núñez-Resendiz et al., 2019b, como Gracilaria cornea).

Crassiphycus crassissimus (P. Crouan \& H. Crouan) Gurgel, J.N. Norris \& Fredericq.

三 Gracilaria crassissima (P. Crouan \& H. Crouan) P. Crouan \& H. Crouan.

$\equiv$ Hydropuntia crassissima (P. Crouan \& H. Crouan) M.J. Wynne.

Distribución: Yucatán: insular (Ortega et al., 2001); Quintana Roo: litoral, insular (Mateo-Cid y MendozaGonzález, 1991; Mendoza-González y Mateo-Cid, 1992; Dreckmann et al., 1996, reportado como Gracilaria crassissima en las tres citas anteriores; Ortega et al., 2001; Quan-Young et al., 2006; Mendoza-González et al., 2007, ambas como Hydropuntia crassissima; Dreckmann, 2012, como Gracilaria crassissima; Dreckmann y Sentíes, 2013, 2014, ambas como Hydropuntia crassissima).

Crassiphycus usneoides (C. Agardh) Gurgel, J.N. Norris \& Fredericq.

E Gracilaria usneoides (C. Agardh) J. Agardh.

इ Hydropuntia usneoides (C. Agardh) Gurgel \& Fredericq.

Distribución: Veracruz: litoral, insular (Dreckmann, 2012, como Gracilaria usneoides; Dreckmann y Sentíes, 2013, 2014, ambas como Hydropuntia usneoides); Campeche: estuario, litoral (Dreckmann, 2012, como Gracilaria usneoides; Dreckmann y Sentíes, 2013, 2014; Núñez-Resendiz et al., 2015, reportada como Hydropuntia usneoides en las tres referencias anteriores); Yucatán: litoral (Núñez-Resendiz et al., 2015, como Hydropuntia usneoides, Núñez-Resendiz et al., 2019b como Gracilaria usneoides); Quintana Roo: litoral, insular (Mateo-Cid y Mendoza-González, 1991; Ortega et al., 2001; Dreckmann, 2012, reportada como Gracilaria usneoides en las tres referencias anteriores; Dreckmann y Sentíes, 2013, 2014; Núñez-Resendiz et al., 2015, reportada como Hydropuntia usneoides en las tres referencias anteriores; Núñez-Resendiz et al., 2019b, como Gracilaria usneoides).

Gracilaria apiculata P. Crouan \& H. Crouan.

Distribución: Tamaulipas: estuario (Gurgel et al., 2003); Veracruz: litoral (Landa-Cansigno et al., 2019); Campeche: litoral (Gurgel et al., 2003).

Gracilaria armata (C. Agardh) Greville.

Distribución: Tamaulipas: estuario, litoral (Dreckmann y De Lara-Isassi, 2000; Ortega et al., 2001; Dreckmann, 2012); Veracruz: estuario, litoral (Dreckmann y De Lara-Isassi, 2000; Ortega et al., 2001; De la Garza-Flores, 2003; Dreckmann, 2012; Landa-Cansigno et al., 2019); Tabasco: estuario, litoral (Dreckmann y De Lara-Isassi, 2000); Campeche: litoral (Dreckmann y De Lara-Isassi, 2000; Ortega et al., 2001; Mateo-Cid et al., 2013b); Yucatán: litoral (Ortega et al., 2001).

\section{Gracilaria blodgettii Harvey.}

Distribución: Tamaulipas: litoral (Ortega et al., 2001; Dreckmann y Sentíes, 2009, 2013, 2014; Dreckmann, 2012; Vilchis et al., 2018); Veracruz: estuario, litoral, insular (Ortega et al., 2001; De la Garza-Flores, 2003; Dreckmann y Sentíes, 2009, 2013, 2014; Godínez-Ortega et al., 2009; Dreckmann, 2012; Vilchis et al., 2018; De la Cruz-Francisco et al., 2017); Tabasco: estuario, litoral (Ramírez-López, 1996; Dreckmann 
y Sentíes, 2009, 2013, 2014; Dreckmann, 2012; Sentíes y Dreckmann, 2013; Mendoza-González et al., 2017; Vilchis et al., 2018; Quiroz-González et al., 2018); Campeche: estuario, litoral (Ortega et al., 2001; Callejas-Jiménez et al., 2005; Dreckmann y Sentíes, 2009, 2013, 2014; Dreckmann, 2012; Mateo-Cid et al., 2013a, b; Mendoza-González et al., 2013; Vilchis et al., 2018; Núñez-Resendiz et al., 2019a); Yucatán: estuario, litoral, insular (Ortega et al., 2001; Ortegón-Aznar et al., 2001; Dreckmann y Sentíes, 2009, 2014; Dreckmann, 2012; Vilchis et al., 2018; Núñez-Resendiz et al., 2019b); Quintana Roo: litoral, insular (Mendoza-González y Mateo-Cid, 1992; Dreckmann et al., 1996; Ortega et al., 2001; Mateo-Cid et al., 2006; Quan-Young et al., 2006; Mendoza-González et al., 2007; Dreckmann y Sentíes, 2009, 2013, 2014; Dreckmann, 2012; Vilchis et al., 2018).

Gracilaria bursa-pastoris (S.G. Gmelin) P.C. Silva. = Gracilaria compressa (C. Agardh) Greville.

Distribución: Veracruz: estuario, litoral (Ortega et al., 2001; De la Garza-Flores, 2003; Dreckmann, 2012); Tabasco: litoral (Mendoza-González et al., 2017); Campeche: litoral (Mateo-Cid et al., 2013b); Quintana Roo: litoral, insular (Mateo-Cid y Mendoza-González, 1991; Ortega et al., 2001; Dreckmann, 2012, como Gracilaria compressa).

Gracilaria cervicornis (Turner) J. Agardh.

= Gracilaria ferox J. Agardh.

Distribución: Tamaulipas: litoral (Ortega et al., 2001; Vargas López et al., 2004; Dreckmann y Sentíes, 2009, 2013, 2014; Dreckmann, 2012; como Gracilaria ferox, Vilchis et al., 2018); Veracruz: estuario, litoral, insular (Sánchez-Rodríguez, 1980; Ortega et al., 2001; Dreckmann y Sentíes, 2009, 2013, 2014; Núñez-Resendiz, 2009; De la Garza-Flores, 2003; Dreckmann, 2012; como Gracilaria ferox, De la Cruz-Francisco et al. 2017; Vilchis et al., 2018); Tabasco: litoral (Ramírez-López, 1996; Dreckmann y Sentíes, 2009, 2013, 2014; Dreckmann, 2012, como Gracilaria ferox; Sentíes y Dreckmann, 2013; Mendoza-González et al., 2017; Quiroz-González et al., 2018; Vilchis et al., 2018); Campeche: estuario, litoral (Ortega, 1995; Ortega et al., 2001; Dreckmann y Sentíes, 2009, 2013, 2014; Dreckmann, 2012, como Gracilaria ferox; Mateo-Cid et al., 2013b; Vilchis et al., 2018; Núñez-Resendiz et al., 2019b); Yucatán: litoral (Ortega et al., 2001; Dreckmann y Sentíes, 2009, 2013, 2014; Dreckmann, 2012, como Gracilaria ferox; Vilchis et al., 2018); Quintana Roo: litoral, insular (Mateo-Cid y Mendoza-González, 1991; Mendoza-González y Mateo-Cid, 1992; Dreckmann et al., 1996; Ortega et al., 2001; Mendoza-González et al., 2007; Dreckmann y Sentíes, 2009, 2013, 2014; Dreckmann, 2012, como Gracilaria ferox; Vilchis et al., 2018; Núñez-Resendiz et al., 2019b).

\section{Gracilaria cuneata Areschoug.}

Distribución: Quintana Roo: insular (Mateo-Cid y Mendoza-González, 1991; Mendoza-González y Mateo-Cid, 1992; Ortega et al., 2001; Dreckmann, 2012; Vilchis et al., 2018).

\section{Gracilaria curtissiae J. Agardh.}

Distribución: Veracruz: litoral (Núñez-Resendiz, 2009); Campeche: estuario, litoral (Ortega, 1995; Ortega et al., 2001; Dreckmann, 2012).

Nota: Hardesty y Freshwater (2018) han sugerido que el registro de $G$. curtissiae en el Atlántico podría corresponder con un nombre erróneamente aplicado para $G$. mammillaris; sin embargo, el estatus de estos registros no ha sido evaluado aún para las costas del AM, por lo que se mantienen ambos registros por separado.

\section{Gracilaria cylindrica Børgesen.}

Distribución: Tamaulipas: litoral (Vargas López et al., 2004; Vilchis et al., 2018); Veracruz: litoral, insular (De la GarzaFlores, 2003; Núñez-Resendiz, 2009; De la Cruz-Francisco et al., 2017; Vilchis et al., 2018); Tabasco: estuario, litoral (Ramírez-López, 1996; Vilchis et al., 2018); Campeche: estuario (Ortega, 1995; Dreckmann, 2012; Mateo-Cid et al., 2013a, b; Vilchis et al., 2018); Yucatán: estuario, litoral, insular (Vilchis et al., 2018); Quintana Roo: litoral, insular (Mateo-Cid y Mendoza-González, 1991; Mendoza-González y Mateo-Cid, 1992; Dreckmann, 2012; Vilchis et al., 2018). 
Gracilaria damicornis J. Agardh.

Distribución: Veracruz: litoral, insular (Ortega et al., 2001; De la Garza-Flores, 2003; Dreckmann, 2012; Dreckmann y Sentíes, 2013, 2014; De la Cruz-Francisco et al., 2017); Tabasco: estuario, litoral (Ramírez-López, 1996; Dreckmann, 2012; Dreckmann y Sentíes, 2013, 2014; Sentíes y Dreckmann, 2013; Mendoza-González et al., 2017; QuirozGonzález et al., 2018); Campeche: estuario, litoral (Ortega, 1995; Ortega et al., 2001; Dreckmann, 2012; Dreckmann y Sentíes, 2013, 2014; Mateo-Cid et al., 2013b); Yucatán: estuario, litoral (Ortega et al., 2001; Ortegón-Aznar et al., 2001); Quintana Roo: litoral, insular (Mateo-Cid y Mendoza-González, 1991; Ortega et al., 2001; Dreckmann, 2012; Dreckmann y Sentíes, 2013, 2014).

Gracilaria debilis (Forsskål) Børgesen.

Distribución: Campeche: litoral (Mateo-Cid et al., 2013b); Yucatán: litoral (Dreckmann, 2012); Quintana Roo: litoral, insular (Dreckmann, 2012).

\section{*Gracilaria divaricata Harvey.}

Distribución: Tamaulipas: litoral (Ortega et al., 2001).

Gracilaria domingensis (Kützing) Sonder ex Dickie.

Distribución: Tamaulipas: litoral (Ortega et al., 2001; Vargas López et al., 2004; Dreckmann, 2012); Veracruz: estuario, litoral (Sánchez-Rodríguez, 1980; Ortega et al., 2001; De la Garza-Flores, 2003; Godínez-Ortega et al., 2009; Núñez-Resendiz, 2009; Dreckmann, 2012); Campeche: estuario, litoral (Ortega, 1995; Mateo-Cid et al., 2013b); Yucatán: litoral (Ortega et al., 2001); Quintana Roo: litoral, insular (Mateo-Cid y Mendoza-González, 1991; DíazMartín y Quan-Young, 2001; Ortega et al., 2001; Dreckmann, 2012).

Gracilaria flabelliformis (P. Crouan \& H. Crouan) Fredericq \& Gurgel.

Distribución: Veracruz: litoral (Landa-Cansigno et al., 2019);
Tabasco: litoral (Mendoza-González et al., 2017); Campeche: litoral (Núñez-Resendiz et al., 2019b).

\section{Gracilaria foliifera (Forsskål) Børgesen.}

Distribución: Tamaulipas: estuario, litoral (Ortega et al., 2001; Dreckmann, 2012); Veracruz: litoral, insular (Ortega et al., 2001; De la Garza-Flores, 2003; Godínez-Ortega et al., 2009; Dreckmann, 2012; De la Cruz-Francisco et al., 2017); Tabasco: litoral (Ramírez-López, 1996; Sentíes y Dreckmann, 2013; Quiroz-González et al., 2018); Campeche: estuario, litoral (Ortega et al., 2001; Dreckmann, 2012; Mateo-Cid et al., 2013b); Yucatán: litoral (Ortega et al., 2001); Quintana Roo: litoral, insular (Mateo-Cid y Mendoza-González, 1991; Ortega et al., 2001; Dreckmann, 2012).

Gracilaria gracilis (Stackhouse) Steentoft, L.M. Irvine \& Farnham.

Distribución: Tamaulipas: litoral (Ortega et al., 2001); Veracruz: estuario, litoral, insular (Ortega et al., 2001; De la Garza-Flores, 2003; Núñez-Resendiz, 2009); Campeche: estuario, litoral (Ortega et al., 2001; Mateo-Cid et al., 2013b); Yucatán: litoral, insular (Ortega et al., 2001); Quintana Roo: litoral, insular (Ortega et al., 2001).

Gracilaria isabellana Gurgel, Fredericq \& J.N. Norris.

= Gracilaria flabelliformis (P. Crouan \& H. Crouan) Fredericq \& Gurgel).

इ Gracilaria lacinulata (M. Vahl) M. Howe, nom. illeg. (non Gracilaria lacinulata (Kutzing) Piccone).

Distribución: Veracruz: litoral (Ortega et al., 2001; De la Garza-Flores, 2003, ambos como Gracilaria lacinulata).

Gracilaria mammillaris (Montagne) M. Howe.

Distribución: Tamaulipas: litoral (Ortega et al., 2001; Dreckmann y Sentíes, 2009, 2013, 2014; Dreckmann, 2012; Veracruz: litoral (Dreckmann y Sentíes, 2009, 2013, 2014; Dreckmann, 2012; De la Cruz-Francisco et al., 2017); Campeche: litoral (Callejas-Jiménez et al., 2005; 
Dreckmann y Sentíes, 2009, 2013, 2014; Dreckmann, 2012; Mateo-Cid et al., 2013a, b; Núñez-Resendiz et al., 2019b); Yucatán: litoral (Dreckmann y Sentíes, 2009); Quintana Roo: litoral, insular (Mateo-Cid y Mendoza-González, 1991; Mendoza-González y Mateo-Cid, 1992; Ortega et al., 2001; Mendoza-González et al., 2007; Dreckmann y Sentíes, 2009, 2013, 2014; Dreckmann, 2012; NúñezResendiz et al., 2019b).

Gracilaria microcarpa Dreckmann, Núñez-Resendiz \& Sentíes.

Distribución: Campeche: litoral (Núñez-Resendiz et al., 2019b).

Gracilaria multipartita (Clemente) Harvey.

Distribución: Tamaulipas: estuario (Dreckmann, 2012); Quintana Roo: litoral (Dreckmann et al., 1996).

Gracilaria ornata Areschoug.

Distribución: Quintana Roo: insular (Ortega et al., 2001; Dreckmann, 2012).

Gracilaria occidentalis (Børgesen) M. Bodard.

三 Rhodymenia occidentalis Børgesen.

Distribución: Veracruz: estuario, litoral (Ortega et al., 2001; De la Garza-Flores, 2003, ambas como Rhodymenia occidentalis).

Gracilaria pacifica I.A. Abbott.

Distribución: Tabasco: litoral (Quiroz-González et al., 2018).

Gracilaria tikvahiae Mc Lachlan.

Distribución: Tamaulipas: estuario, litoral (Ortega et al., 2001; Vargas López et al., 2004; Dreckmann y Sentíes, 2009, 2013, 2014; Dreckmann, 2012); Veracruz: litoral (Dreckmann y Sentíes, 2009, 2013, 2014; Dreckmann, 2012); Tabasco: litoral (Dreckmann y Sentíes, 2009, 2013, 2014;
Dreckmann, 2012; Quiroz-González et al., 2018); Campeche: estuario, litoral (Ortega, 1995), (Ortega et al., 2001; Dreckmann y Sentíes, 2009, 2013, 2014; Dreckmann, 2012; Mateo-Cid et al., 2013b; Núñez-Resendiz et al., 2019b); Yucatán: litoral (Dreckmann y Sentíes, 2009, 2013, 2014; Dreckmann, 2012; Núñez-Resendiz et al., 2019b); Quintana Roo: litoral (Dreckmann y Sentíes, 2009, 2013, 2014; Dreckmann, 2012).

\section{Gracilaria venezuelensis W.R. Taylor.}

Distribución: Veracruz: estuario, litoral (Ortega et al., 2001; De la Garza-Flores, 2003; Dreckmann, 2012); Tabasco: litoral (Quiroz-González et al., 2018); Campeche: estuario (Ortega, 1995; Ortega et al., 2001; Dreckmann, 2012; MateoCid et al., 2013b).

\section{Gracilaria wrightii (Turner) J. Agardh.}

Distribución: Quintana Roo: insular (Mateo-Cid y Mendoza-González, 1991; Mendoza-González y Mateo-Cid, 1992; Dreckmann, 2012).

Nota: Guiry y Guiry (2020) comentan que debido a la disparidad en las localidades de los sintipos de este taxón, no se puede asegurar la identidad de esta especie.

Gracilariopsis andersonii (Grunow) E.Y. Dawson.

Distribución: Campeche: litoral (Mateo-Cid et al., 2013b).

Nota: la distribución de esta especie se restringe al Pacífico de Baja California y California (Dawson, 1949), por lo que su presencia en Campeche debe ser revisada.

\section{Gracilariopsis costaricensis E.Y. Dawson.}

Distribución: Veracruz: litoral, insular (Ortega et al., 2001; De la Garza-Flores, 2003; Dreckmann y Sentíes, 2013, 2014).

Nota: de acuerdo con Hernández et al. (2020), esta especie es considerada un sinónimo de Gracilariopsis lemaneiformis del Pacífico americano. 
Gracilariopsis longissima (S.G. Gmelin) Steentoft, L.M. Irvine \& Farnham.

= Gracilaria verrucosa (Hudson) Papenfuss.

Distribución: Tamaulipas: litoral (Vargas López et al., 2004; Dreckmann, 2012, ambas como Gracilaria verrucosa); Veracruz: estuario, litoral, insular (Dreckmann, 2012, como Gracilaria verrucosa); Tabasco: litoral (Mendoza-González et al., 2017); Campeche: estuario (Ortega, 1995, como Gracilaria verrucosa; Dreckmann, 2012); Quintana Roo: insular (Mateo-Cid y Mendoza-González, 1991; MendozaGonzález y Mateo-Cid, 1992; Dreckmann, 2012, reportado como Gracilaria verrucosa en las tres referencias anteriores).

Gracilariopsis tenuifrons (C.J. Bird \& E.C. Oliveira) Fredericq \& Hommersand.

三 Gracilaria tenuifrons C.J. Bird \& E.C. Oliveira.

三 Gracilariopsis cata-luziana Gurgel, Fredericq \& J.N. Norris.

Distribución: Veracruz: litoral (Gurgel et al., 2003; Dreckmann y Sentíes, 2013, 2014, reportada como Gracilariopsis cata-luziana para la región); Tabasco: litoral (Ramírez-López, 1996, como Gracilaria tenuifrons; Dreckmann y Sentíes, 2013, 2014, ambas como Gracilariopsis cata-luziana; Sentíes y Dreckmann, 2013; Quiroz-González et al., 2018); Campeche: litoral (Dreckmann y Sentíes, 2013, 2014, ambas como Gracilariopsis cata-luziana); Quintana Roo: insular (Núñez-Resendiz et al., 2019b).

Nota: Gracilariopsis cata-luziana fue descrita originalmente de Veracruz, como una especie diferente de $G$. tenuifrons por presentar un diámetro menor en sus ramas (Gurgel et al., 2003). Sin embargo, de acuerdo a la evidencia molecular presentada por Hernández et al. (2020), G. cata-luziana fue reducida a sinonimia de $G$. tenuifrons.

Gracilariopsis sjoestedtii (Kylin) E.Y. Dawson Allan Hancock Found.

E Gracilaria sjoestedtii Kylin

Distribución: Veracruz: litoral (Sánchez-Rodríguez, 1980; Dreckmann, 2012); Quintana Roo: litoral (Dreckmann,
2012). Reportada en estas regiones como Gracilarias joestedtii.

Halymeniales

Halymeniaceae

Codiophyllum mexicanum Núñez-Resendiz, Dreckmann \& Sentíes.

Distribución: Campeche: litoral (Núñez-Resendiz et al., 2019c, 2020).

Corynomorpha clavata (Harvey) J. Agardh.

Distribución: Campeche: litoral (Mateo-Cid et al., 2013a); Quintana Roo: litoral (Ortega et al., 2001).

Cryptonemia crenulata (J. Agardh) J. Agardh.

Distribución: Campeche: litoral (Mateo-Cid et al., 2013a); Yucatán: insular (Ortega et al., 2001); Quintana Roo: litoral, insular (Mateo-Cid et al., 2006; Mendoza-González et al., 2007; Cetz-Navarro et al., 2008).

*Cryptonemia obovata J. Agardh.

Distribución: Tamaulipas: litoral (Ortega et al., 2001).

Cryptonemia seminervis (C. Agardh) J. Agardh.

Distribución: Campeche: litoral (Mateo-Cid et al., 2013a).

*Dermocorynus dichotomus (J. Agardh) Gargiulo, M. Morabito \& Manghisi.

इ Grateloupia dichotoma J. Agardh.

Distribución: Quintana Roo: insular (Ortega et al., 2001, como Grateloupia dichotoma).

Grateloupia filicina (J.V. Lamouroux) C. Agardh.

Distribución: Tamaulipas: litoral (Ortega et al., 2001; Vilchis 
et al., 2018); Veracruz: estuario, litoral, insular (SánchezRodríguez, 1980; Ortega et al., 2001; De la Garza-Flores, 2003; De la Cruz-Francisco et al., 2017; Vilchis et al., 2018); Tabasco: estuario, litoral (Ramírez-López, 1996; Sentíes y Dreckmann, 2013; Mendoza-González et al., 2017; QuirozGonzález et al., 2018); Campeche: estuario (Ortega et al., 2001; Mateo-Cid et al., 2013a; Vilchis et al., 2018); Yucatán: litoral (Vilchis et al., 2018); Quintana Roo: litoral, insular (Mateo-Cid y Mendoza-González, 1991; Mendoza-González y Mateo-Cid, 1992; Ortega et al., 2001).

\section{Grateloupia gibbesii Harvey.}

Distribución: Tabasco: litoral (Mendoza-González et al., 2017).

Grateloupia pterocladina (M.J. Wynne) S. Kawaguchi \& H.W. Wang.

$\equiv$ Prionitis pterocladina M.J. Wynne.

Distribución: Tamaulipas: litoral (Ortega et al., 2001); Tabasco: litoral (Mendoza-González et al., 2017, como Prionitis pterocladina).

Grateloupia subpectinata Holmes.

Distribución: Tabasco: litoral (Mendoza-González et al., 2017).

Halymenia duchassaingii (J. Agardh) Kylin.

Distribución: Veracruz: litoral, insular (Ortega et al., 2001; De la Garza-Flores, 2003); Quintana Roo: litoral (Ortega et al., 2001).

Halymenia echinophysa Collins \& M. Howe.

Distribución: Campeche: litoral (Mateo-Cid et al., 2013a).

Halymenia elongata C. Agardh.

Distribucion: Campeche: litoral (Mendoza-González et al., 2013); Yucatán: litoral (Ortegón-Aznar y Aguilar-Perera, 2014).
Halymenia floridana J. Agardh.

Distribución: Yucatán: estuario (Ortegón-Aznar et al., 2001); Quintana Roo: litoral, insular (Díaz-Martín y QuanYoung, 2001; Mendoza-González et al., 2007; Cetz-Navarro et al., 2008).

Halymenia hancockii W.R. Taylor.

Distribución: Veracruz: litoral (Godínez-Ortega et al., 2019).

Halymenia pseudofloresii Collins \& M. Howe.

Distribución: Tamaulipas: litoral (Ortega et al., 2001); Veracruz: estuario, litoral (Ortega et al., 2001; De la Garza-Flores, 2003); Tabasco: litoral (Sentíes y Dreckmann, 2013; Quiroz-González et al., 2018); Campeche: litoral (Mendoza-González et al., 2013; Núñez-Resendiz et al., 2019b; Callejas-Jiménez et al., 2005; Mateo-Cid et al., 2013a, b); Yucatán: estuario, litoral (Ortega et al., 2001; Ortegón-Aznar et al., 2001; Núñez-Resendiz et al., 2019b); Quintana Roo: litoral, insular (Díaz-Martín y Quan-Young, 2001; Cetz-Navarro et al., 2008; Mendoza-González y Mateo-Cid, 1992; Ortega et al., 2001; Mendoza-González et al., 2007).

Halymenia rosea M. Howe \& W.R. Taylor.

Distribución: Tamaulipas: litoral (Ortega et al., 2001); Quintana Roo: litoral, insular (Díaz-Martín y Quan-Young, 2001; Cetz-Navarro et al., 2008).

Hapalidiales

Hapalidiaceae

Melobesia membranacea (Esper) J.V. Lamouroux. Nouv.

Distribución: Veracruz: litoral, insular (Sánchez-Rodríguez, 1980; Ortega et al., 2001; De la Garza-Flores, 2003); Campeche: litoral (Ortega et al., 2001; Mateo-Cid et al., 2013b). 
Mesophyllaceae

Mesophyllum mesomorphum (Foslie) W.H. Adey.

Distribución: Veracruz: litoral, insular (Ortega et al., 2001; De la Garza-Flores, 2003); Quintana Roo: litoral, insular (Dreckmann et al., 1996; Ortega et al., 2001; Mateo-Cid et al., 2006).

Mesophyllum incertum (Foslie) Me. Lemoine.

Distribución: Campeche: litoral (Mateo-Cid et al., 2013a).

Mesophyllum ornatum (Foslie \& M. Howe) Athanasiadis.

Distribución: Campeche: litoral (Mateo-Cid et al., 2013a).

Hildenbrandiales

Hildenbrandiaceae

Hildenbrandia rubra (Sommerfelt) Meneghini.

= Hildenbrandia prototypus Nardo.

Distribución: Veracruz: litoral, insular (Sánchez-Rodríguez, 1980, como Hildenbrandia prototypus; Ortega et al., 2001; De la Garza-Flores, 2003); Tabasco: litoral (Mendoza-González et al., 2017).

Nemaliales

Galaxauraceae

Dichotomaria marginata (J. Ellis \& Solander) Lamarck. 三 Galaxaura marginata (Ellis \& Solander) J.V. Lamouroux.

Distribución: Veracruz: litoral, insular (Ortega et al., 2001; De la Garza-Flores, 2003, ambas como Galaxaura marginata; Luna-Ortega y De la Cruz-Francisco, 2017); Campeche: litoral (Mateo-Cid et al., 2013b); Quintana Roo: insular (Mendoza-González y Mateo-Cid, 1992; Ortega et al., 2001; Mateo-Cid et al., 2006, reportada como Galaxaura marginata en las tres referencias anteriores; MendozaGonzález et al., 2007).

Dichotomaria obtusata (J. Ellis \& Solander) Lamarck.

इ Galaxaura obtusata (J. Ellis \& Solander) J.V. Lamouroux.

Distribución: Veracruz: litoral, insular (Ortega et al., 2001; De la Garza-Flores, 2003, ambos como Galaxaura obtusata; Núñez-Resendiz, 2009; De la Cruz-Francisco et al., 2017); Campeche: litoral (Mateo-Cid et al., 2013a).

Galaxaura rugosa (J. Ellis \& Solander) J.V. Lamouroux.

= Galaxaura comans Kjellman.

= Galaxaura lapidescens (J. Ellis \& Solander) J.V. Lamouroux.

E Galaxaura subverticillata Kjellman.

Distribución: Veracruz: litoral, insular (Zizumbo Alamilla, 1995, como Galaxaura comans; Mateo-Cid et al., 1996; Ortega et al., 2001, como Galaxaura comans y Galaxaura subverticillata; De la Garza-Flores, 2003; GonzálezGándara et al., 2007; Galicia-García et al., 2013; De la CruzFrancisco et al., 2017; Luna-Ortega y De la Cruz-Francisco, 2017); Campeche: litoral (Ortega et al., 2001, como Galaxaura comans y Galaxaura subverticillata; Mateo-Cid et al., 2013a); Yucatán: insular (Ortega et al., 2001, como Galaxaura comans y Galaxaura subverticillata); Quintana Roo: litoral, insular (Mateo-Cid y Mendoza-González, 1991, como Galaxaura comans, Galaxaura subverticillata y Galaxaura lapidescens; Mendoza-González y Mateo-Cid, 1992; Dreckmann et al., 1996; Ortega et al., 2001, como Galaxaura comans y Galaxaura subverticillata en ambas; Mateo-Cid et al., 2006; Mendoza-González et al., 2007).

Tricleocarpa cylindrica (J. Ellis \& Solander) Huisman \& Borowitzka.

三 Galaxaura cylindrica (J. Ellis \& Solander) J.V. Lamouroux.

Distribución: Veracruz: litoral (Sánchez-Rodríguez, 1980, como Galaxaura cylindrica; Ortega et al., 2001; De la GarzaFlores, 2003; González-Gándara et al., 2007; Núñez-Resendiz, 2009); Yucatán: insular (Ortega et al., 2001); Quintana 
Roo: litoral, insular (Ortega et al., 2001; Mendoza-González et al., 2007).

Tricleocarpa fragilis (Linnaeus) Huisman \& R.A. Townsend. = Galaxaura oblongata (J. Ellis \& Solander) J.V. Lamouroux.

= Tricleocarpa oblongata (Ellis \& Solander) Huisman \& Borowitzka.

Distribución: Veracruz: litoral, insular (Sánchez-Rodríguez, 1980; Zizumbo Alamilla, 1995, como Galaxaura oblongata; Ortega et al., 2001; De la Garza-Flores, 2003; GonzálezGándara et al., 2007; Godínez-Ortega et al., 2009); Quintana Roo: litoral, insular (Mateo-Cid y Mendoza-González, 1991; Mendoza-González y Mateo-Cid, 1992, como Galaxaura oblongata; Dreckmann et al., 1996, como Tricleocarpa oblongata; Ortega et al., 2001; Mateo-Cid et al., 2006; Mendoza-González et al., 2007).

\section{Liagoraceae}

Ganonema farinosum (J.V. Lamouroux) K.C. Fan \& Yung C. Wang. = Liagora elongata Zanardini.

इ Liagora farinosa J.V. Lamouroux.

Distribución: Veracruz: litoral, insular (Huerta-Múzquiz, 1960, como Liagora elongata; Ortega et al., 2001; De la Garza-Flores, 2003); Campeche: litoral (Ortega et al., 2001); Yucatán: insular (Ortega et al., 2001); Quintana Roo: litoral, insular (Mateo-Cid y Mendoza-González, 1991; Dreckmann et al., 1996, ambas como Liagora farinosa; Ortega et al., 2001; Quan-Young et al., 2006; Mateo-Cid et al., 2006; Mendoza-González et al., 2007).

Ganonema megagynum (Børgesen) Huisman. 三 Liagora megagyna Børgesen.

Distribución: Veracruz: litoral, insular (Huerta-Múzquiz, 1960; Ortega et al., 2001; De la Garza-Flores, 2003); Yucatán: insular (Ortega et al., 2001); Quintana Roo: litoral, insular (Mateo-Cid y Mendoza-González, 1991; Ortega et al., 2001).

Nota: reportada en los tres estados como Liagora megagyna.
Ganonema pinnatum (Harvey) Huisman.

इ Liagora pinnata Harvey.

Distribución: Veracruz: litoral, insular (Ortega et al., 2001; De la Garza-Flores, 2003); Campeche: litoral (Ortega et al., 2001); Yucatán: insular (Ortega et al., 2001); Quintana Roo: insular (Mateo-Cid y Mendoza-González, 1991; Ortega et al., 2001; Mateo-Cid et al., 2006; Quan-Young et al., 2006).

Nota: reportada para estas regiones como Liagora pinnata.

Gloiocallis dendroidea (P. Crouan \& H. Crouan) Showe M. Lin, Huisman \& D.L. Ballantine.

इ Liagora dendroidea (P. Crouan \& H. Crouan) I.A. Abbott. = Liagora mucosa M. Howe

Distribución: Veracruz: litoral, insular (Ortega et al., 2001; De la Garza-Flores, 2003, ambas como Liagora dendroidea); Campeche: litoral (Ortega et al., 2001, como Liagora dendroidea); Yucatán: insular (Ortega et al., 2001, como Liagora dendroidea); Quintana Roo: litoral, insular (MateoCid y Mendoza-González, 1991; Dreckmann et al., 1996, ambas como Liagora mucosa; Ortega et al., 2001, como Liagora dendroidea).

*Helminthocladia calvadosii (J.V. Lamouroux ex Duby) Setchell.

Distribución: Quintana Roo: insular (Mateo-Cid y Mendoza-González, 1991; Ortega et al., 2001).

Liagora albicans J.V. Lamouroux.

Distribución: Yucatán: insular (Ortega et al., 2001); Quintana Roo: litoral, insular (Mateo-Cid et al., 2006; Cetz-Navarro et al., 2008).

Liagora ceranoides J.V. Lamouroux.

= Liagora ceranoides f. leprosa (J. Agardh) Yamada.

= Liagora pulverulenta $\mathrm{C}$. Agardh.

Distribución: Veracruz: litoral, insular (Sánchez-Rodríguez, 1980; Zizumbo Alamilla, 1995; Mateo-Cid et al., 1996; 
Ortega et al., 2001; De la Garza-Flores, 2003, ambas Liagora ceranoides f. leprosa; González-Gándara et al., 2007; Núñez-Resendiz, 2009; Galicia-García et al., 2013; Landa-Cansigno et al., 2019); Campeche: litoral (Ortega et al., 2001, como Liagora ceranoides f. leprosa; Mateo-Cid et al., 2013a); Yucatán: insular (Ortega et al., 2001, como Liagora ceranoides f. leprosa); Quintana Roo: litoral, insular (Mateo-Cid y Mendoza-González, 1991 como Liagora pulverulenta; Dreckmann et al., 1996; Díaz-Martín y QuanYoung, 2001; Ortega et al., 2001, como Liagora ceranoides f. leprosa; Mateo-Cid et al., 2006; Mendoza-González et al., 2007).

Liagora tsengii Huisman \& M.J. Wynne.

Distribución: Quintana Roo: insular (Mendoza-González y Mateo-Cid, 2007).

Liagora viscida (Forsskål) C. Agardh.

Distribución: Veracruz: litoral (Sánchez-Rodríguez, 1980; Ortega et al., 2001; De la Garza-Flores, 2003).

Titanophycus validus (Harvey) Huisman, G.W. Saunders \& A.R. Sherwood.

इ Liagora valida Harvey.

Distribución: Veracruz: litoral, insular (Huerta-Múzquiz, 1960; Sánchez-Rodríguez, 1980; Zizumbo Alamilla, 1995; Ortega et al., 2001, reportado como Liagora valida en las cuatro referencias anteriores; De la Garza-Flores, 2003; Galicia-García et al., 2013); Yucatán: insular (Ortega et al., 2001, como Liagora valida); Quintana Roo: litoral, insular (Mateo-Cid y Mendoza-González, 1991; Mendoza-González y Mateo-Cid, 1992; Dreckmann et al., 1996; Ortega et al., 2001; Mateo-Cid et al., 2006; reportada para toda la región como Liagora valida).

Trichogloea herveyi W.R. Taylor.

Distribución: Veracruz: litoral, insular (Mateo-Cid et al., 1996; Ortega et al., 2001; De la Garza-Flores, 2003).
Trichogloea requienii (Montagne) Kützing.

Distribución: Veracruz: litoral, insular (Ortega et al., 2001; De la Garza-Flores, 2003; Godínez-Ortega et al., 2009; Vilchis et al., 2018).

Trichogloeopsis pedicellata (M. Howe) I.A. Abbott \& Doty.

Distribución: Yucatán: insular (Ortega et al., 2001); Quintana Roo: litoral, insular (Mateo-Cid y MendozaGonzález, 1991; Dreckmann et al., 1996; Ortega et al., 2001; Díaz-Martín y Quan-Young, 2001; Mateo-Cid et al., 2006).

Liagoropsidaceae

Liagoropsis schrammii (P. Crouan \& H. Crouan) Doty \& I.A. Abbott.

इ Nemalion schrammii (P. Crouan \& H. Crouan) Børgesen.

Distribución: Veracruz: estuario, litoral (Huerta-Múzquiz, 1960, como Nemalion schrammii; Ortega et al., 2001; De la Garza-Flores, 2003).

Nemaliaceae

Nemalion elminthoides (Velley) Batters.

Distribución: Veracruz: litoral (Ortega et al., 2001; De la Garza-Flores, 2003).

Scinaiaceae

Scinaia complanata (Collins) A.D. Cotton.

Distribución: Tamaulipas: litoral (Ortega et al., 2001; Vargas López et al., 2004); Veracruz: litoral (Huerta-Múzquiz, 1960; Ortega et al., 2001; De la Garza-Flores, 2003); Campeche: litoral (Callejas-Jiménez et al., 2005; Mateo-Cid et al., 2013a, b); Quintana Roo: litoral, insular (Mateo-Cid et al., 2006; Mendoza-González et al., 2007; Cetz-Navarro et al., 2008). 
*Scinaia furcellata (Turner) J. Agardh.

Distribución: Tamaulipas: litoral (Ortega et al., 2001).

Nemastomatales

Nemastomataceae

Predaea feldmannii Børgesen.

Distribución: Veracruz: litoral, insular (Ortega et al., 2001; De la Garza-Flores, 2003); Campeche: litoral (Mateo-Cid et al., 2013a); Quintana Roo: litoral, insular (Mateo-Cid et al., 2006; Cetz-Navarro et al., 2008).

Schizymeniaceae

Platoma gelatinosum (M. Howe) C.W. Schneider, McDevit, G.W. Saunders \& C.E. Lane.

इ Nemastoma gelatinosum M. Howe.

Distribución: Campeche: litoral (Mateo-Cid et al., 2013b, como Nemastoma gelatinosum, Mateo-Cid et al., 2013a).

*Schizymenia ecuadoreana (W.R. Taylor) I.A. Abbott.

Distribución: Tamaulipas: litoral (Ortega et al., 2001).

Titanophora incrustans (J. Agardh) Børgesen.

Distribución: Campeche: litoral (Mateo-Cid et al., 2013b).

Peyssonneliales

Peyssonneliaceae

Peyssonnelia armorica (P. Crouan \& H. Crouan) Weber Bosse.

Distribución: Tabasco: litoral (Mendoza-González et al., 2017); Campeche: litoral (Mateo-Cid et al., 2013a); Quintana Roo: insular (Mendoza-González et al., 2007).
Peyssonnelia boergesenii Weber Bosse.

Distribución: Campeche: litoral (Mateo-Cid et al., 2013a); Quintana Roo: insular (Mateo-Cid y Mendoza-González, 1991; Ortega et al., 2001).

Peyssonnelia boudouresquei Yoneshigue.

Distribución: Quintana Roo: litoral, insular (Mateo-Cid et al., 2006; Cetz-Navarro et al., 2008).

Peyssonnelia conchicola Piccone \& Grunow.

Distribución: Campeche: litoral (Mateo-Cid et al., 2013a); Quintana Roo: litoral, insular (Mateo-Cid y Mendoza-González, 1991; Ortega et al., 2001; Mateo-Cid et al., 2006).

Peyssonnelia inamoena Pilger.

Distribución: Campeche: litoral (Mateo-Cid et al., 2013a); Quintana Roo: insular (Mateo-Cid et al., 2006; MendozaGonzález et al., 2007).

Peyssonnelia nordstedtii Weber Bosse.

Distribución: Yucatán: insular (Ortega et al., 2001); Quintana Roo: litoral, insular (Mateo-Cid et al., 2006; Cetz-Navarro et al., 2008).

Peyssonnelia rubra (Greville) J. Agardh.

Distribución: Veracruz: litoral (Núñez-Resendiz, 2009); Tabasco: litoral (Mendoza-González et al., 2017); Campeche: litoral (Mateo-Cid et al., 2013a); Quintana Roo: insular (Mateo-Cid y Mendoza-González, 1991).

Peyssonnelia simulans Weber Bosse.

Distribución: Quintana Roo: insular (Mateo-Cid y Mendoza-González, 1991; Mendoza-González y Mateo-Cid, 1992; Ortega et al., 2001; Mateo-Cid et al., 2006). 
Peyssonnelia stoechas Boudouresque \& Denizot.

Distribución: Quintana Roo: litoral, insular (Mateo-Cid et al., 2006; Cetz-Navarro et al., 2008).

Plocamiales

Plocamiaceae

Plocamium cartilagineum (Linnaeus) P.S. Dixon.

Distribución: Veracruz: litoral (De la Cruz-Francisco et al., 2017).

Rhodymeniales

Rhodymeniales incertaesedis

Agardhinula browneae (J. Agardh) De Toni.

Distribución: Campeche: litoral (Mateo-Cid et al., 2013a).

Champiaceae

*Champia minuscula A.B. Joly \& Ugadim.

Distribución: Quintana Roo: insular (Mateo-Cid y Mendoza-González, 1991; Ortega et al., 2001).

Champia parvula var. parvula (C. Agardh) Harvey.

Distribución: Tamaulipas: litoral (Ortega et al., 2001); Veracruz: estuario, litoral, insular (Sánchez-Rodríguez, 1980; Mateo-Cid et al., 1996; Ortega et al., 2001; De la GarzaFlores, 2003; Núñez-Resendiz, 2009); Campeche: estuario, litoral (Ortega, 1995; Ortega et al., 2001; Mateo-Cid et al., 2013a, b); Yucatán: estuario, litoral, insular (Ortega et al., 2001; Ortegón-Aznar et al., 2001, 2009); Quintana Roo: estuario, litoral, insular (Mateo-Cid y Mendoza-González, 1991; Mendoza-González y Mateo-Cid, 1992; Collado-Vides y González-González, 1995; Dreckmann et al., 1996; Ortega et al., 2001; Quan-Young et al., 2006; Mateo-Cid et al., 2006; Mendoza-González et al., 2007).
Champia parvula var. prostrata L.G. Williams.

Distribución: Veracruz: litoral, insular (Ortega et al., 2001; De la Garza-Flores, 2003); Quintana Roo: insular (MateoCid et al., 2006).

\section{Champia salicornioides Harvey.}

Distribución: Yucatán: insular (Ortega et al., 2001); Quintana Roo: litoral, insular (Mateo-Cid y MendozaGonzález, 1991; Mendoza-González y Mateo-Cid, 1992; Dreckmann et al., 1996; Ortega et al., 2001; Quan-Young et al., 2006; Mateo-Cid et al., 2006; Mendoza-González et al., 2007).

\section{Coelothrix irregularis (Harvey) Børgesen.}

Distribución: Veracruz: litoral, insular (Ortega et al., 2001; De la Garza-Flores, 2003; González-Gándara et al., 2007); Yucatán: insular (Ortega et al., 2001); Quintana Roo: litoral, insular (Mateo-Cid y Mendoza-González, 1991; MendozaGonzález y Mateo-Cid, 1992; Dreckmann et al., 1996; Ortega et al., 2001; Mateo-Cid et al., 2006).

*Gastroclonium parvum (Hollenberg) C.F. Chang \& B.M. Xia.

Distribución: Quintana Roo: litoral, insular (Dreckmann et al., 1996; Ortega et al., 2001).

Faucheaceae

Gloioderma atlanticum Searles.

三 Gloiocladia atlantica (Searles) R.E. Norris.

Distribución: Campeche: litoral (Mateo-Cid et al., 2013a); Quintana Roo: litoral, insular (Mateo-Cid et al., 2006; CetzNavarro et al., 2008, registrada para toda la región como Gloiocladia atlantica).

Gloiocladia blomquistii (Searles) R.E. Norris.

Distribución: Campeche: litoral (Mateo-Cid et al., 2013a). 
Gloiocladia rubrispora (Searles) R.E. Norris.

Distribución: Campeche: litoral (Mateo-Cid et al., 2013a).

Hymenocladiaceae

Asteromenia peltata (W.R. Taylor) Huisman \& A.J.K. Millar.

Distribución: Quintana Roo: insular (Mendoza-González y Mateo-Cid, 2007).

\section{Lomentariaceae}

Ceratodictyon intricatum (C. Agardh) R.E. Norris.

三 Gelidiopsis intricata (C. Agardh) Vickers.

Distribución: Veracruz: litoral, insular (Zizumbo Alamilla, 1995; Ortega et al., 2001, ambas como Gelidiopsis intricata; De la Garza-Flores, 2003; Galicia-García et al., 2013, como Gelidiopsis intricata); Campeche: litoral (Mendoza-González et al., 2013); Yucatán: insular (Ortega et al., 2001, como Gelidiopsis intricata); Quintana Roo: litoral, insular (MateoCid y Mendoza-González, 1991; Mendoza-González y Mateo-Cid, 1992; Ortega et al., 2001; Mateo-Cid et al., 2006, reportado como Gelidiopsis intricata para toda la región).

Ceratodictyon planicaule (W.R. Taylor) M.J. Wynne. 三 Gelidiopsis planicaulis (W.R. Taylor) W.R. Taylor.

Distribución: Tamaulipas: litoral (Ortega et al., 2001, como Gelidiopsis planicaulis); Vercaruz: litoral, insular (De la Garza-Flores, 2003, como Gelidiopsis planicaulis;); Campeche: litoral (Mendoza-González et al., 2013); Quintana Roo: insular (Mateo-Cid y Mendoza-González, 1991; Ortega et al., 2001; Mendoza-González et al., 2007, reportado como Gelidiopsis planicaulis para la región).

Ceratodictyon variable (J. Agardh) R.E. Norris.

= Gelidiopsis variabilis (Greville ex J. Agardh) F. Schmitz.

Distribución: Veracruz: litoral, insular (Mateo-Cid et al., 1996; Ortega et al., 2001; De la Garza-Flores, 2003, como
Gelidiopsis variabilis para esta región); Tabasco: litoral (Mendoza-González et al., 2017); Campeche: litoral (Mateo-Cid et al., 2013a, b; Mendoza-González et al., 2013).

Hooperia divaricata (Durant) M.J. Wynne, C.W. Schneider \& G.W. Saunders.

= Lomentaria baileyana (Harvey) Farlow.

इ Lomentaria divaricata (Durant) M.J. Wynne.

Distribución: Campeche: litoral (Ortega et al., 2001, como Lomentaria baileyana; Mateo-Cid et al., 2013b, como Lomentaria divaricata, Mateo-Cid et al., 2013b, como Lomentaria baileyana); Quintana Roo: litoral (Cetz-Navarro et al., 2008, como Lomentaria baileyana).

Lomentaria rawitscheri A.B. Joly.

Distribución: Campeche: litoral (Mendoza-González et al., 2013); Quintana Roo: litoral, insular (Mateo-Cid et al., 2006; Cetz-Navarro et al., 2008).

Lomentaria uncinata Martens ex Kützing.

Distribución: Veracruz: litoral (Ortega et al., 2001; De la Garza-Flores, 2003); Tabasco: litoral (Sentíes y Dreckmann, 2013; Quiroz-González et al., 2018).

Rhodymeniaceae

Botryocladia enteromorpha (Harvey) W.E. Schmidt, Lozada-Troche, D.L. Ballantine \& Fredericq.

इChrysymenia enteromorpha Harvey.

Distribución: Tabasco: litoral (Sentíes y Dreckmann, 2013; Quiroz-González et al., 2018); Campeche: litoral (Mateo-Cid et al., 2013a); Yucatán: insular (Ortega et al., 2001); Quintana Roo: litoral, insular (Mateo-Cid et al., 2006; Mendoza-González et al., 2007; Cetz-Navarro et al., 2008).

Nota: reportada como Chrysymenia enteromorpha para todas las regiones. 
Botryocladia occidentalis (Børgesen) Kylin.

Distribución: Tamaulipas: litoral (Ortega et al., 2001; Vargas López et al., 2004); Campeche: litoral (Ortega et al., 2001; Callejas-Jiménez et al., 2005; Mateo-Cid et al., 2013a, b; Núñez-Resendiz et al., 2019b); Quintana Roo: insular (Mendoza-González y Mateo-Cid, 1992; Ortega et al., 2001).

Botryocladia pyriformis (Børgesen) Kylin.

Distribución: Campeche: litoral (Mateo-Cid et al., 2013a); Quintana Roo: insular (Mateo-Cid y Mendoza-González, 1991; Mendoza-González y Mateo-Cid, 1992; Dreckmann et al., 1996; Ortega et al., 2001; Mateo-Cid et al., 2006; Quan-Young et al., 2006).

Botryocladia spinulifera W.R. Taylor \& I.A. Abbott.

Distribución: Campeche: litoral (Mateo-Cid et al., 2013a); Quintana Roo: litoral, insular (Mateo-Cid et al., 2006; Mendoza-González et al., 2007; Cetz-Navarro et al., 2008).

\section{Chrysymenia halymenioides Harvey.}

Distribución: Campeche: litoral (Mateo-Cid et al., 2013a); Quintana Roo: litoral, insular (Mateo-Cid et al., 2006; Mendoza-González et al., 2007; Cetz-Navarro et al., 2008).

*Chrysymenia planifrons (Melvill) J. Agardh.

Distribución: Quintana Roo: litoral (Ortega et al., 2001).

Chrysymenia ventricosa (J.V. Lamouroux) J. Agardh.

Distribución: Campeche: litoral (Mateo-Cid et al., 2013a).

Coelarthrum cliftonii (Harvey) Kylin.

Distribución: Quintana Roo: litoral, insular (Mateo-Cid et al., 2006; Cetz-Navarro et al., 2008).
*Cordylecladia peasiae Collins.

Distribución: Quintana Roo: insular (Mendoza-González y Mateo-Cid, 1992).

Rhodymenia pseudopalmata (J.V. Lamouroux) P.C. Silva.

Distribución: Tamaulipas: litoral (Ortega et al., 2001; Vargas López et al., 2004); Veracruz: litoral, insular (Ortega et al., 2001; De la Garza-Flores, 2003); Tabasco: litoral (QuirozGonzález et al., 2018); Campeche: litoral (Mateo-Cid et al., 2013a); Quintana Roo: insular (Mendoza-González y Mateo-Cid, 1992; Ortega et al., 2001).

\section{Rhodogorgonales}

Rhodogorgonaceae

Rhodogorgon ramosissima J.N. Norris \& Bucher.

Distribución: Veracruz: litoral, insular (Ortega et al., 2001; De la Garza-Flores, 2003; Vilchis et al., 2018).

Sebdeniales

Sebdeniaceae

Sebdenia flabellata (J. Agardh) P.G. Parkinson.

$\equiv$ Halymenia agardhii De Toni.

Distribución: Tamaulipas: litoral (Ortega et al., 2001); Veracruz: estuario, litoral (Ortega et al., 2001; De la GarzaFlores, 2003); Campeche: litoral (Mateo-Cid et al., 2013a, b); Quintana Roo: insular (Mendoza-González et al., 2007).

Erythropeltales

Erythrotrichiaceae

Erythrocladia irregularis Rosenvinge.

Distribución: Veracruz: litoral, insular (Sánchez-Rodríguez, 1980; Mateo-Cid et al., 1996; Ortega et al., 2001; De la Gar- 
za-Flores, 2003; Núñez-Resendiz, 2009); Campeche: litoral (Ortega, 1995; Ortega et al., 2001; Mateo-Cid et al., 2013b); Quintana Roo: insular (Mateo-Cid y Mendoza-González, 1991; Mendoza-González y Mateo-Cid, 1992; Ortega et al., 2001).

Erythrocladia pinnata W.R. Taylor.

Distribución: Veracruz: litoral (De la Garza-Flores, 2003); Yucatán: insular (Ortega et al., 2001).

Erythrotrichia carnea (Dillwyn) J. Agardh.

Distribución: Tamaulipas: litoral (Ortega et al., 2001); Veracruz: estuario, litoral, insular (Sánchez-Rodríguez, 1980; Zizumbo Alamilla, 1995; Ortega et al., 2001; De la GarzaFlores, 2003; Núñez-Resendiz, 2009); Tabasco: estuario, litoral (Ramírez-López, 1996; Sentíes y Dreckmann, 2013; Mendoza-González et al., 2017; Quiroz-González et al., 2018); Campeche: litoral (Ortega et al., 2001; Mateo-Cid et al., 2013b; Mendoza-González et al., 2013); Yucatán: litoral (Ortega et al., 2001); Quintana Roo: estuario, litoral, insular (Mateo-Cid y Mendoza-González, 1991; Collado-Vides y González-González, 1995; Dreckmann et al., 1996; Ortega et al., 2001; Mateo-Cid et al., 2006; Mendoza-González et al., 2007).

Erythrotrichia vexillaris (Montagne) Hamel.

Distribución: Campeche: litoral (Mateo-Cid et al., 2013b; Mendoza-González et al., 2016).

Sahlingia subintegra (Rosenvinge) Kornmann.

Distribución: Tamaulipas: litoral (Ortega et al., 2001); Veracruz: litoral, insular (Ortega et al., 2001; De la Garza-Flores, 2003); Tabasco: estuario, litoral (Mendoza-González et al., 2017; Quiroz-González et al., 2018); Campeche: litoral (Ortega et al., 2001; Mateo-Cid et al., 2013b; MendozaGonzález et al., 2016); Yucatán: insular (Ortega et al., 2001; Mendoza-González et al., 2016); Quintana Roo: estuario, litoral, insular (Ortega et al., 2001; Mendoza-González et al., 2016).
Rhodochaetales

Rhodochaetaceae

Rhodochaete pulchella Thuret ex Bornet.

Distribución: Quintana Roo: litoral (Cetz-Navarro et al., 2008; Mendoza-González et al., 2016).

Stylonematales

Stylonemataceae

*Bangiopsis dumontioides (P. Crouan \& H. Crouan) V. Krishnamurthy.

Distribución: Quintana Roo: litoral, insular (Ortega et al., 2001).

Chroodactylon ornatum (C. Agardh) Basson.

Distribución: Tamaulipas: estuario, litoral (Ortega et al., 2001); Veracruz: litoral, insular (Ortega et al., 2001; De la Garza-Flores, 2003; Godínez-Ortega et al., 2009); Tabasco: litoral (Mendoza-González et al., 2017); Campeche: litoral (Mateo-Cid et al., 2013b; Mendoza-González et al., 2016); Yucatán: insular (Ortega et al., 2001; Mendoza-González et al., 2016); Quintana Roo: litoral, insular (Mateo-Cid y Mendoza-González, 1991; Mendoza-González y Mateo-Cid, 1992; Ortega et al., 2001; Mendoza-González et al., 2007; 2016).

Stylonema alsidii (Zanardini) K.M. Drew. = Goniotrichum alsidii (Zanardini) M. Howe.

Distribución: Tamaulipas: litoral (Ortega et al., 2001); Veracruz: estuario, litoral, insular (Sánchez-Rodríguez, 1980, como Goniotrichum alsidii; Mateo-Cid et al., 1996; Ortega et al., 2001; De la Garza-Flores, 2003; Galicia-García et al., 2013); Tabasco: litoral (Mendoza-González et al., 2017); Campeche: litoral (Ortega, 1995; Ortega et al., 2001; Mateo-Cid et al., 2013b; Mendoza-González et al., 2013, 2016); Yucatán: insular (Ortega et al., 2001; Mendoza-Gon- 
zález et al., 2016); Quintana Roo: estuario, litoral, insular (Mateo-Cid y Mendoza-González, 1991; Mendoza-González y Mateo-Cid, 1992; Collado-Vides y González-González, 1995; Dreckmann et al., 1996; Ortega et al., 2001; MateoCid et al., 2006; Mendoza-González et al., 2007; MendozaGonzález et al., 2016).

\section{Discusión}

El presente listado reveló dos grandes cambios en la previamente conocida flora de las algas rojas. El primero de ellos cae en el terreno de la nomenclatura; es decir, sinonimias, nombres erróneamente aplicados, nuevas combinaciones y nuevas especies producto de la identificación molecularmente asistida; lo cual ha generado un cambio en la sistemática de los taxa (familias, géneros y especies). Por ejemplo, en el presente estudio detectamos 115 nombres de especies registradas para la zona, que actualmente corresponden con sinónimos, 26 para especies repotadas previo a Ortega et al. ( 2001) no contenidas en el catálogo y 114 para las registradas posteriormente a esta obra. El segundo tipo de cambio, que repercute en el número de especies, es el cambio de la flora en el tiempo. Un ejemplo ilustrativo es el mencionado por parte de Mateo-Cid et al. (2013b), de la ausencia de al menos 40 especies de rodofitas para Campeche, previamente registradas en trabajos desde 1966 al 2005, atribuyendo este hecho a los fenómenos de urbanización en el litoral del estado, o a la exclusión de algunas especies por el tipo y número de muestreos realizados. Sin embargo, en este trabajo se excluye la posibilidad de que la flora sea cambiante, no estática, a lo largo de medio siglo. Un ejemplo concreto de este tipo de cambio encontramos en Playa Paraíso, Morro de la Mancha, Veracruz, en la colección de macroalgas de UAMIZ. El último ejemplar recolectado de Gracilaria cervicornis (UAMIZ 482 a UAMIZ 489) fue en el año 1990, así como para Crassyphycus caudatus (UAMIZ 898), que tuvo su última recolecta en 1999; ningúna de estas especies ha sido registrada nuevamente a partir de estas fechas. Otros ejemplos de este patrón se encuentran resaltados en nuestro listado con un asterisco, o para el caso de Playa Paraíso/La Mancha, Veracruz (Núñez-Resendiz, 2009). En este sentido, en nuestro listado contamos 103 especies que fueron registradas después del 2001, más 54 registros previos al 2001 que han seguido siendo referidas en la zona antes y después del 2001. Desafortunadamente, la total ausencia de un análisis histórico detallado no nos permite establecer si este patrón de cambio es cíclico o permanente.

De las 451 especies registradas en este estudio, 22\% (101 spp.) únicamente se presentan en el Golfo de México, mientras que $25 \%$ (115 spp.) se distribuyen solo en el Caribe mexicano; 55\% (247 spp.) restante se distribuyen en ambas regiones, lo que sugiere un considerable intercambio florístico (Apéndice 2). Sin embargo, el porcentaje de especies exclusivas para cada región (aproximadamente $25 \%$ ) también revela un grado de aislamiento geográfico, principalmente del Golfo de México y particularmente de la Sonda de Campeche (Vilchis et al., 2018). Dicho aislamiento es consecuencia de que la corriente que pasa por el canal de Yucatán se desvía hacia el oeste bañando las costas del centro de Veracruz, sin tener influencia sobre el litoral de Campeche (Dreckmann y Sentíes, 2013; Vilchis et al., 2018). El comportamiento de dicha corriente, así como la composición florística que posee, ubica al litoral de Quintana Roo como la fuente que provee de especies al Golfo de México, particularmente a Veracruz y Tamaulipas, por lo que estos estados son florísticamente afines (Vilchis et al., 2018).

Dado que la heterogeneidad de hábitats de la costa de Quintana Roo es alta, también representa un ambiente más propicio para el establecimiento de comunidades algales, de las cuales las algas rojas representan el grupo dominante (348 spp.), en una proporción 3:1, con respecto al resto de los estados de la zona (Pedroche y Sentíes, 2003; Vilchis et al., 2018). De las familias reportadas aquí, las mas diversas fueron: Rhodomelaceae 21\% (96 spp.), Ceramiaceae 9\% (43 spp.), Delesseriaceae 7\% (34 spp.) y Gracilariaceae 7\% (34 spp.); representando 35\% del total de especies, solo en el estado. Sin embargo, algunas familias de importancia económica como Cystocloniaceae (6 spp., 1\%), Soleriaceae (12 spp., 3\%.) o Halymeniaceae (17 spp., 4\%), a pesar de ser poco diversas, son conspicuas en la región, tanto por su tamaño $(10$ a $100 \mathrm{~cm})$ como por su gran densidad poblacional.

Por otro lado, el hecho de que existan pocas familias y estados con un alto porcentaje de especies respecto 
al total reportado también puede ser consecuencia de un esfuerzo de muestreo altamente discriminante. Esto puede deberse a varias razones, fundamentalmente a la especialización por parte de los investigadores en el estudio de estas familias, y también a las características fisiográficas que no permiten una adecuada exploración, lo cual explicaría los pocos estudios y reportes para Tamaulipas (playas arenosas), Tabasco (sistemas estuarios) y Yucatán (aislamiento del litoral de las vías de comunicación). Adicionalmente a esto, también se suma la condición críptica ampliamente reportada en las algas de la región (NúñezResendiz et al., 2015, 2017a, 2019a, c; Dreckmann et al., 2018).

Finalmente, cabe destacar que las herramientas moleculares han permitido detectar la presencia de novedades taxonómicas, especialmente en la revelación de diversidad críptica (Núñez-Resendiz et al., 2015, 2017a, b, 2019a, c; Vilchis et al., 2018). Sin embargo, estas novedades taxonómicas o la resolución de complejos crípticos que han influido en el incremeno o reducción de la flora en la actualidad, bajo enfoques moleculares, se concentran en un reducido número de familias como Gracilariaceae (Núñez-Resendiz et al., 2015; Dreckmann et al., 2018), Rhodomelaceae (Sentíes et al., 2016) o Solieriaceae (Núñez-Resendiz et al., 2017a, c; 2019a, c), por lo que se requiere de una revisión más exhaustiva de la flora de algas rojas de la región bajo un enfoque integral y por un mayor número de especialistas. Consecuentemente, la incorporación de caractéres moleculares en los estudios de diversidad en un mayor número de familias, necesariamente redundarán en un incremento de la diversidad alfa. Dado lo anterior, resulta imperioso la necesidad de un monitoreo florístico constante a lo largo del litoral del Atlántico mexicano, principalmente por la gran cantidad de áreas protegidas en la región, un criterio propuesto, por primera vez en la ficología marina mexicana por Vilchis et al. (2018).

\section{Contribución de autores}

MLNR, KMD y AS concibieron y diseñaron el estudio. AMEGG y ECB realizaron la recopilación, los análisis e integración de la base de datos y resultados en general. Todos los autores contribuyeron con la redacción, discusión, revisión y aprobación del manuscrito final.

\section{Financiamiento}

Este estudio fue apoyado por los proyectos: UAMICBS2019-2022: sesión 15.18-281118 y UAMI-CA-117, Programa para el Desarrollo Profesional Docente de la Secretaría de Educación Pública (PRODEP-SEP).

\section{Agradecimientos}

A los revisores por su importante contribución para mejorar el manuscrito en su versión actual.

\section{Literatura Citada}

Boo, G. H., D. Robledo, G. Andrade-Sorcia y S. Min Boo. 2018. Genetic discontinuity of Digenea (Rhodomelaceae, Rhodophyta) from Mexico supports recognition of two new species, D. mexicana and D. rafaelii. Algae 33(3): 231-241. DOI: https://doi.org/10.4490/algae.2018.33.8.20

Callejas-Jiménez, M. E., A. Sentíes y K. M. Dreckmannn. 2005. Macroalgas bentónicas de Puerto Real, Faro de Santa Rosalía y Playa Preciosa, Campeche, México, con algunas consideraciones florísticas y ecológicas para el estado. Hidrobiológica 15(1): 89-96.

Cassano, V., M. C. Oliveira, M. C. Gil-Rodríguez, A. Sentíes, J. Díaz-Larrea y M. T. Fujii. 2012. Molecular support for the establishment of the new genus Laurenciella within the Laurencia complex (Ceramiales, Rhodophyta). Botanica Marina 55(4): 349-357. DOI: https://doi.org/10.1515/bot2012-0133

Cetz-Navarro, N. P., J. Espinoza-Avalos, A. Sentíes y L. I. QuanYoung. 2008. New records of macroalgae for the Mexican Atlantic and floristic richness of the Mexican Caribbean. Hidrobiológica 18(1): 11-19

Collado-Vides, L. y J. González-González. 1995. Patrones de distribución ficoflorístico en el sistema lagunar de Nichupté, Quintana Roo, México. Acta Botanica Mexicana 31: 19-32. DOI: https://doi.org/10.21829/abm31.1995.734

Collado-Vides, L., A. Duran, E. Armenis, V. Cassano, D. Burkepile, A. A. Shantz, L. Palma, J. Díaz-Larrea, A. Sentíes y M. T. Fujii. 2017. Seasonal recruitment and survival strategies of 
Palisada cervicornis comb. nov. (Ceramiales, Rhodophyta) in Coral Reefs Journal of Phycology 53: 1087-1096. DOI: https://doi.org/10.1111/jpy.12567

Dawson, E. Y. 1949. Studies of Northeast Pacific Gracilariaceae. Allan Hancock Foundation Publications. No. 7. The University California Press. Los Angeles, USA. Pp. 1-105.

De la Cruz-Francisco, V., R. E. Orduña-Medrano, J. E. ParedesFlores, R. I. Vázquez-Estrada, M. González-González y L. Flores-Galicia. 2017. Una aproximación a la florística y faunística de la costa rocosa El Pulpo, Cazones, Veracruz, México. CICIMAR Oceanides 32: 39-58.

De la Garza-Flores, C. 2003. Clave genérica de las algas rojas marinas macroscópicas y comunes de las costas de Veracruz, México. Tesis de licenciatura. Facultad de Estudios Superiores Iztacala, Universidad Nacional Autónoma de México. Cd Mx., México. 79 pp.

Díaz-Larrea, J., F. F. Pedroche y A. Sentíes. 2016. Impacto de los estudios moleculares en la ficología comparada mexicana: el caso de las macroalgas marinas. Cymbella 2(1): 1-15.

Díaz-Larrea, J., A. Sentíes, M. T. Fujii, F. F. Pedroche y M. Cabral Oliveira. 2007. Molecular evidence for Chondrophycus poiteaui var. gemmiferus comb. nov. (Ceramiales, Rhodophyta) from the Mexican Caribbean. Implications for the taxonomy of the Laurencia complex. Botanica Marina 50(4): 250-256. DOI: https://doi.org/10.1515/BOT.2007.026

Díaz-Martín, M. A. y L. I. Quan-Young. 2001. Ampliaciones de ámbito de 32 macroalgas de Isla Mujeres, Quintana Roo, México. Revista de Biología Tropical 49(1): 391-398.

Dreckmann, K. M. 1998. Clasificación y nomenclatura de las macroalgas marinas bentónicas del Atlántico Mexicano. Comisión Nacional para el Conocimiento y Uso de la Biodiversidad (CONABIO). Cd. Mx., México. 149 pp.

Dreckmann, K. M. 2012. Los géneros Gracilaria e Hydropuntia (Gracilariaceae, Rhodophyta) en las costas mexicanas del Golfo de México y Caribe. In: Sentíes, A. y K. M. Dreckmann (eds.). Monografías ficológicas, Vol. 4. Universidad Autónoma Metropolitana-Iztapalapa, México y Universidad de La Laguna, Tenerife. Cd. Mx., México. Pp. 111-204.

Dreckmann, K. M. y A. Sentíes. 1994. El alga Digenea simplex (Ceramiales: Rhodomelaceae) en México: variación biogeográfica. Revista de Biología Tropical 42(3): 443-45.

Dreckmann, K. M. y A. Sentíes. 2009. Gracilaria, subgenus Textoriella (Gracilariaceae, Rhodophyta) in the Gulf of
Mexico and the Mexican Caribbean. Revista Mexicana de Biodiversidad 80(3): 593-601. DOI: https://dx.doi. org/10.22201/ib.20078706e.2009.003.154

Dreckmann, K. M. y A. Sentíes. 2013. Las arribazones de algas marinas en el Caribe Mexicano, Evento biológico natural o basura en la playa. Biodiversitas 107: 7-11.

Dreckmann, K. M. y A. Sentíes. 2014. Biodiversidad de Gracilariaceae (Rhodophyta) en México. Revista Mexicana de Biodiversidad, Supl. 85: S69-S75. DOI: http://dx.doi. org/10.7550/rmb.40717

Dreckmann, K. M. y G. de Lara-Isassi. 2000. Gracilaria caudata J. Agardh (Gracilariaceae, Rhodophyta) en el Atlántico Mexicano. Hidrobiológica 10: 125-130.

Dreckmann, K. M. y M. A. Pérez-Hernández. 1994. Macroalgas bentónicas de la laguna de Tampamachoco, Veracruz, México. Revista de Biologia Tropical 42(3): 715-717.

Dreckmann, K. M., M. L. Núñez Resendiz y A. Sentíes. 2018. Gracilaria microcarpa sp. nov. (Gracilariaceae, Rhodophyta) from the southwestern Gulf of Mexico. Botanica Marina 61(2): 1-10. DOI: https://doi.org/10.1515/bot-2017-0068

Dreckmann, K. M., I. Stout y A. Sentíes. 1996. Lista actualizada de las algas marinas bentónicas de Puerto Morelos, Quintana Roo, Caribe Mexicano. Polibotánica 3: 1-17.

Galicia-García, C., N. M. Robinson y Y. B. Okolodkov. 2013. New records of red algae (Rhodophyta) for Cabezo reef, national park Sistema Arrecifal Veracruzano, Gulf of Mexico. Acta Botanica Mexicana 102: 39-76. DOI: https://doi. org/10.21829/abm101.2012.24

García-López, D. Y., L. E. Mateo-Cid y C. Mendoza-González. 2013. Estudio sobre el género Lithophyllum (Corallinales, Rhodophyta) en el Golfo de México y El Caribe Mexicano. Acta Botanica Venezuelica 36: 141-162.

García-Soto, G. y J. López-Bautista. 2018. Taxonomic notes on the genus Alsidium C. Agardh, including the merging of Bryothamnion Kützing (Rhodomelaceae). Algae 33(3): 215229. DOI: https://doi.org/10.4490/algae.2018.33.6.25

Godínez-Ortega, J. L., P. Ramírez-García y K. Pedraza Venegas. 2009. Cambios en la flora béntica de arrecife Hornos (Veracruz, México). Revista Especializada en Ciencias Químico-Biológicas 12: 59-65.

Godínez-Ortega, J. L., P. Ramírez-García, A. Granados-Barba y M. J. Wynne. 2019. New records of subtidal benthic marine algae from the state of Veracruz, southern Gulf of Mexico. 
Revista Mexicana de Biodiversidad 90: 1-17. DOI: https:// doi.org/10.22201/ib.20078706e.2019.90.2719

González-Gándara, C., M. Cruz Arellano, C. Domínguez Barradas, A. Serrano Solis y A. de J. Basañez Muñoz. 2007. Macroalgas asociadas a cuatro hábitats del arrecife Tuxpan, Veracruz, México. Revista UDO Agrícola 7: 252-257.

Guiry, M. D. y G. M. Guiry. 2020. AlgaeBase. World-wide electronic publication, National University of Ireland, Galway. https:// www.algaebase.org (consultado enero de 2020).

Guiry, M. D., J. N. Norris, S. Fredericq y C. F. D. Gurgel. 2018. Crassiphycus Guiry, Gurgel, J.N. Norris \& Fredericq, gen. nov., a replacement name for Crassa Gurgel, J.N. Norris \& Fredericq, nom. inval. (Gracilariaceae, Rhodophyta), with some additional nomenclatural notes. Notulae Algarum 82: 1-4

Gurgel, C. F. D., S. Fredericq y J. N. Norris. 2003. Gracilariopsis silvana sp. nov., G. hommersandii sp. nov. and G. cataluziana sp. nov. Three new species of Gracilariaceae (Gracilariales, Rhodophyta) from the western Atlantic. Hidrobiológica 13: 57-68.

Gurgel, C. F. D., J. N. Norris, W. E. Schmidt, H. N. Le y S. Fredericq. 2018. Systematics of the Gracilariales (Rhodophyta) including new subfamilies, tribes, subgenera, and two new genera, Agarophyton gen. nov. and Crassa gen. nov. Phytotaxa 374(1): 1-23. DOI: https://doi.org/10.11646/ phytotaxa.374.1.1

Hardesty, D. M. y D. W. Freshwater. 2018. Studies of North Carolina marine algae XIV: increased diversity of flattened offshore Gracilaria (Gracilariales, Rhodophyta) species revealed by DNA sequences of contemporary specimens and the G. mammillaris holotype. Botanica Marina 61(4): 407-413. DOI: https://doi.org/10.1515/bot-2017-0120

Hernández, O. E., K. M. Dreckmann, M. L. Núñez-Resendiz, M. I. Vilchis y A. Sentíes. 2020. Gracilariopsis lemaneiformis (Gracilariaceae, Rhodophyta) in the Mexican Coasts: A case of disjunct distribution? American Journal of Plant Sciences 11(2): 111-124. DOI: https://doi.org/10.4236/ ajps.2020.112009

Hernández, O. E., A. Sentíes, K. M. Dreckmann, V. Cassano y M. T. Fujii. 2017. Species diversity and biogeographical patterns of Laurencia sensu stricto (Rhodophyta) in the Atlantic Ocean. Hidrobiológica 27(3): 301-314. DOI: https://doi. org/10.24275/uam/izt/dcbs/hidro/2017v27n3/Hernadez
Huerta-Muzquiz, L. 1960. Lista preliminar de las algas marinas del litoral del estado de Veracruz. Boletín de la Sociedad Botánica de México 25: 39-45. DOI: https://doi. org/10.17129/botsci.1064

Landa-Cansigno, C., L. E. Mateo-Cid y J. A. Guerrero-Analco. 2019. Macroalgas marinas del litoral rocoso Neovolcánico de Veracruz, México. Acta Botanica Mexicana 126: e1525. DOI: https://doi.org/10.21829/abm126.2019.1525

López-Valdez, M. L. 2016. Biogeografía de Caulerpa (Chlorophyta) en México. Tesis de Maestría en Biología. Universidad Autónoma Metropolitana-Iztapalapa. Cd. Mx., México. 98 pp.

Luna-Ortega, I y V. De la Cruz-Francisco. 2017. Macroalgal assemblages on dead surfaces of scleractinian corals (Anthozoa: Scleractinia) in the Oro Verde reef, Veracruz, Mexico. CICIMAR Oceanides 32: 11-23.

Mateo-Cid, L. E. y A. C. Mendoza-González. 1991. Algas marinas benticas de la Isla Cozumel, Quintana Roo, México. Acta Botanica Mexicana 16: 57-87. DOI: https://doi. org/10.21829/abm16.1991.626

Mateo-Cid, L. E. y A. C. Mendoza-González. 2009. Revision of the Mexican species of Pneophyllum Kutzing (Corallinales, Rhodophyta). Revista de Biología Marina y Oceanografía 44(3): 603-618. DOI: https://doi.org/10.4067/S071819572009000300008

Mateo-Cid, L. E., A. C. Mendoza González y S. Fredericq. 2013a. A checklist of subtidal seaweeds from Campeche banks, Mexico. Acta Botanica Venezuelica 36(2): 95- 108.

Mateo-Cid, L. E., A. C. Mendoza-González y P. W. Gabrielson. 2014a. Neogoniolithon (Corallinales, Rhodophyta) on the Atlantic coast of Mexico, including $N$. siankanensis sp. nov. Phytotaxa 190(1): 064-093. DOI: https://dx.doi. org/10.11646/phytotaxa.190.1.7

Mateo-Cid, L. E., A. C. Mendoza González y C. Galicia García. 1996. Algas marinas de Isla Verde, Veracruz, México. Acta Botanica Mexicana 36: 59-75. DOI: https://doi. org/10.21829/abm36.1996.762

Mateo-Cid, L. E., A. C. Mendoza-González y R. B. Searles. 2003. The Tribe Callithamnieae (Ceramiaceae, Rhodophyta) in the Atlantic Coast of Mexico. Hidrobiológica 13: 39-50.

Mateo-Cid, L. E., A. C. Mendoza-González y R. B. Searles. 2006. A checklist and seasonal account of the deepwater Rhodophyta around Cozumel Island on the Caribbean Coast of Mexico. Caribbean Journal of Science 42(1): 39-52. 
Mateo-Cid, L. E., A. C. Mendoza-González, A. G. Ávila-Ortiz y S. Díaz Martínez. 2013b. Algas marinas bentónicas del litoral de Campeche, México. Acta Botanica Mexicana 104: 53-92. DOI: https://doi.org/10.21829/abm104.2013.57

Mateo-Cid, L. E., A. C. Mendoza-González, J. M. Norris y D. Y. García. 2018. A taxonomic account of species in the tribe Spongoclonieae (Ceramiaceae, Ceramiales, Rhodophyta) reported from Atlantic and Pacific Mexico. Phytotaxa 340(3): 229-245. DOI: https://doi.org/10.11646/phytotaxa.340.3.3

Mateo-Cid, L. E., A. C. Mendoza-González, A. Sentíes, J. Díaz-Larrea y J. A. Acosta-Calderon. 2014b. Laurencia laurahuertana sp. nov. (Rhodomelaceae, Rhodophyta): An epiphytic species from the Mexican Caribbean. Phycological Research 62(2): 94-101. DOI: https://doi.org/10.1111/pre.12043

Mateo-Cid, L. E., A. C. Mendoza-González, A. Sentíes, J. DíazLarrea, D. Y. García-López y B. Martínez-Daranas. 2016. Description of two new species of Ochtodes (Rhodophyta: Gigartinales) from Caribbean Mexico on the basis of morphological and molecular evidence. Botanica Marina 59(2-3): 131-146. DOI: https://doi.org/10.1515/bot-20150069

Mendoza-González, A. C. y L. E. Mateo-Cid. 1992. Algas marinas bentónicas de Isla Mujeres, Quintana Roo, México. Acta Botanica Mexicana 19: 37-61. DOI: https://doi. org/10.21829/abm19.1992.646

Mendoza-González, A. C. y L. E. Mateo-Cid. 2007. Cinco nuevos registros de las algas rojas (Rhodophyta) para el Caribe Mexicano. Polibotánica 23: 101-119.

Mendoza-González, A. C., L. E. Mateo-Cid y D. Y. García-López. 2017a. Inventory of benthic marine and estuarine algae and Cyanobacteria for Tabasco, Mexico. Biota Neotropica 17(4): e20170379. DOI: https://dx.doi.org/10.1590/1676-0611BN-2017-0379

Mendoza-González, A. C., L. E. Mateo-Cid y P. H. López Garrido. 2013. Algas marinas bentónicas asociadas a pecios y otras estructuras submareales de Campeche. Acta Botanica Venezuelica. 36: 119-140.

Mendoza-González, A. C., L. E. Mateo-Cid y R. B. Searles. 2007. Yucatán seaweeds from the offshore waters of Isla Mujeres, Quintana Roo, Mexico. Botanica Marina 50(5-6): 280-287. DOI: https://doi.org/10.1515/BOT.2007.032

Mendoza-González, A. C., L. E. Mateo-Cid, J. A. AcostaCalderon, A. Vázquez-Rodríguez, C. M. Hernández-Casas y G. A. Garduño-Acosta. 2016. Marine seaweeds of the Yucatán Península: diversity, economic importance and conservation. In: Riosmena-Rodríguez, R. (ed.). Marine benthos: biology, ecosystem functions and environmental impact. Hauppauge. New York, USA. Pp. 39-8.

Núñez-Resendiz, M. L. 2009. Catálogo de rodofitas del litoral del Morro de la Mancha, Veracruz. Tesis de licenciatura. Facultad de Ciencias, Universidad Nacional Autónoma de México. Cd. Mx., México. 145 pp. http://132.248.9.195/ ptd2009/marzo/0641206/Index.html (consultado enero de 2020).

Núñez-Resendiz, M. L., K. M. Dreckmann y A. Sentíes. 2017a. Meristotheca cylindrica sp. nov. (Solieriaceae, Rhodophyta) from the southern Gulf of Mexico. Phycologia 56(4): 423429. DOI: https://doi.org/10.2216/16-116.1

Núñez-Resendiz, M. L., K. M. Dreckmann, A. Sentíes y H. León-Tejera. 2019a. Meristotheca spinella (Solieriaceae, Rhodophyta) a new cylindrical species from the southwestern Gulf of Mexico. Cryptogamie, Algologie 40(6): 63-72. DOI: https://doi.org/10.5252/cryptogamiealgologie2019v40a6

Núñez-Resendiz, M. L., K. M. Dreckmann, M. J. Wynne y A. Sentíes. 2020. Codiophyllum mexicanum sp. nov. (Halymeniaceae, Rhodophyta), first record of a stalked red alga associated with sponges in the Western Atlantic. Phycologia 59: 89-98. DOI: https://doi.org/10.1080/00318884.2019.1683318

Núñez-Resendiz, M. L., A. Sentíes, K. M. Dreckmann y H. LeónTejera. 2017b. Biodiversidad de Solieriaceae (Gigartinales, Rhodophyta) en México. Cymbella 3(2): 21-31.

Núñez-Resendiz, M. L., K. M. Dreckmann, A. Sentíes, M. J. Wynne y H. León-Tejera. 2019b. Marine red algae (Rodophyta) of economic use in the algal drifts from the Yucatán Peninsula, Mexico. Phytotaxa 387(3): 219-240. DOI: http://dx.doi. org/10.11646/phytotaxa.387.3.3

Núñez-Resendiz, M. L, K. M. Dreckmann, A. Sentíes, M. J. Wynne y H. León-Tejera. 2019c. Eucheumatopsis isiformis gen. \& comb. nov. (Solieriaceae, Rhodophyta) from the Yucatán Peninsula, to accommodate Eucheuma isiforme. Phycologia 58(1): 1-12. DOI: http://doi.org/10.1080/00318884.2018.1 517536

Núñez-Resendiz, M. L., K. M. Dreckmann, A. Sentíes, J. DíazLarrea y G. C. Zuccarello. 2015. Genetically recognizable but not morphologically: the cryptic nature of Hydropuntia 
cornea and $H$. usneoides (Gracilariales, Rhodophyta) in the Yucatán Peninsula. Phycologia 54(4): 407-416. DOI: https:// doi.org/10.2216/15-009.1

Núñez-Resendiz, M. L., K. M. Dreckmann, A. Sentíes, G. C. Zuccarello y H. León-Tejera. 2017c. Tepoztequiella rhizoidea gen. et sp. nov. (Solieriaceae, Rhodophyta) from the Yucatán Peninsula, Mexico. Phycologia 57(1): 90-99. DOI: https:// doi.org/10.2216/17-60.1

Orozco-Vega, H. y K. M. Dreckmann. 1995. Macroalgas estuarinas del litoral mexicano del Golfo de México. Cryptogamie, Algologie 16: 189-198.

Ortega, M. M. 1995. Observaciones del fitobentos de la Laguna de Términos, Campeche, México. Anales del Instituto de Biología, Universidad Nacional Autónoma de México, Serie Botánica 66: 1-36.

Ortega, M. M., J. L. Godínez y G. Garduño-Solórzano. 2001. Catálogo de algas bénticas de las costas mexicanas del Golfo de México y Mar Caribe. Instituto de Biología, Universidad Nacional Autónoma de México. Cd. Mx., México. 594 pp.

Ortegón-Aznar, I. y A. Aguilar-Perera. 2014. Distribución de las macroalgas en áreas naturales protegidas de la costa norte de la Península de Yucatán, México. Revista de Investigaciones Marinas 34(2): 1-12.

Ortegón-Aznar, I., J. González-González y A. Sentíes. 2001. Estudio ficoflorístico de la laguna de Río Lagartos, Yucatán, México. Hidrobiológica 11: 97-104.

Ortegón-Aznar, I., I. Sánchez-Molina y R. H. Casanova Cetz. 2009. The distribution of marine algae in a coastal lagoon, northern Yucatán Peninsula, Mexico. Neotropical Biology and Conservation 4(2): 99-105. DOI: https://doi. org/10.4013/nbc.2009.42.05

Palma-Ortiz, C. A., K. M. Dreckmann, M. L. Núñez Reséndiz y A. Sentíes. 2017. Variación genética en Meristotheca cylindrica (Solieriaceae, Rhodophyta) en Campeche, México. Hidrobiológica 27(3): 315-326. DOI: https://doi. org/10.24275/uam/izt/dcbi/hidro/2017v27n3/Palma

Pedroche, F. F. y A. Sentíes. 2003. Ficología marina mexicana: Diversidad y Problemática actual. Hidrobiológica 13(1): 23-32.

Pedroche, F. F., A. Sentíes, E. Novelo y E. Meave Del Castillo. 2009. Algas. In: Herrero, C. (ed.). Cosmos: Enciclopedia de las Ciencias y la Tecnología en México. Consejo Nacional de Ciencia y Tecnología/Universidad Autónoma Metropolitana. Cd. Mx., México. Pp. 54-69.
Quan-Young, L. I., M. A. Díaz-Martin y J. Espinoza-Avalos. 2006. Algas epífitas de Bajo Pepito, Isla Mujeres, Quintana Roo, México. Revista de Biología Tropical 54(2): 317-328. DOI: https://doi.org/10.15517/rbt.v54i2.13872

Quiroz-González, N., D. León-Álvarez y M. G. Rivas Acuña. 2018. Biodiversidad de las algas rojas marinas (Rhodophyta) en Tabasco, México. Acta Botanica Mexicana 123: 103-120. DOI: https://doi.org/10.21829/abm123.2018.1253

Ramírez-López, A. A. 1996. Estudio preliminar de las algas rojas (Rhodophyta) del litoral del estado de Tabasco, México. Tesis de licenciatura. Facultad de Estudios Superiores Campus Iztacala, Universidad Nacional Autónoma de México. Cd. Mx., México. 66 pp.

Sánchez-Rodríguez, M. E. 1980. Ficoflora del sustrato rocoso dentro de las costas del Golfo de México, México. Boletim do Instituto Paulista de Oceanografía 29(2): 347-350. DOI: http://dx.doi.org/10.1590/S0373-55241980000200069

Schneider, C. W., C. E. Lane y G. W. Saunders. 2010. Notes on the marine algae of the Bermudas. 11. More additions to the benthic flora and a phylogenetic assessment of Halymenia pseudofloresii (Halymeniales, Rhodophyta) from its type locality. Phycologia 49(2): 154-168. DOI: https://doi. org/10.2216/PH09-46.1

Schneider, C. W., C. E. Lane y G. W. Saunders. 2018. A revision of the genus Cryptonemia (Halymeniacea, Rhodophyta) in Bermuda, western Atlantic Ocean, including five new species and C. bermudensis (Collins \& M. Howe) comb. nov. European Journal of Phycology 53(3): 350-368. DOI: https:// doi.org/10.1080/09670262.2018.1452297

Sentíes, A. y J. Díaz-Larrea. 2008. Proposals for Palisada poiteaui var. gemmifera comb. nov. and Palisada corallopsis comb. nov. (Rhodomelaceae, Rhodophyta). Botanica Marina 51(1): 69-70. DOI: https://doi.org/10.1515/BOT.2008.004

Sentíes, A. y K. M. Dreckmann. 2011. Reino Vegetal. Diversidad de especies: Macroalgas. In: Villalobos Zapata, A. (ed.). La biodiversidad en Campeche: Estudio de Estado. Comisión Nacional para el Conocimiento y Uso de la Biodiversidad (CONABIO), Universidad Autónoma de Campeche, EI Colegio de la Frontera Sur (ECOSUR). Cd. Mx., México. pp. 202-207.

Sentíes, A. y K. M. Dreckmann. 2013. Lista actualizada de las macroalgas de Tabasco, México. Acta Botanica Venezuelica 36: 109-117. 
Sentíes, A. y K. M. Dreckmann. 2014. Biodiversidad de las macroalgas marinas de la familia Rhodomelaceae (Rhodophyta) en México. Revista Mexicana de Biodiversidad, Supl. 85: 62-68. DOI: https://doi.org/10.7550/rmb.40713

Sentíes, A. y M. T. Fujii. 2002. El complejo Laurencia (Rhodomelaceae, Rhodophyta) en el Caribe mexicano. In: Sentíes, A. y K. M. Dreckmann (eds.). Monografías Ficológicas. Universidad Autonoma Metropolitana Campus Iztapalapa, México y Red Latinoamericana de Botánica, Chile. Cd. Mx., México. Pp: 119-192.

Sentíes, A., M. T. Fujii y D. Rodríguez. 2001. Laurencia venusta (Ceramiales, Rhodophyta): a new record from the Atlantic Ocean. Botanica Marina 44(2): 95-99. DOI: https://doi. org/10.1515/ВOT.2001.013

Sentíes, A., J. Díaz-Larrea, V. Cassano, M. C. Gil-Rodríguez y M. T. Fujii. 2009. Palisada perforata (Rhodomelaceae, Ceramiales) en el Caribe mexicano. Revista Mexicana de Biodiversidad 80(001): 7-12. DOI: https://doi.org/10.22201/ ib.20078706e.2009.001.577

Sentíes, A., J. Díaz-Larrea, V. Cassano, M. C. Gil-Rodríguez y M. T. Fujii. 2011. Laurencia marilzae (Ceramiales, Rhodophyta) from the Mexican Caribbean: a new record for the tropical western Atlantic. Bulletin of Marine Science 87(3): 681-686. DOI: https://doi.org/10.5343/bms.2010.1094

Sentíes, A., V. Cassano, K. M. Dreckmann, M. C. Gil-Rodríguez, E. M. Stein y M. T. Fujii. 2016. Chondrophycus anabeliae (Rhodomelaceae, Ceramiales), a new species in the Laurencia complex from the Mexican Caribbean. Phytotaxa 283(3): 259-270. DOI: https://doi.org/10.11646/ phytotaxa.283.3.2

Sentíes, A., M. J. Wynne, V. Cassano, J. Díaz-Larrea, M. C. GilRodriguez y M. T. Fujii. 2015. Yuzurua iridescens (M.J. Wynne \& D.L. Ballantine) comb. nov. (Ceramiales, Rhodophyta) from the Caribbean Sea: morphological and molecular evidence. Brazilian Journal of Botany 38(3): 605-613. DOI: https://doi.org/10.1007/s40415-015-0154-x
Silva, P. C., P. W. Basson y R. L. Moe. 1996. Catalogue of the benthic marine algae of the Indian Ocean. University of California Publications in Botany 79: 1-1259 pp.

Tapia-Silva, F. O., O. E. Hernández-Cervantes, M. I Vilchis-Alfaro, A. Sentíes y K. M. Dreckmann. 2015. Mapping of algae richness using spatial data interpolation. International Archives Photogrammetry Remote Sensing and Spatial Information Sciences XL-7/W3: 1005-1008. DOI: https:// doi.org/10.5194/isprsarchives-XL-7-W3-1005-2015

Thiers, B. 2020. Index Herbariorum: a global directory of public herbaria and associated staff. New York Botanical Garden's. New York, USA. http://www.nybg.org/bsci/ih/ih.html (consultado noviembre de 2019).

Vargas López, V. R., S. Martínez Lozano, J. Verde-Star, H. GámezGonzález, R. Mercado, L. Villarreal-Rivera y M. GonzálezÁlvarez. 2004. Potencial económico de la flora ficológica del estado de Tamaulipas, México. Revista Internacional de Botánica Experimental фYTON 73: 171-179.

Vázquez-Delfín, E., Y. Freile-Pelegrín, H. Pliego-Cortés y D. Robledo. 2019. Seaweed resources of Mexico: current knowledge and future perspectives. Botanica Marina 62(3): 275-289. DOI: https://doi.org/10.1515/bot-2018-0070

Vilchis, M. I., K. M. Dreckmann, E. A. García-Trejo, O. E. Hernández y A. Sentíes. 2018. Patrones de distribución de las grandes macroalgas en el Golfo de México y el Caribe mexicano: una contribución a la biología de la conservación. Revista Mexicana de Biodiversidad 89(1): 183-192. DOI: https://doi. org/10.22201/ib.20078706e.2018.1.2226

Wynne, M. J. 2017. A checklist of benthic marine algae of the tropical and subtropical western Atlantic: fourth revision. Nova Hedwigia Beiheft 145: 7-202.

Zizumbo Alamilla, L. E. 1995. Estudio ficoflorístico de las macroalgas bénticas del arrecife coralino Isla Verde, Veracruz, México. Tesis de licenciatura. Facultad de Estudios Superiores Campus Iztacala, Universidad Nacional Autónoma de México. Cd. Mx., México. 113 pp. 
Apéndice 1. Publicaciones consultadas para la actualización florística por estado. Abreviaciones: Tamaulipas (T), Veracruz (V), Tabasco (Tb), Campeche (C), Yucatán (Y) y Quintana Roo (QRoo). Las publicaciones previas a 2001 referidas en esta tabla no fueron consideradas previamente en Ortega et al., 2001.

\begin{tabular}{|c|c|}
\hline Publicaciones & Estados \\
\hline Callejas-Jiménez et al., 2005 & $\mathrm{C}$ \\
\hline Cassano et al., 2012 & C, QRoo \\
\hline Cetz-Navarro et al., 2008 & QRoo \\
\hline Collado-Vides y González-González, 1995 & QRoo \\
\hline De la Cruz-Francisco et al., 2017 & $\mathrm{~V}$ \\
\hline De la Garza-Flores, 2003 & $\mathrm{~V}$ \\
\hline Díaz-Larrea et al., 2007 & QRoo \\
\hline Díaz-Martín y Quan-Young, 2001 & QRoo \\
\hline Dreckmann y Sentíes, 1994 & $T, V, C, Y, Q R o o$ \\
\hline Dreckmann et al., 1996 & QRoo, \\
\hline Dreckmann y De Lara-Isassi, 2000 & $\mathrm{~T}, \mathrm{~V}, \mathrm{~Tb}, \mathrm{C}$ \\
\hline Dreckmann y Sentíes, 2009 & T, V, Tb, C, Y, QRoo \\
\hline Dreckmann, 2012 & T, V, Tb, C, Y, QRoo \\
\hline Dreckmann y Sentíes, 2013 & T, V, Tb, C, Y, QRoo \\
\hline Dreckmann y Sentíes, 2014 & T, V, Tb, C, Y, QRoo \\
\hline Galicia-García et al., 2013 & V \\
\hline García-López et al., 2013 & V, C, Y, QRoo \\
\hline Godínez-Ortega et al., 2009 & V \\
\hline Godínez-Ortega et al., 2019 & V \\
\hline González-Gándara et al., 2007 & V \\
\hline Gurgel et al., 2003 & $\mathrm{~T}, \mathrm{~V}, \mathrm{C}$ \\
\hline Hernández et al., 2017 & $T, V, C, Y, Q R o o$ \\
\hline Landa-Cansigno et al., 2019 & V \\
\hline Luna-Ortega y De la Cruz-Francisco, 2017 & V \\
\hline Mateo-Cid y Mendoza-González, 1991 & QRoo \\
\hline Mateo-Cid et al., 1996 & $\mathrm{~V}$ \\
\hline Mateo-Cid et al., 2003 & T, V, Tb, C, Y, QRoo \\
\hline Mateo-Cid et al., 2006 & QRoo \\
\hline Mateo-Cid y Mendoza-González, 2009 & $T, V, C, Y, Q R o o$ \\
\hline Mateo-Cid et al., 2013a & $\mathrm{C}$ \\
\hline Mateo-Cid et al., 2013b & $\mathrm{C}$ \\
\hline Mateo-Cid et al., 2014b & V, Y, QRoo \\
\hline Mateo-Cid et al., 2016 & C, QRoo \\
\hline
\end{tabular}


Apéndice 1. Continuación.

\begin{tabular}{|c|c|}
\hline Publicaciones & Estados \\
\hline Mateo Cid et al., 2018 & $\mathrm{~V}$ \\
\hline Mendoza-González y Mateo-Cid, 1992 & QRoo \\
\hline Mendoza-González et al., 2016 & $C, Y, Q R o o$ \\
\hline Mendoza-González et al., 2007 & QRoo \\
\hline Mendoza-González et al., 2013 & C \\
\hline Mendoza-González et al., 2017 & $\mathrm{~Tb}$ \\
\hline Núñez-Resendiz, 2009 & V \\
\hline Núñez-Resendiz et al., 2015 & C, Y, QRoo \\
\hline Núñez-Resendiz et al., 2017a & $\mathrm{T}, \mathrm{V}, \mathrm{Tb}, \mathrm{C}, \mathrm{Y}, \mathrm{QRoo}$ \\
\hline Núñez-Resendiz et al., 2017b & C, QRoo \\
\hline Núñez-Resendiz et al., 2019a & C, Y, QRoo \\
\hline Núñez-Resendiz et al., 2019b & C, Y, QRoo \\
\hline Núñez-Resendiz et al., 2019c & C, QRoo \\
\hline Núñez-Resendiz et al., 2020 & C \\
\hline Ortega, 1995 & C \\
\hline Ortegón-Aznar et al., 2001 & $\mathrm{Y}$ \\
\hline Ortegón-Aznar et al., 2009 & $\mathrm{Y}$ \\
\hline Palma-Ortiz et al., 2017 & C \\
\hline Quan-Young et al., 2006 & QRoo \\
\hline Quiroz-González et al., 2018 & $\mathrm{~Tb}$ \\
\hline Ramírez-López, 1996 & $\mathrm{~Tb}$ \\
\hline Sánchez-Rodríguez, 1980 & V \\
\hline Sentíes et al., 2001 & QRoo \\
\hline Sentíes y Díaz-Larrea, 2008 & QRoo \\
\hline Sentíes et al., 2009 & QRoo \\
\hline Sentíes et al., 2011 & QRoo \\
\hline Sentíes y Dreckmann, 2013 & $\mathrm{~Tb}$ \\
\hline Sentíes et al., 2015 & QRoo \\
\hline Sentíes et al., 2016 & QRoo \\
\hline Vargas López et al., 2004 & $T$ \\
\hline Vilchis et al., 2018 & $\mathrm{~T}, \mathrm{~V}, \mathrm{~Tb}, \mathrm{C}, \mathrm{Y}, \mathrm{QRoo}$ \\
\hline Zizumbo Alamilla, 1995 & V \\
\hline
\end{tabular}


Apéndice 2. Especies en negritas representan registros nuevos posteriores al catálogo de Ortega et al., 2001. Las especies en color azul representan publicaciones previas a 2001, no contenidas en el mencionado catálogo y que no vuelven a ser reportadas posteriores a la fecha de su registro. Abreviaciones: Tamaulipas (T), Veracruz (V), Tabasco (Tb), Campeche (C), Yucatán (Y) y Quintana Roo (QRoo). Región marítima G=Golfo de México, $\mathrm{C}=$ Caribe y $\mathrm{A}=\operatorname{ambos}(\mathrm{G}$ y $\mathrm{C})$.

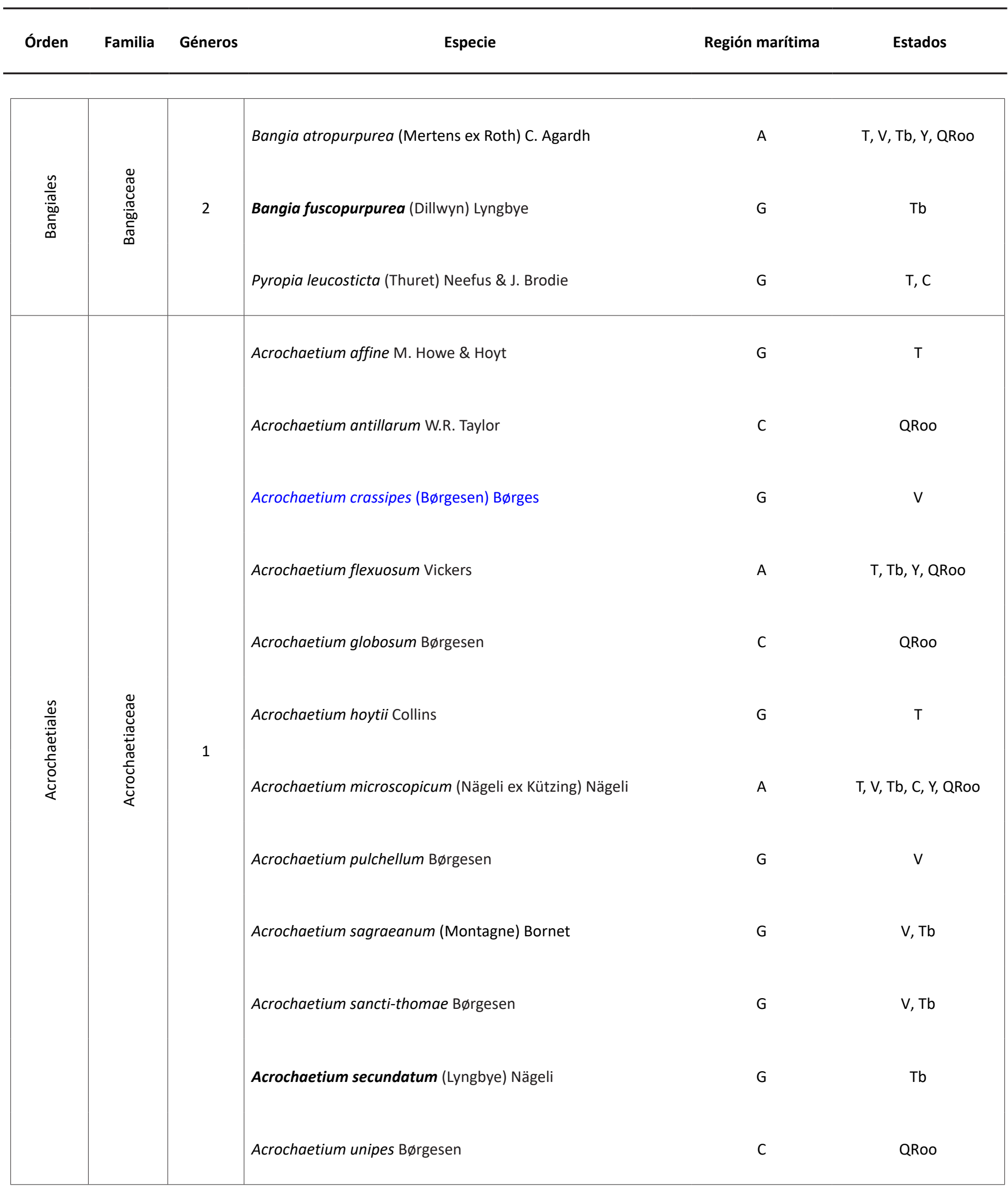


Apéndice 2. Continuación.

Órden Familia Géneros Especie Región marítima

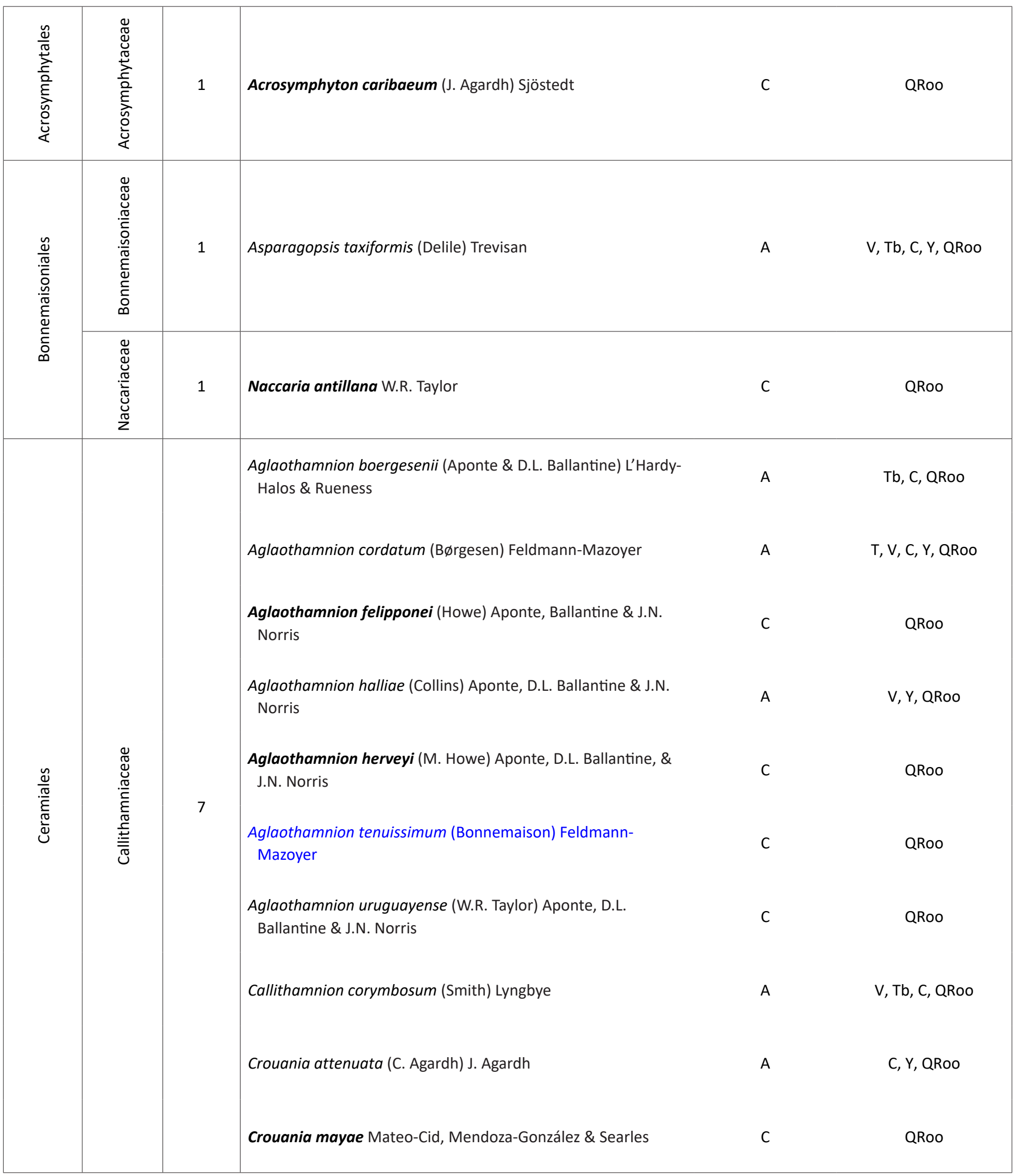


Apéndice 2. Continuación.

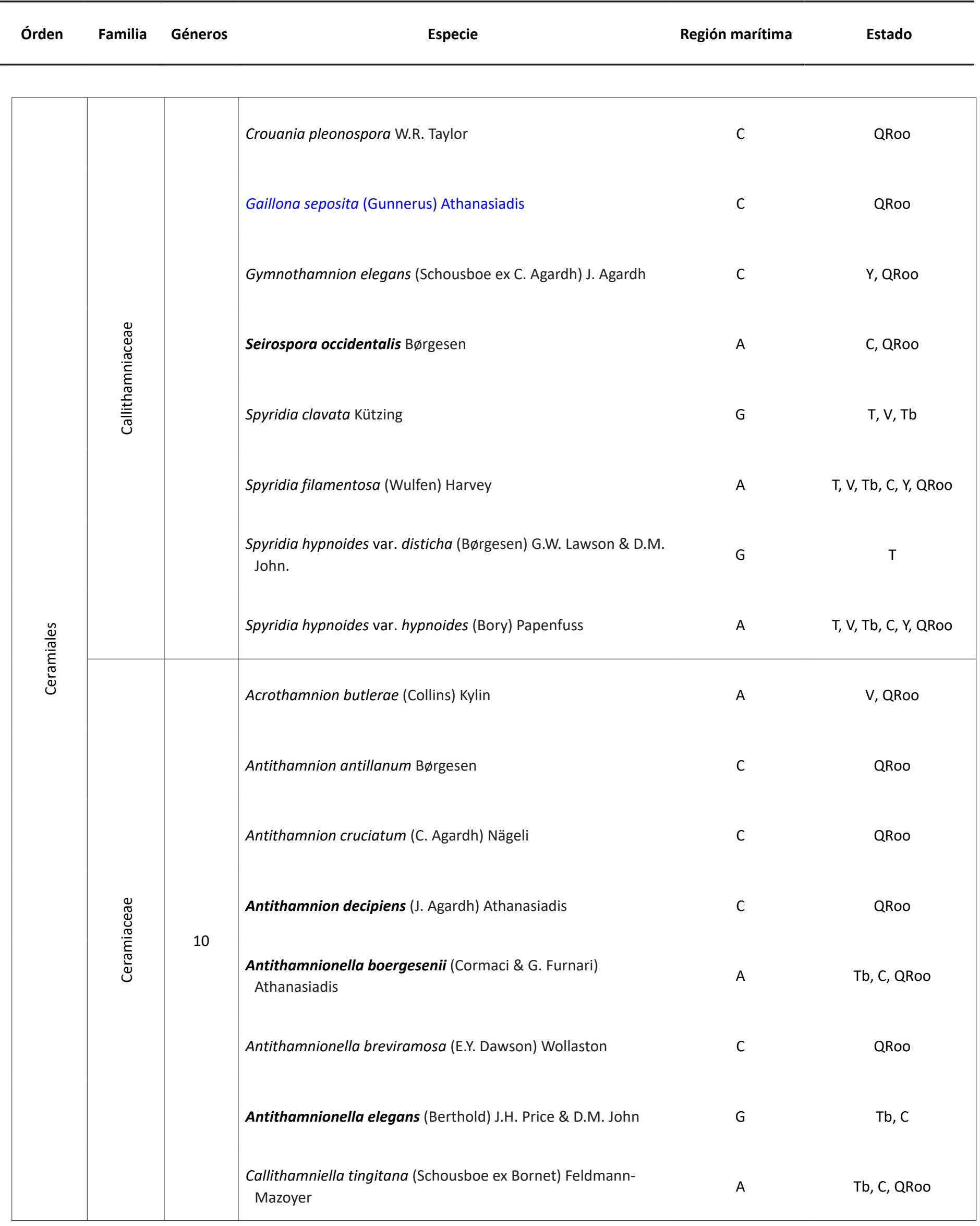


Apéndice 2. Continuación.

\begin{tabular}{|c|c|c|c|c|c|}
\hline Órden & Familia & Géneros & Especie & Región marítima & Estado \\
\hline
\end{tabular}

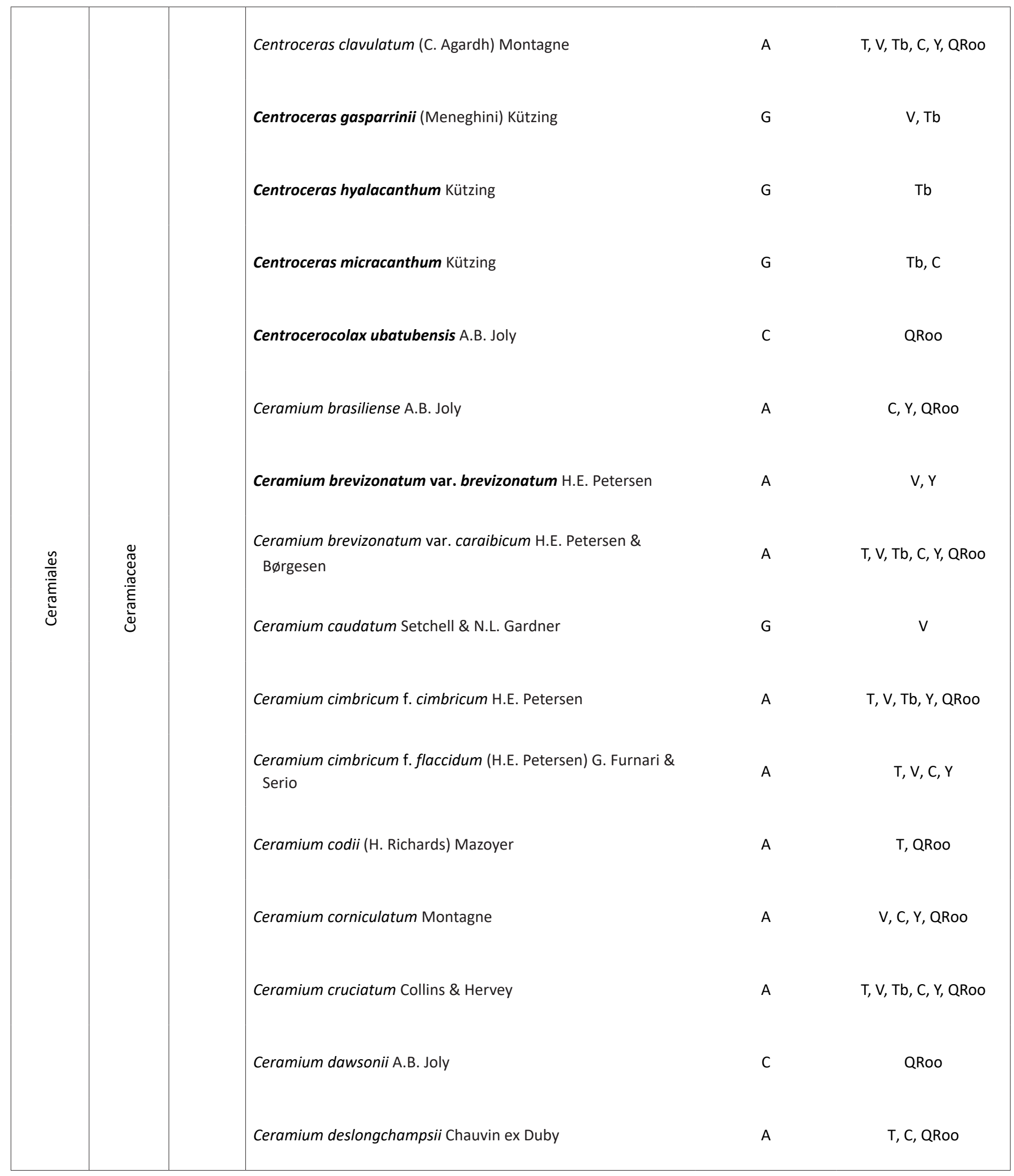


Apéndice 2. Continuación.

\begin{tabular}{|c|c|c|c|c|c|}
\hline Órden & Familia & Géneros & Especie & Región marítima & Estado \\
\hline
\end{tabular}

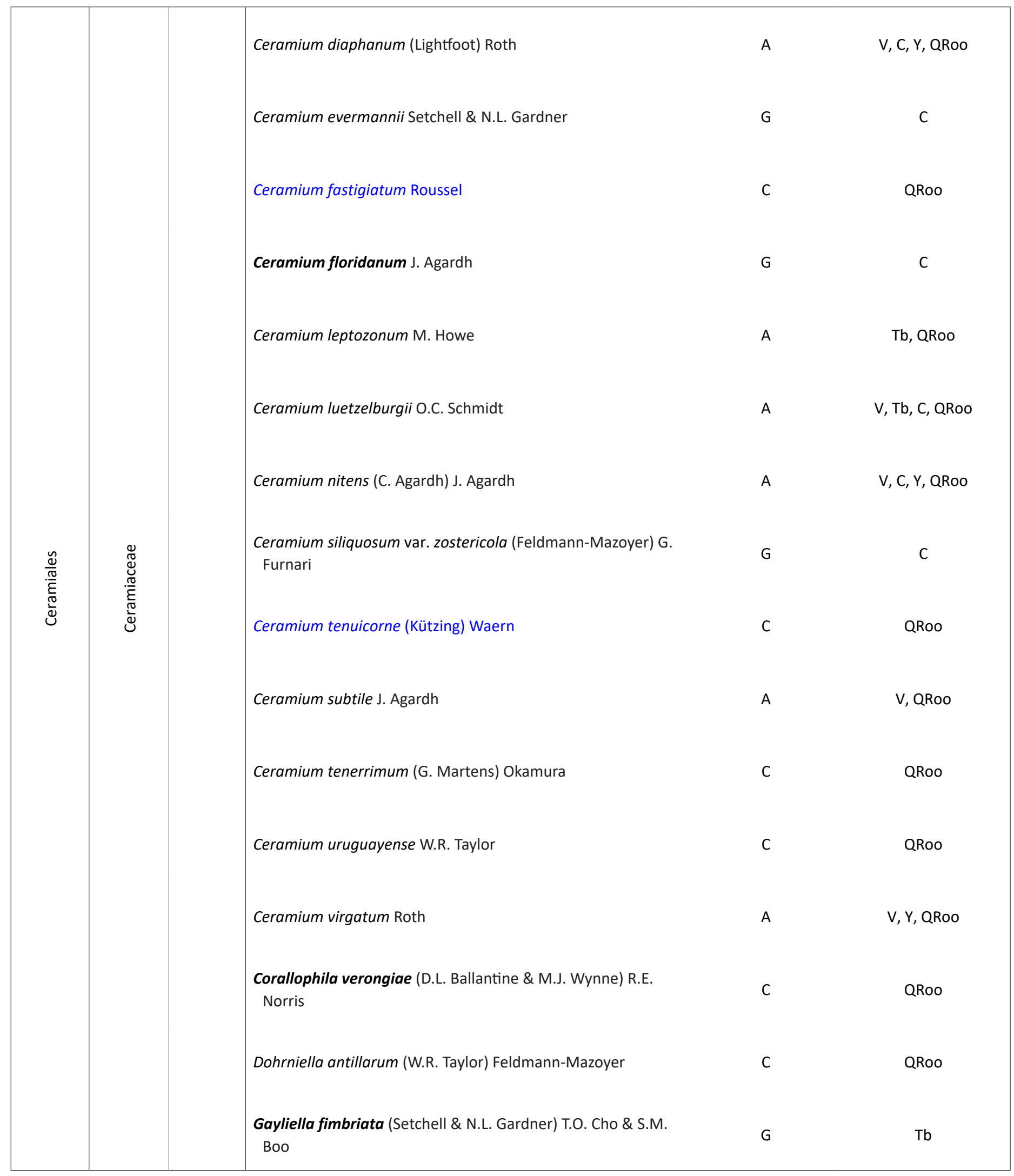


Apéndice 2. Continuación.

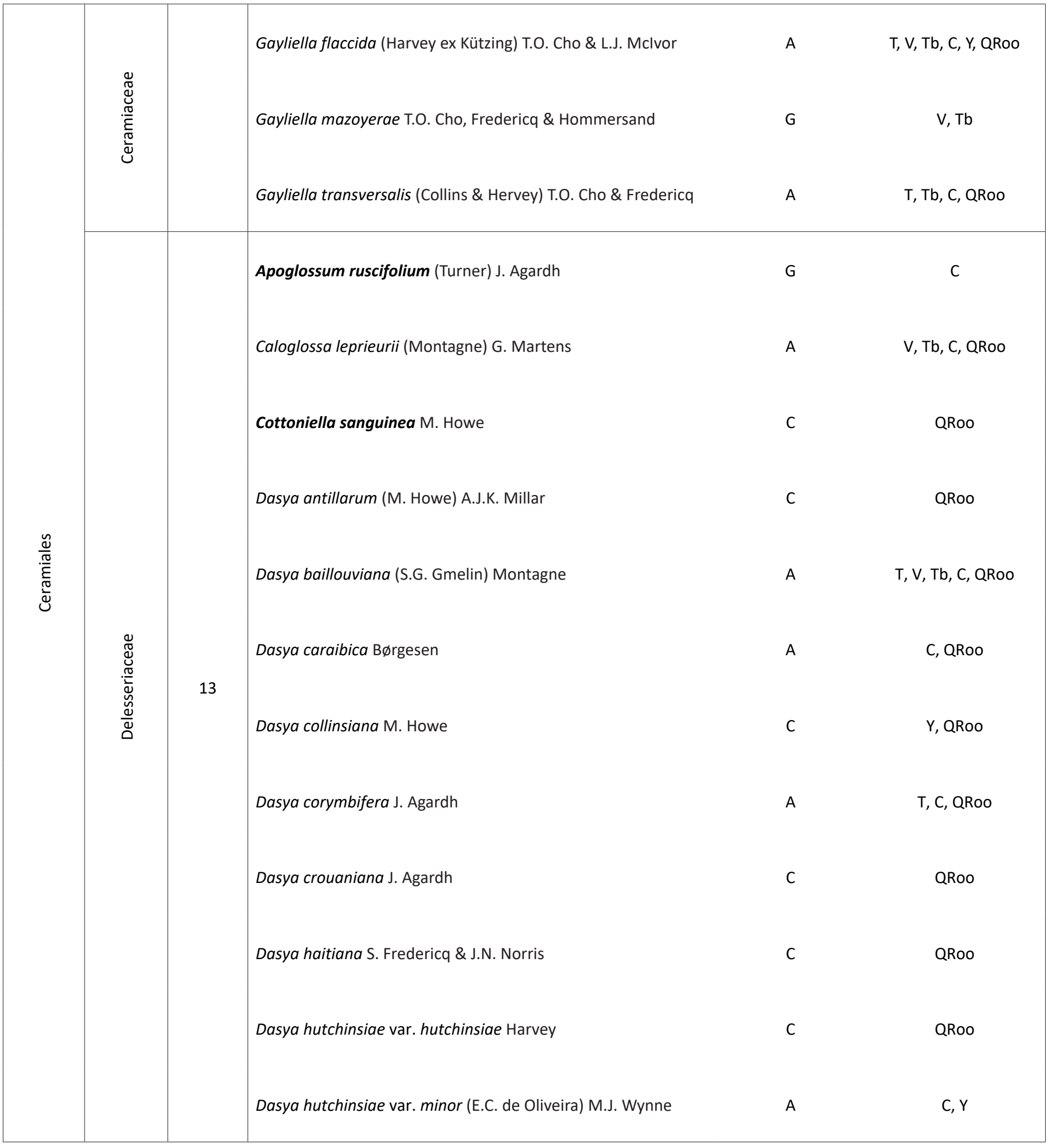


Apéndice 2. Continuación.

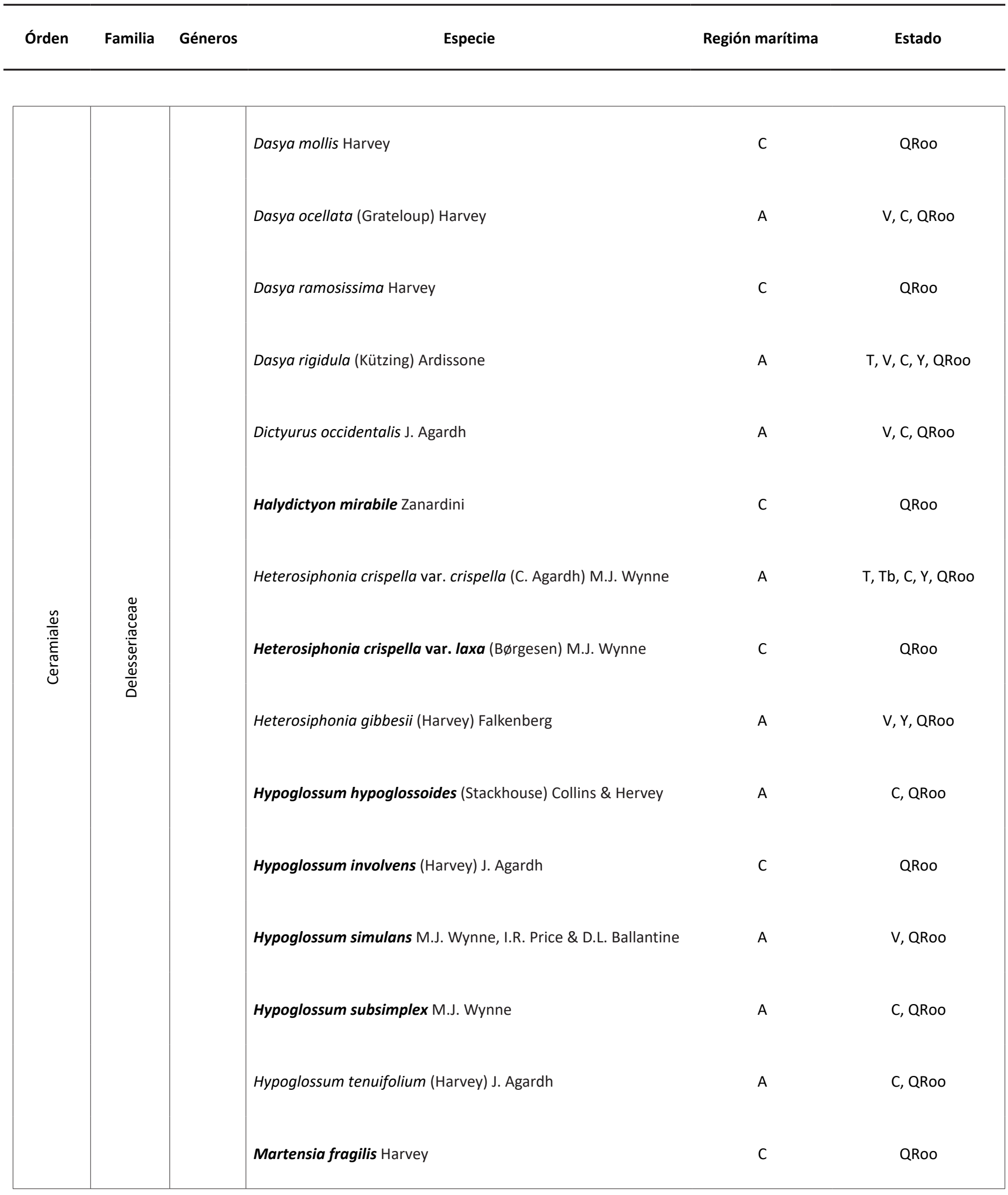


Apéndice 2. Continuación.

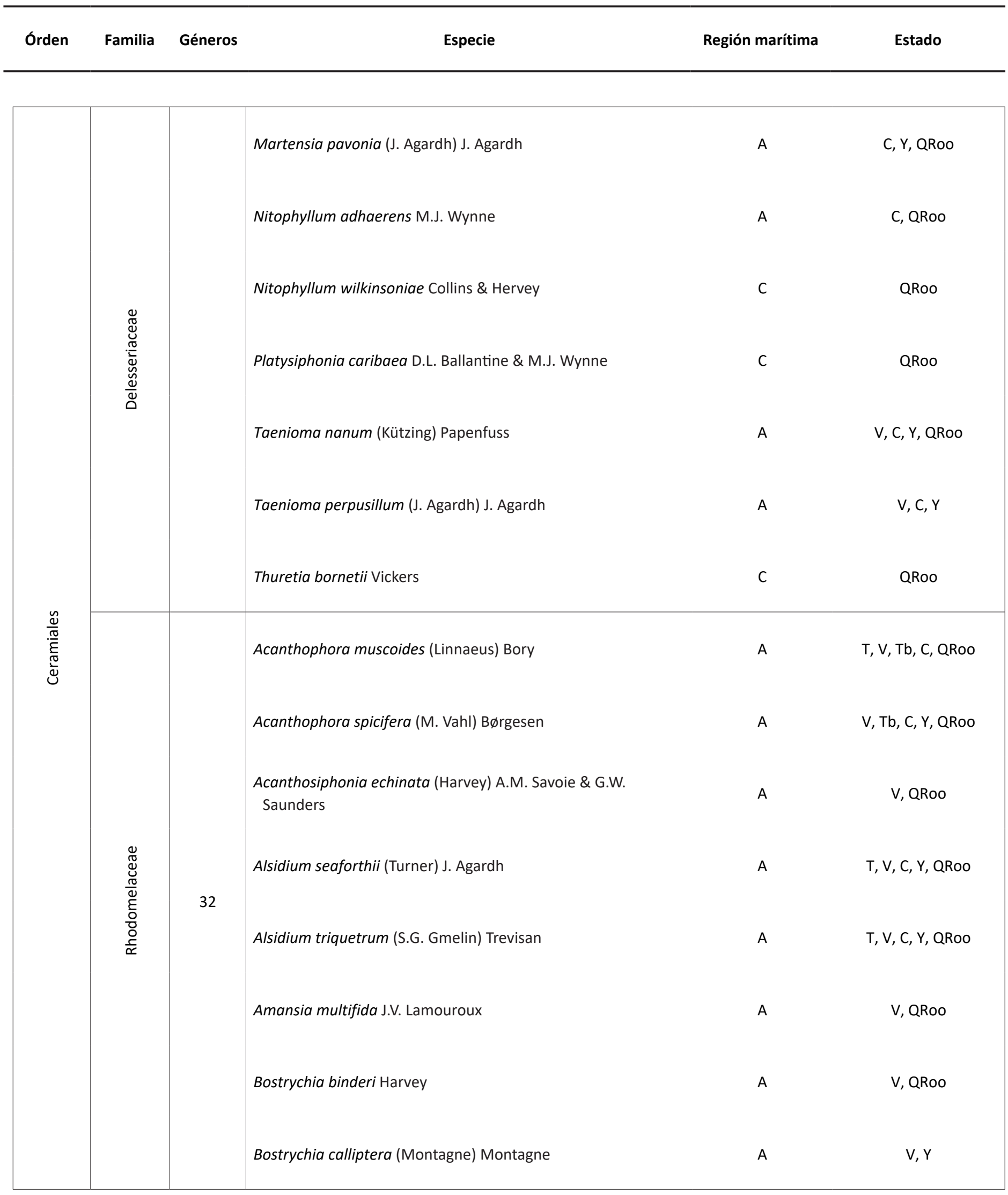


Apéndice 2. Continuación.

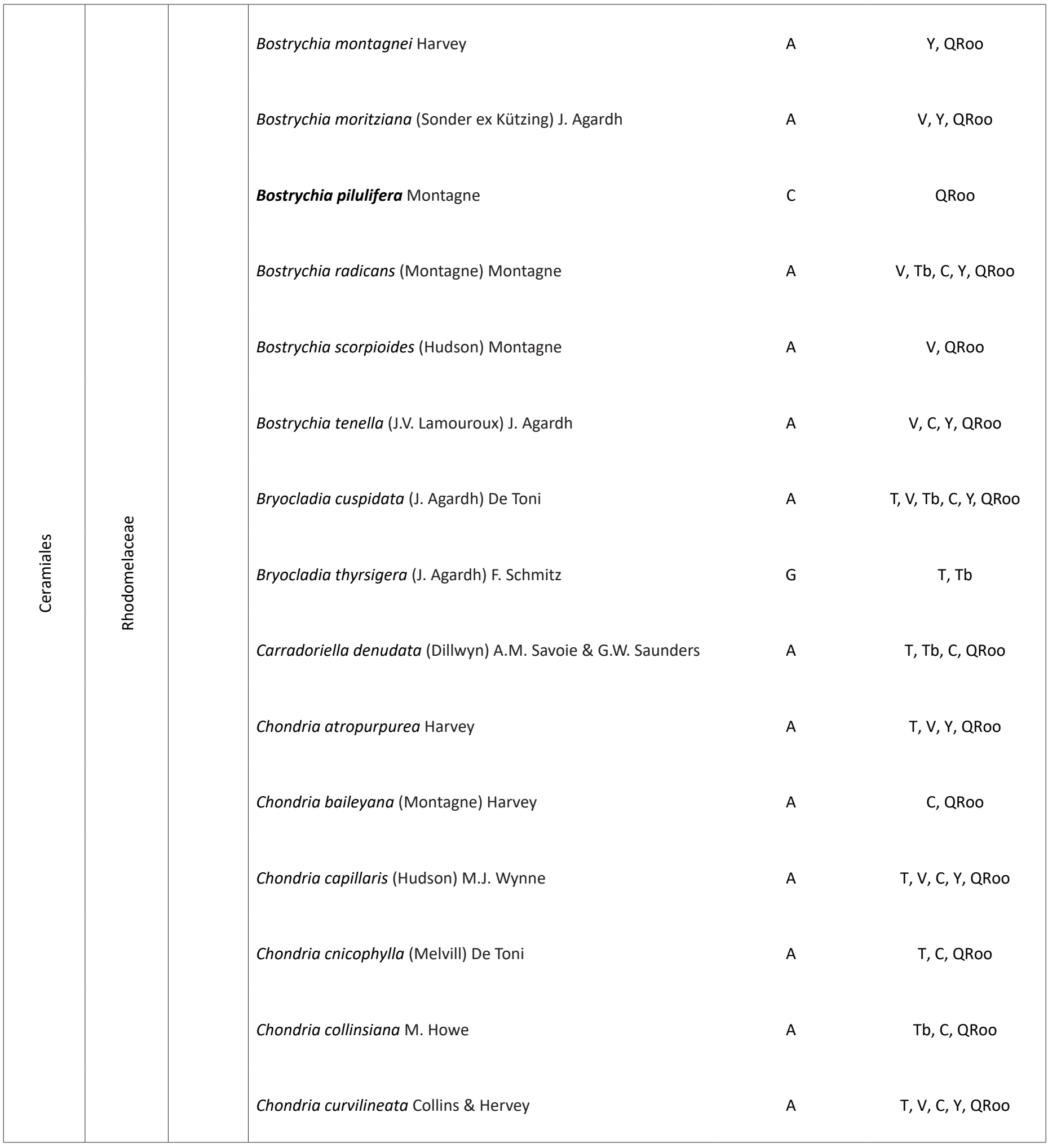


Apéndice 2. Continuación.

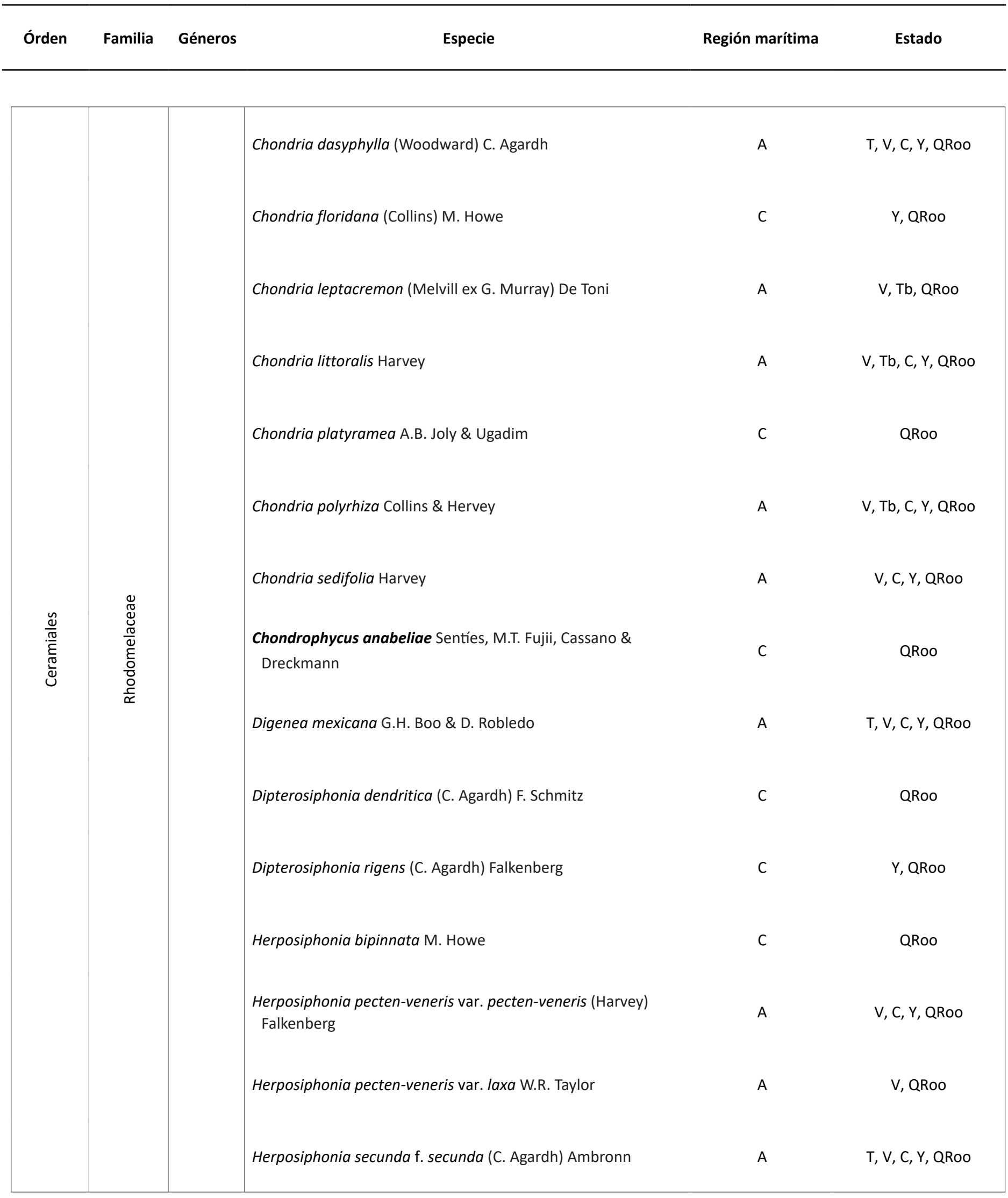


Apéndice 2. Continuación.

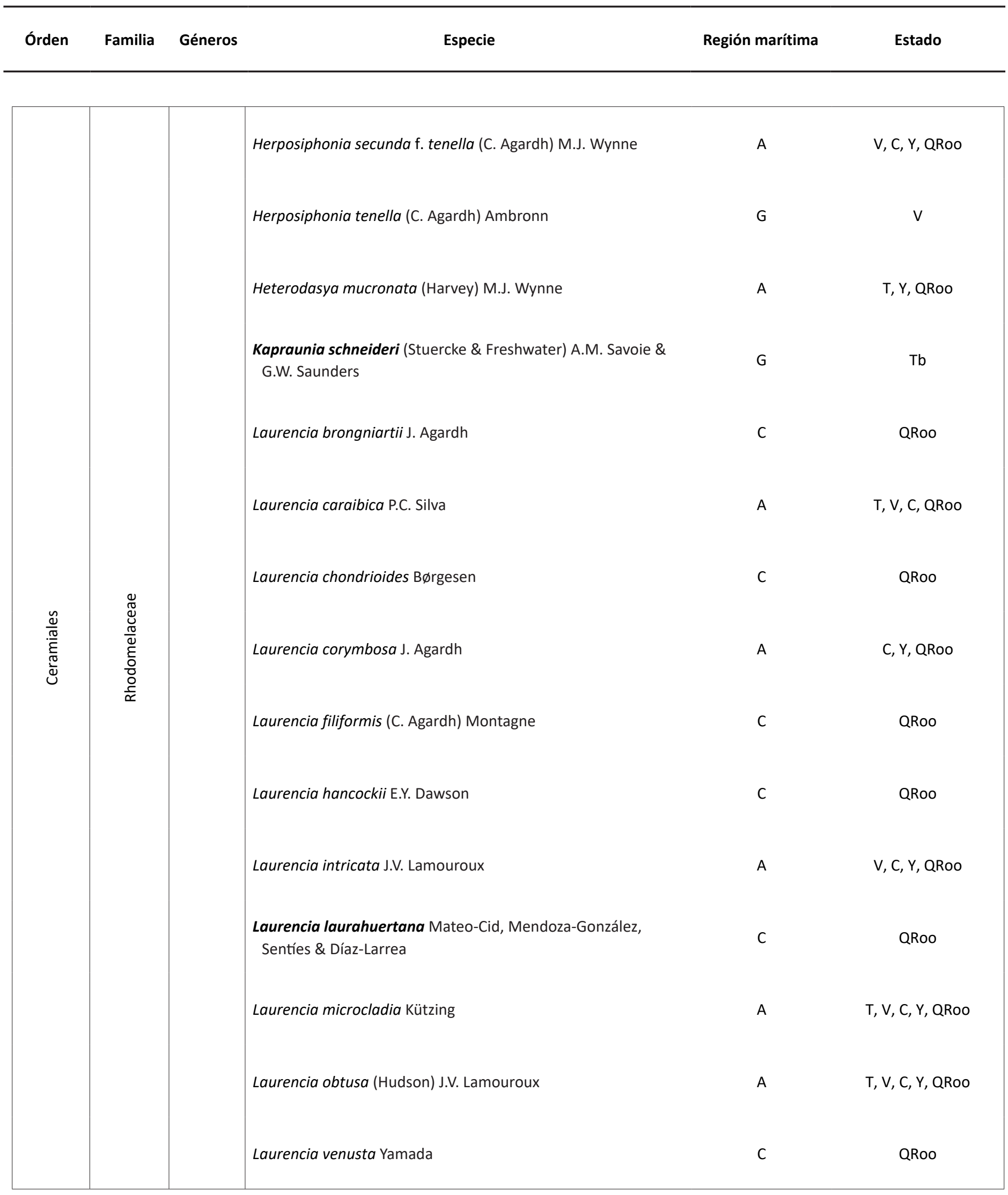


Apéndice 2. Continuación.

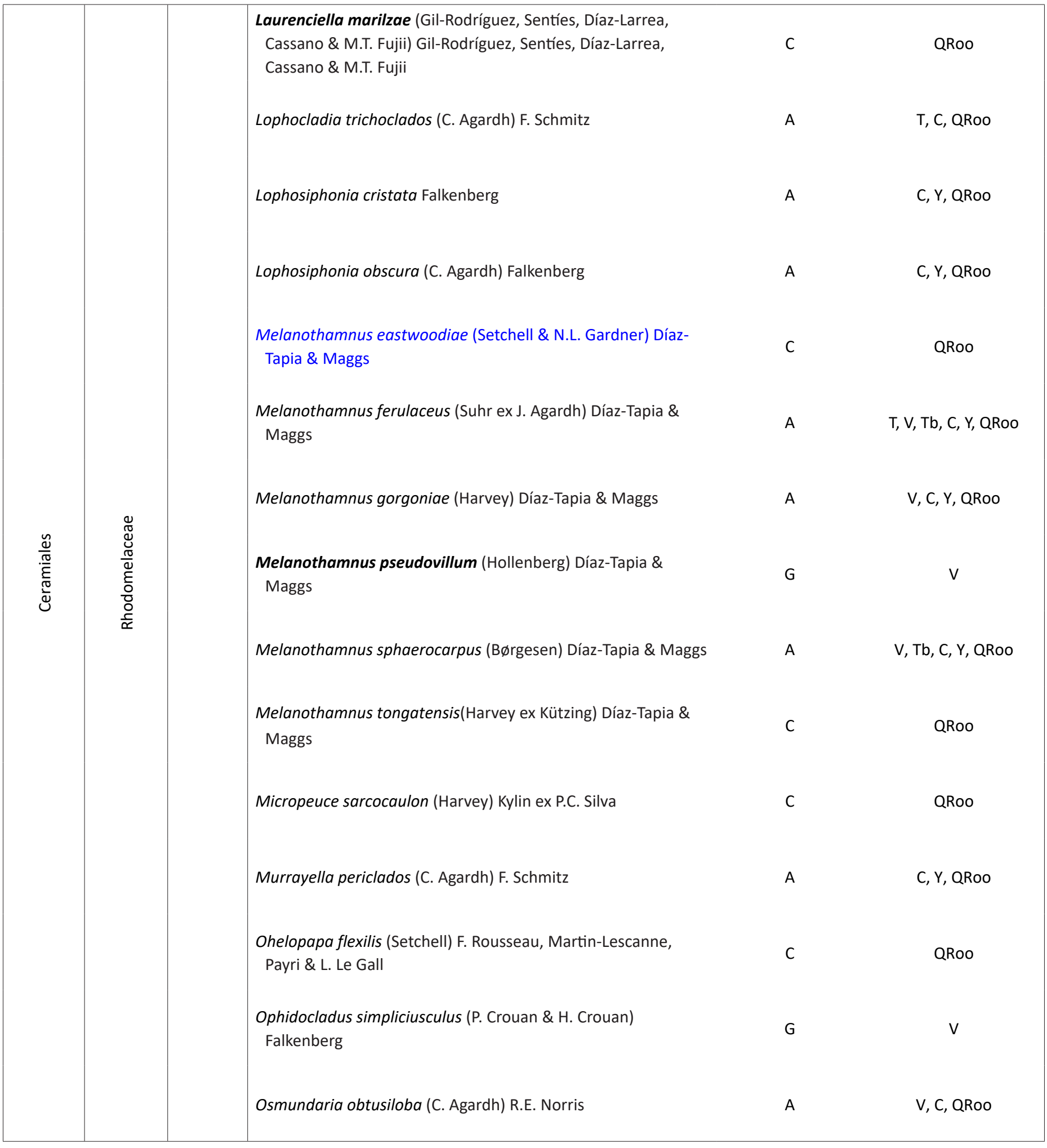


Apéndice 2. Continuación.

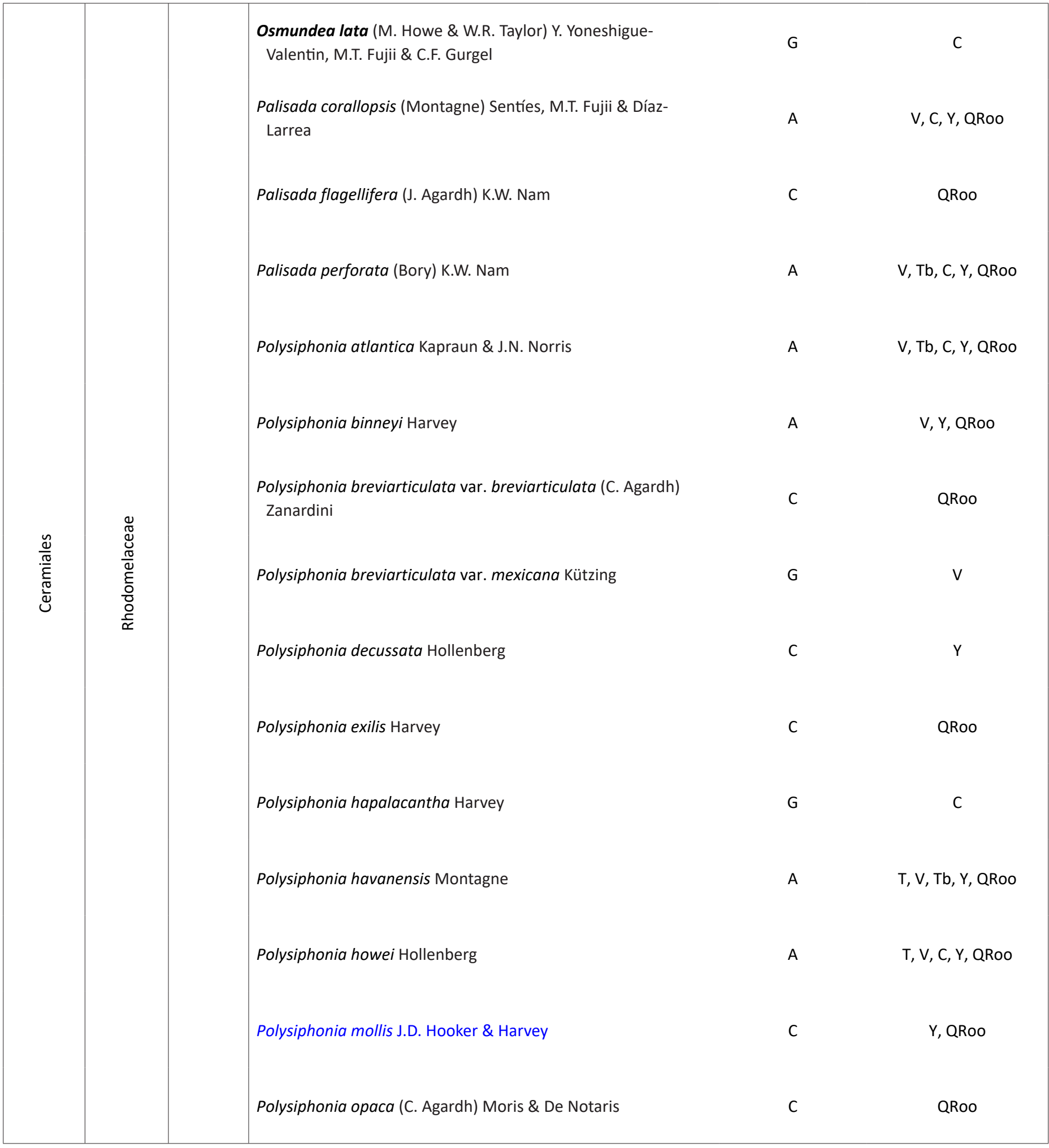


Apéndice 2. Continuación.

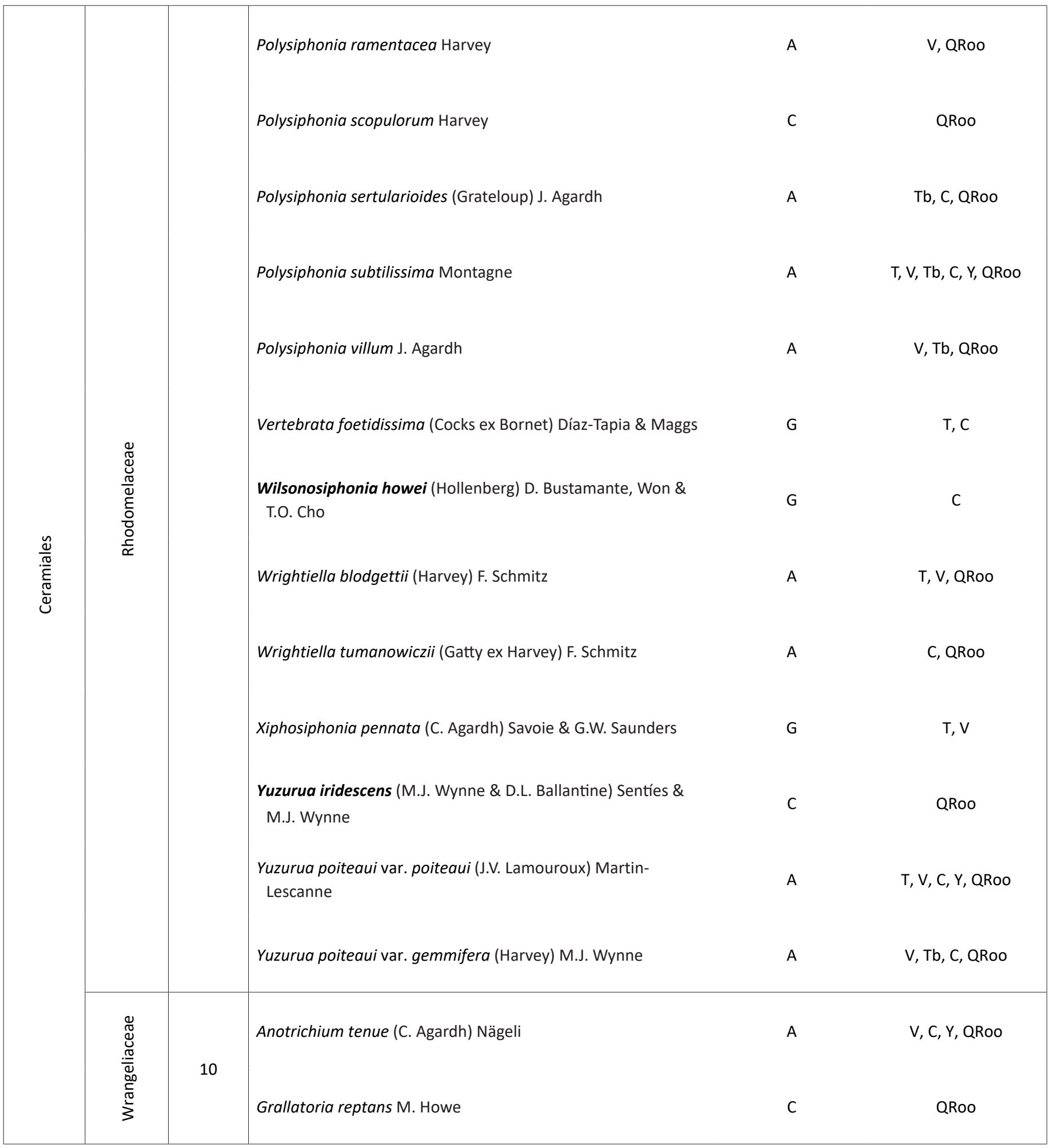


Apéndice 2. Continuación.

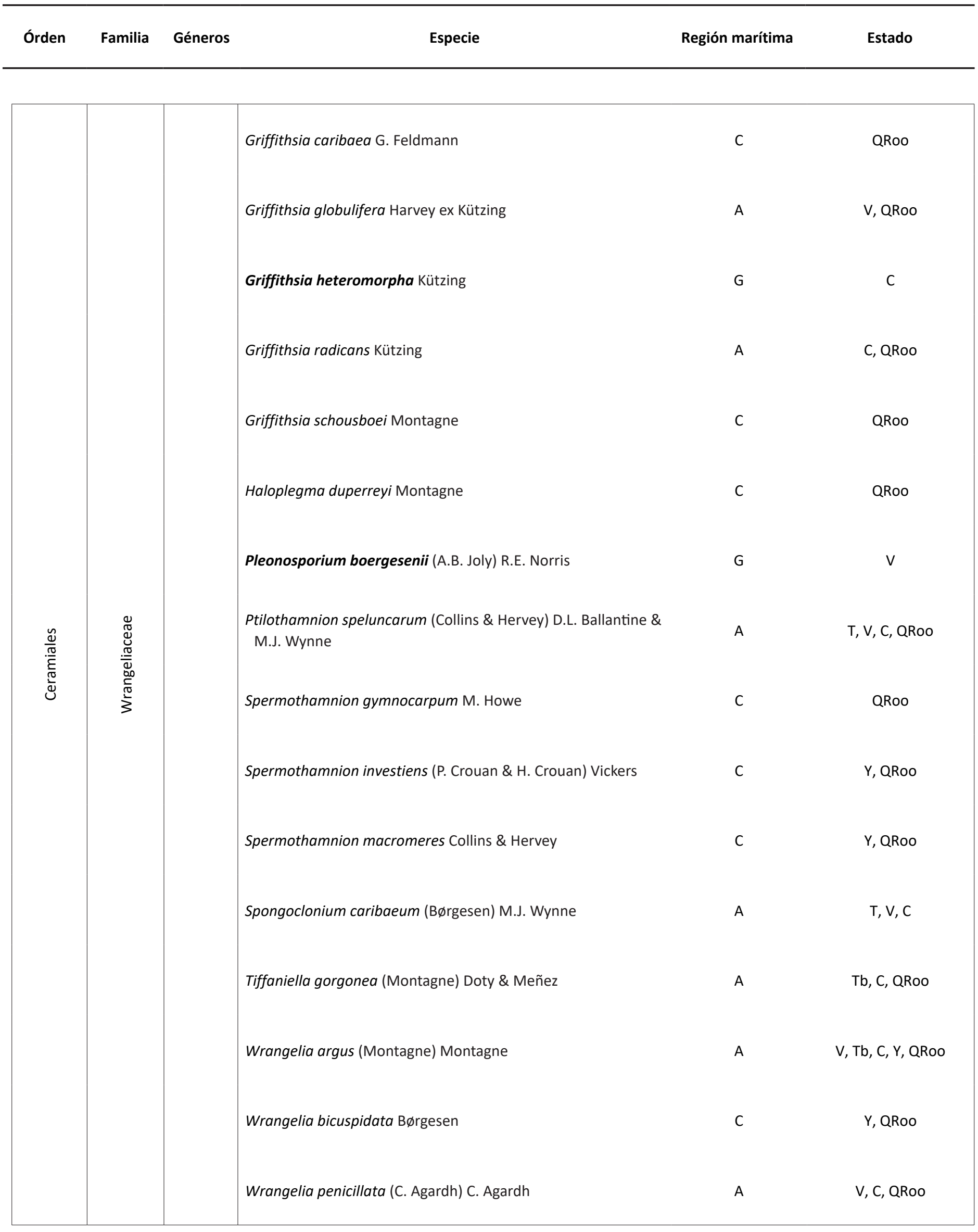


Apéndice 2. Continuación.

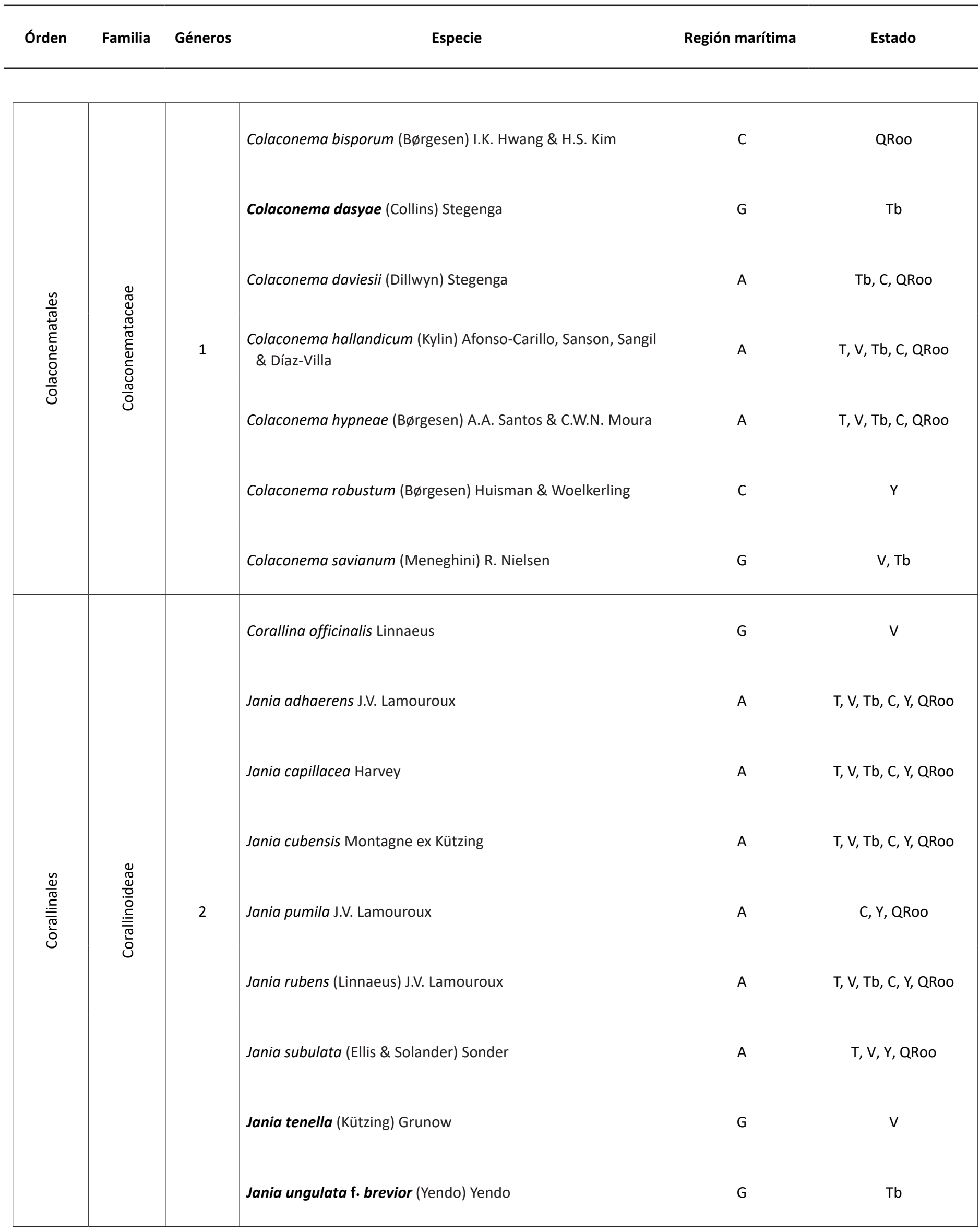


Apéndice 2. Continuación.

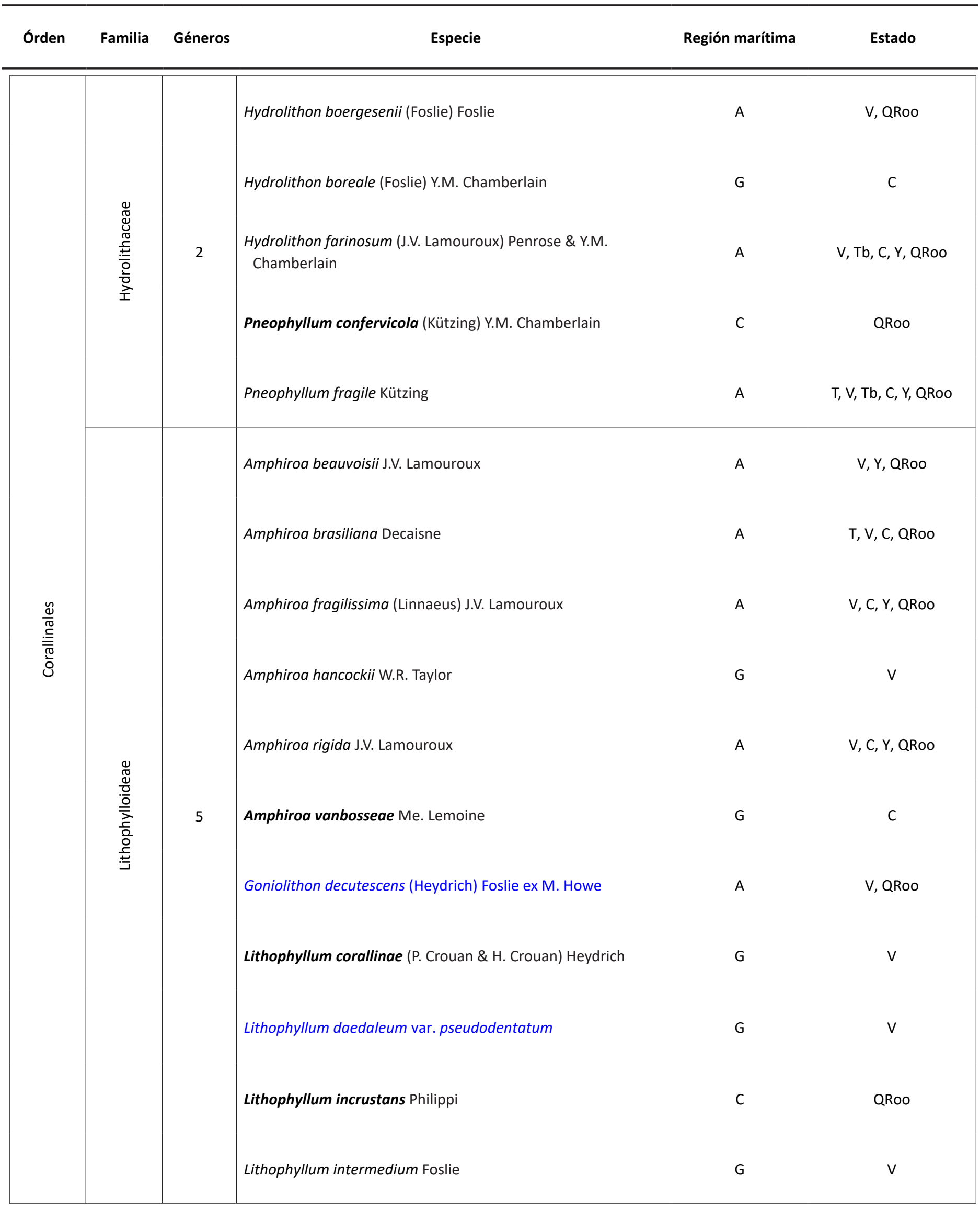


Apéndice 2. Continuación.

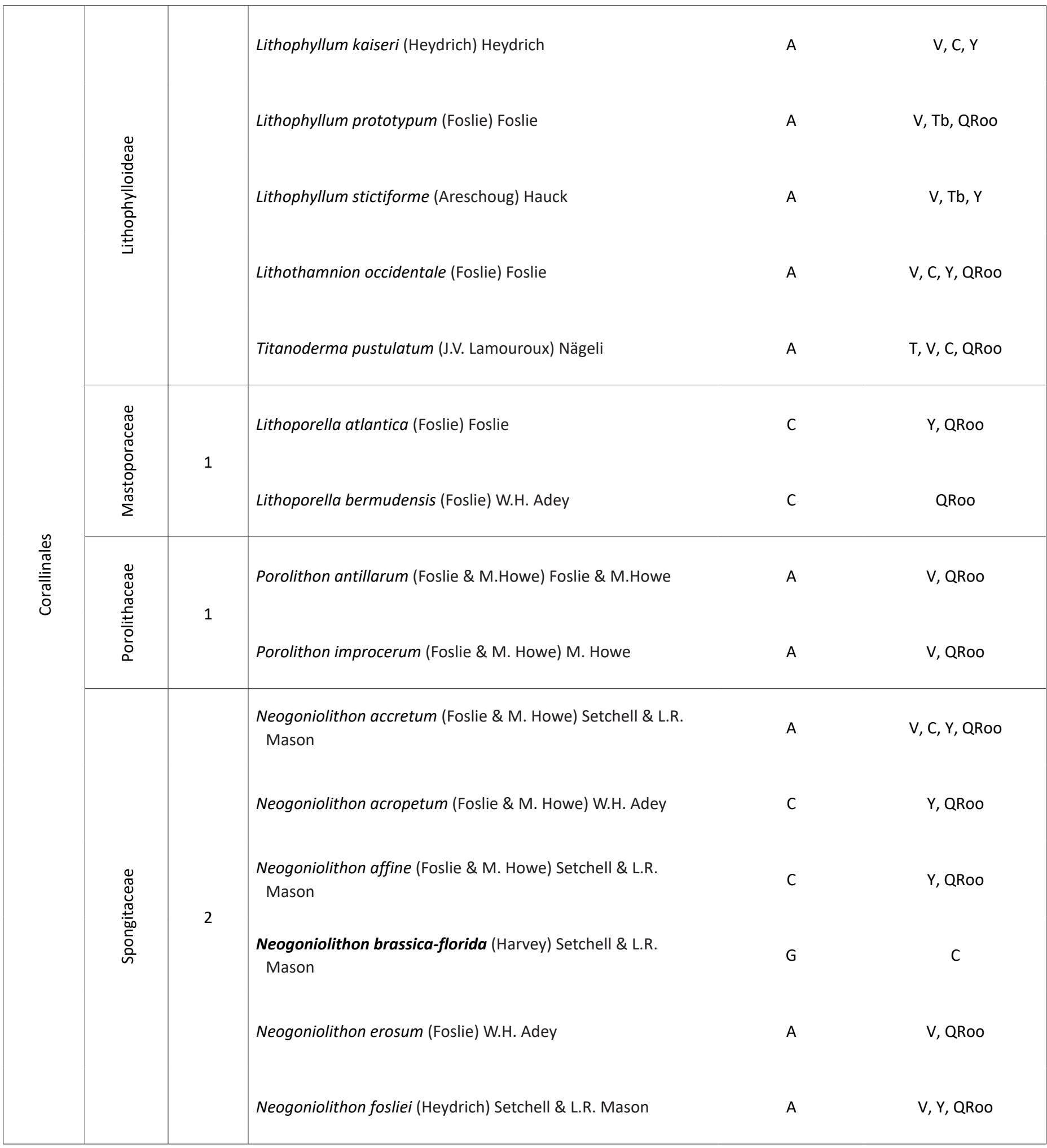


Apéndice 2. Continuación.

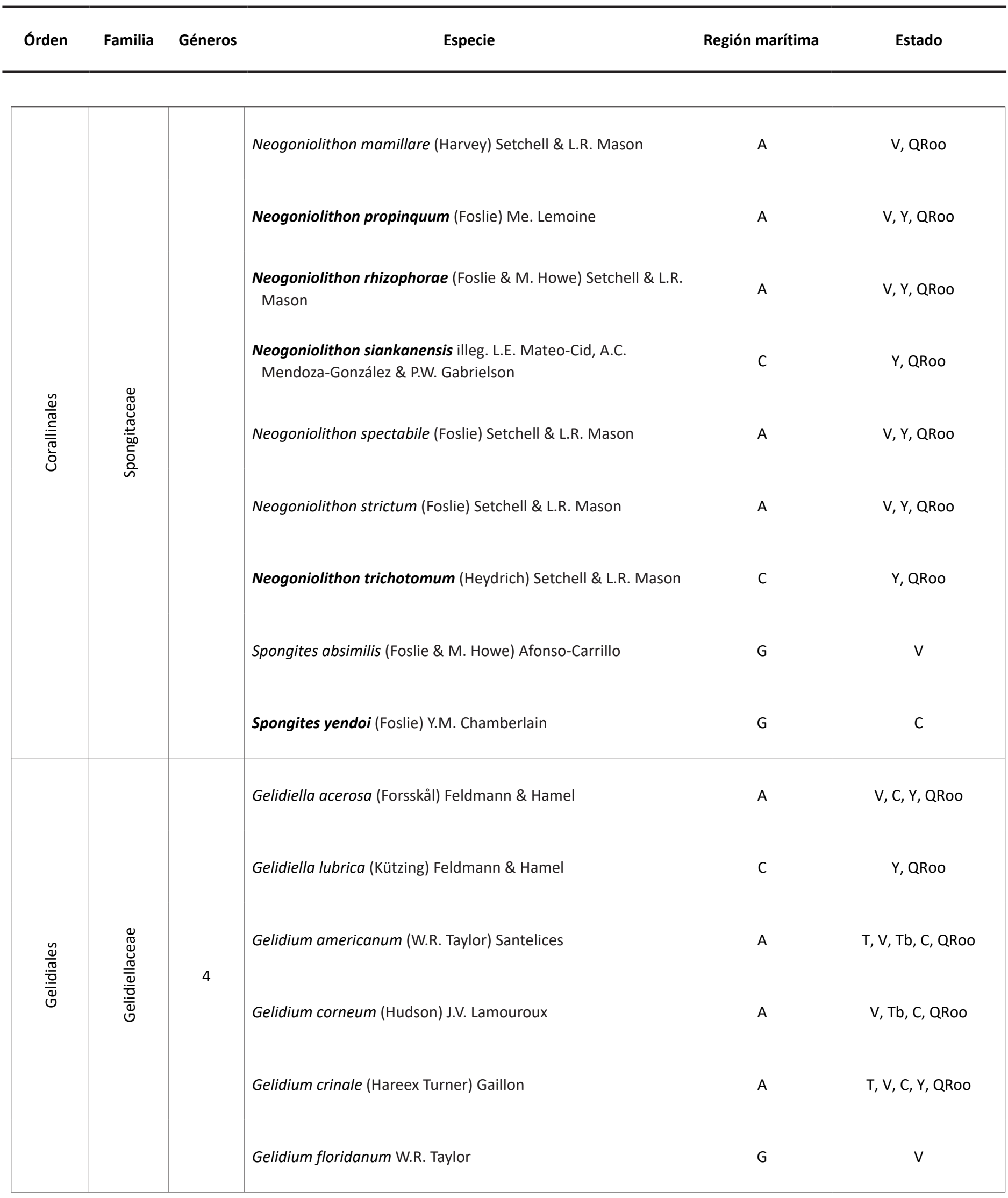


Apéndice 2. Continuación.

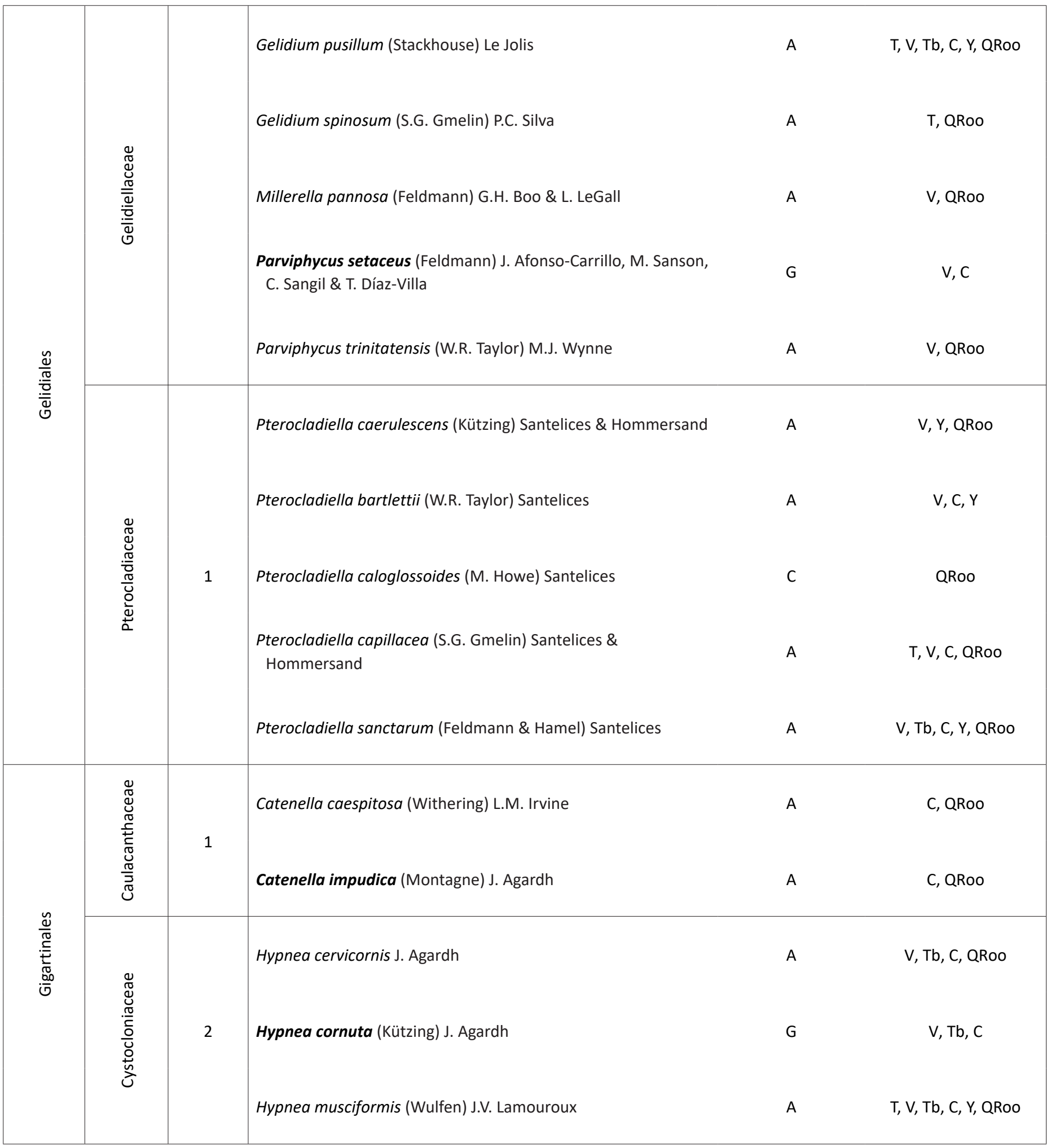


Apéndice 2. Continuación.

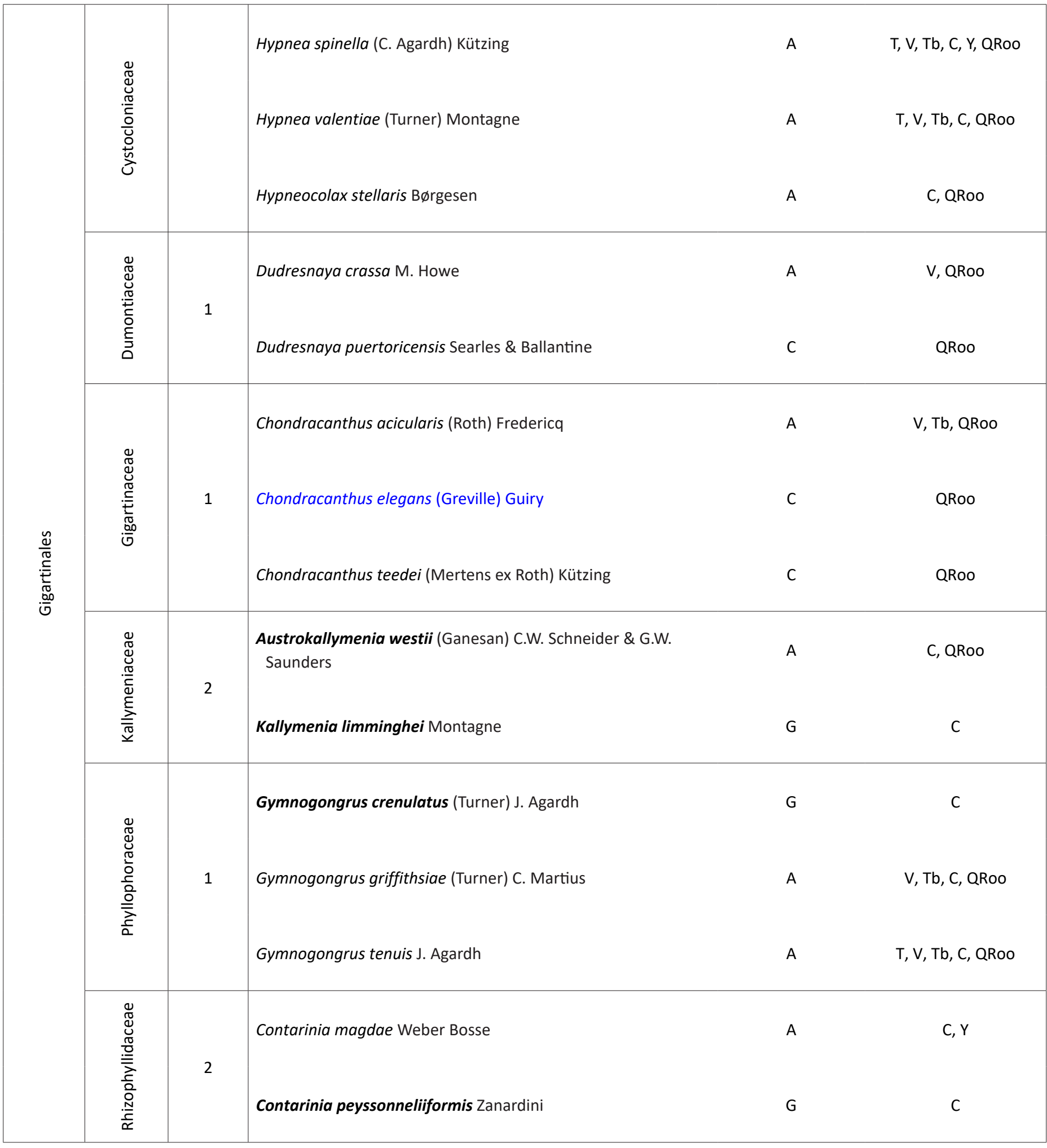


Apéndice 2. Continuación.

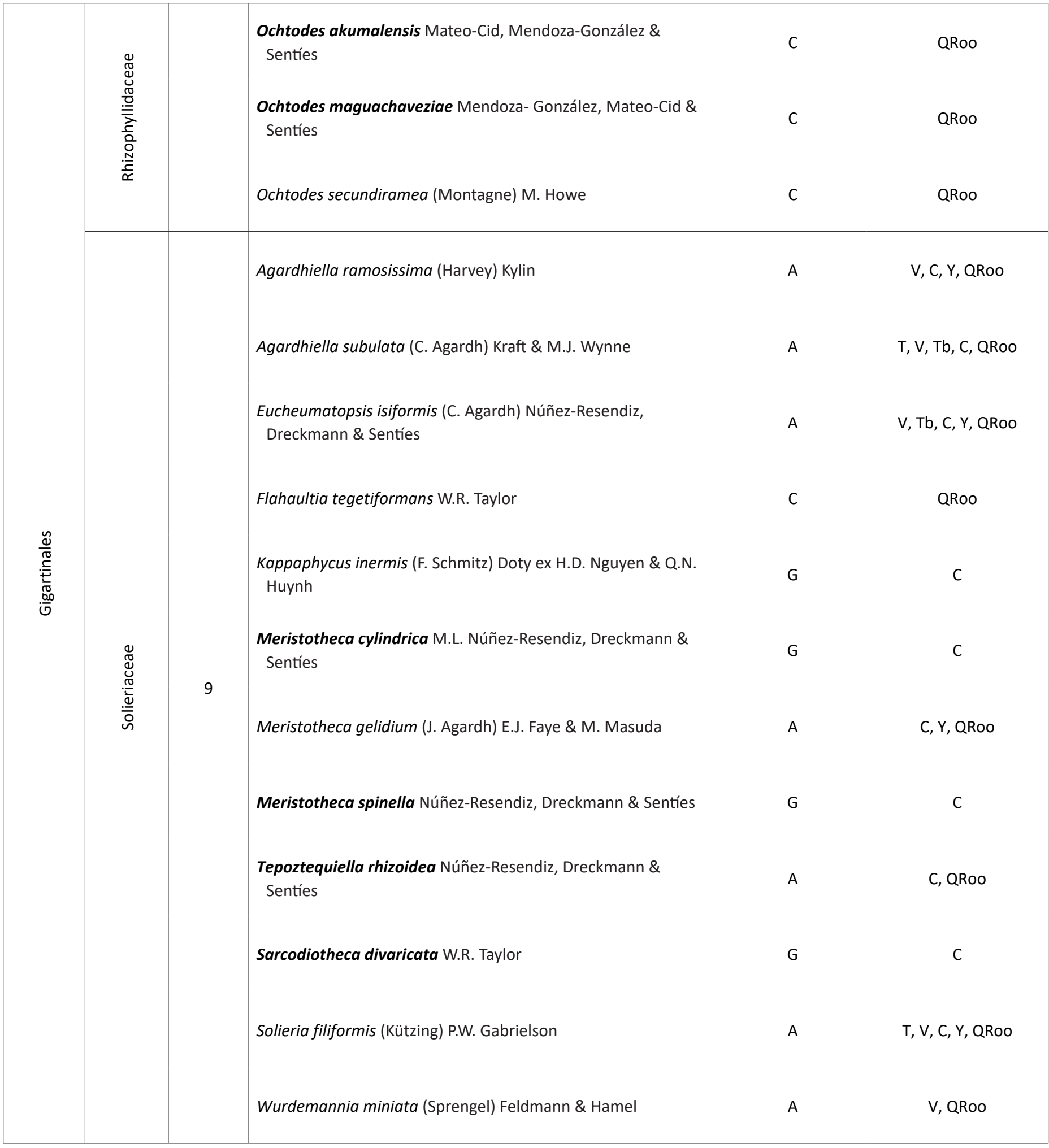


Apéndice 2. Continuación.

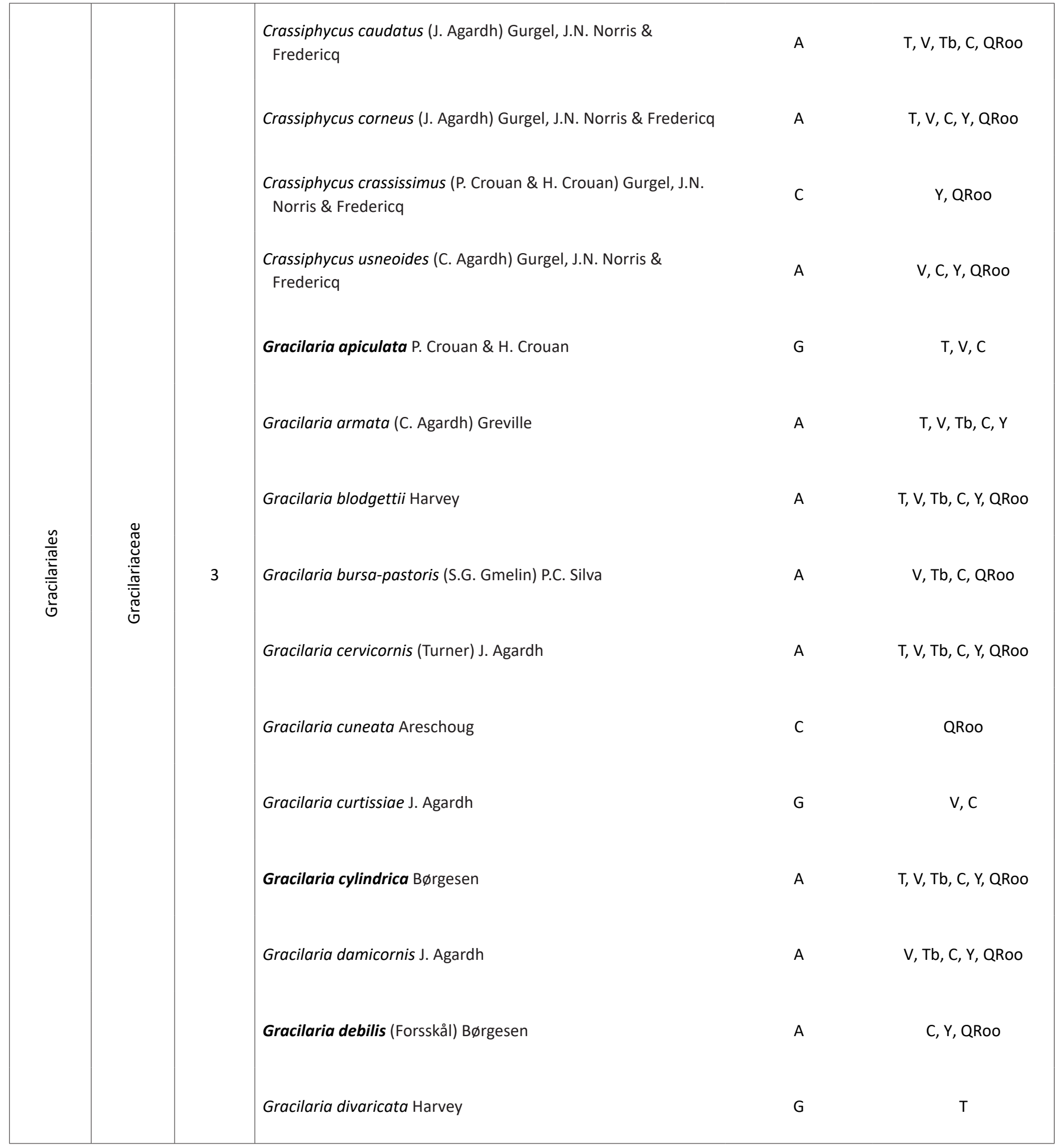


Apéndice 2. Continuación.

\begin{tabular}{|c|c|c|c|c|c|}
\hline Órden & Familia & Géneros & Especie & Región marítima & Estado \\
\hline
\end{tabular}

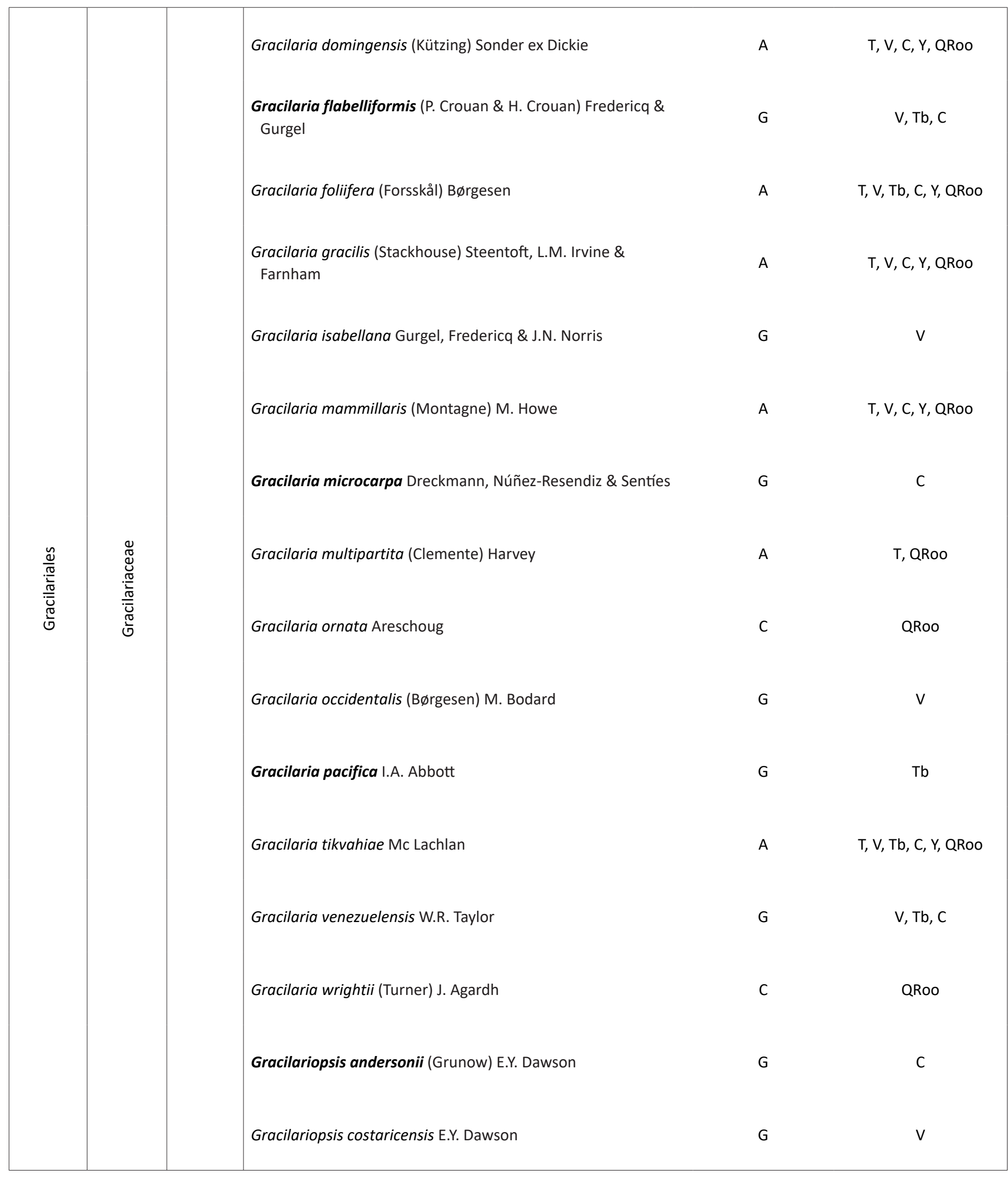


Apéndice 2. Continuación.

Órden Familia Géneros Especie Región marítima

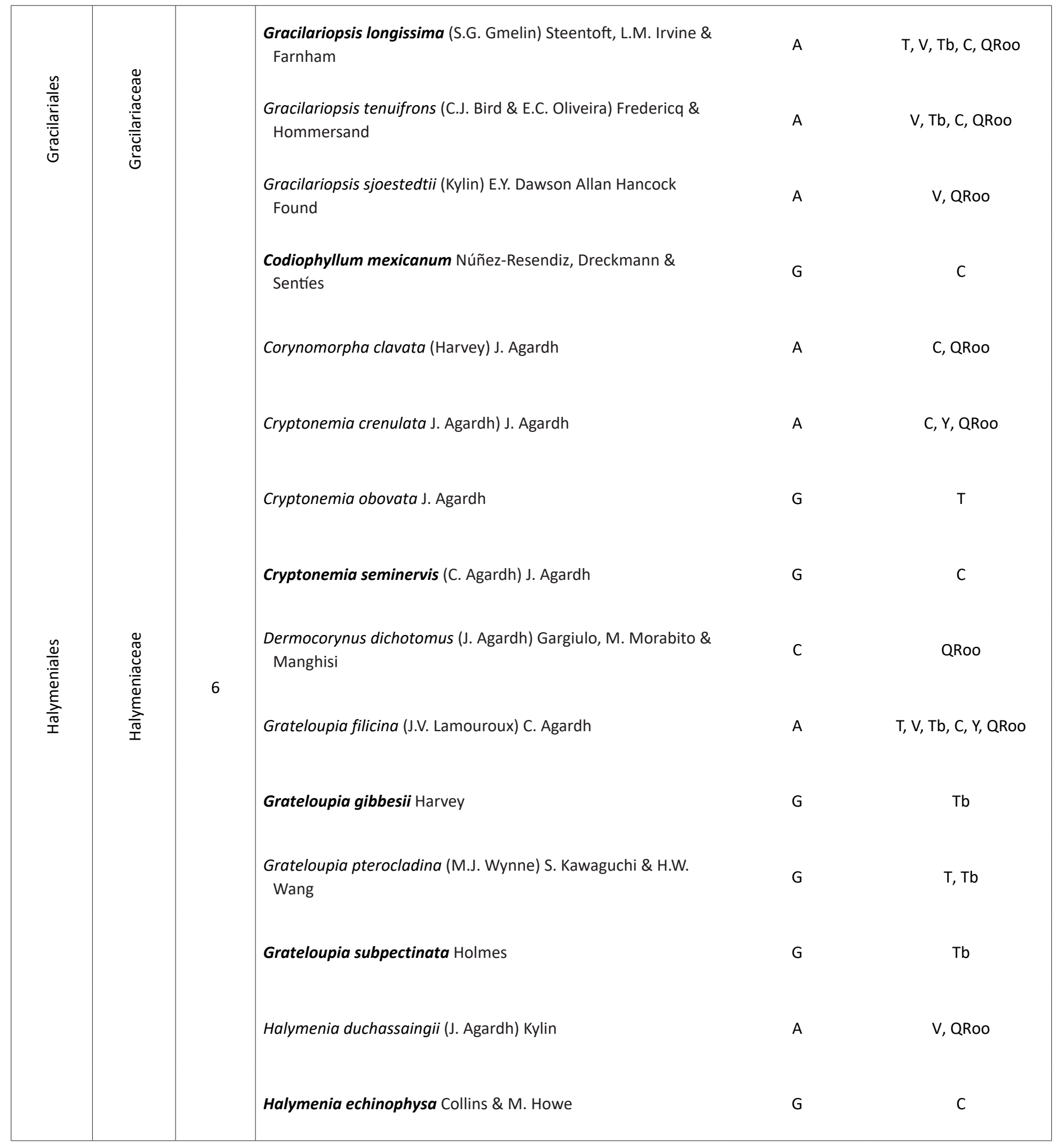


Apéndice 2. Continuación.

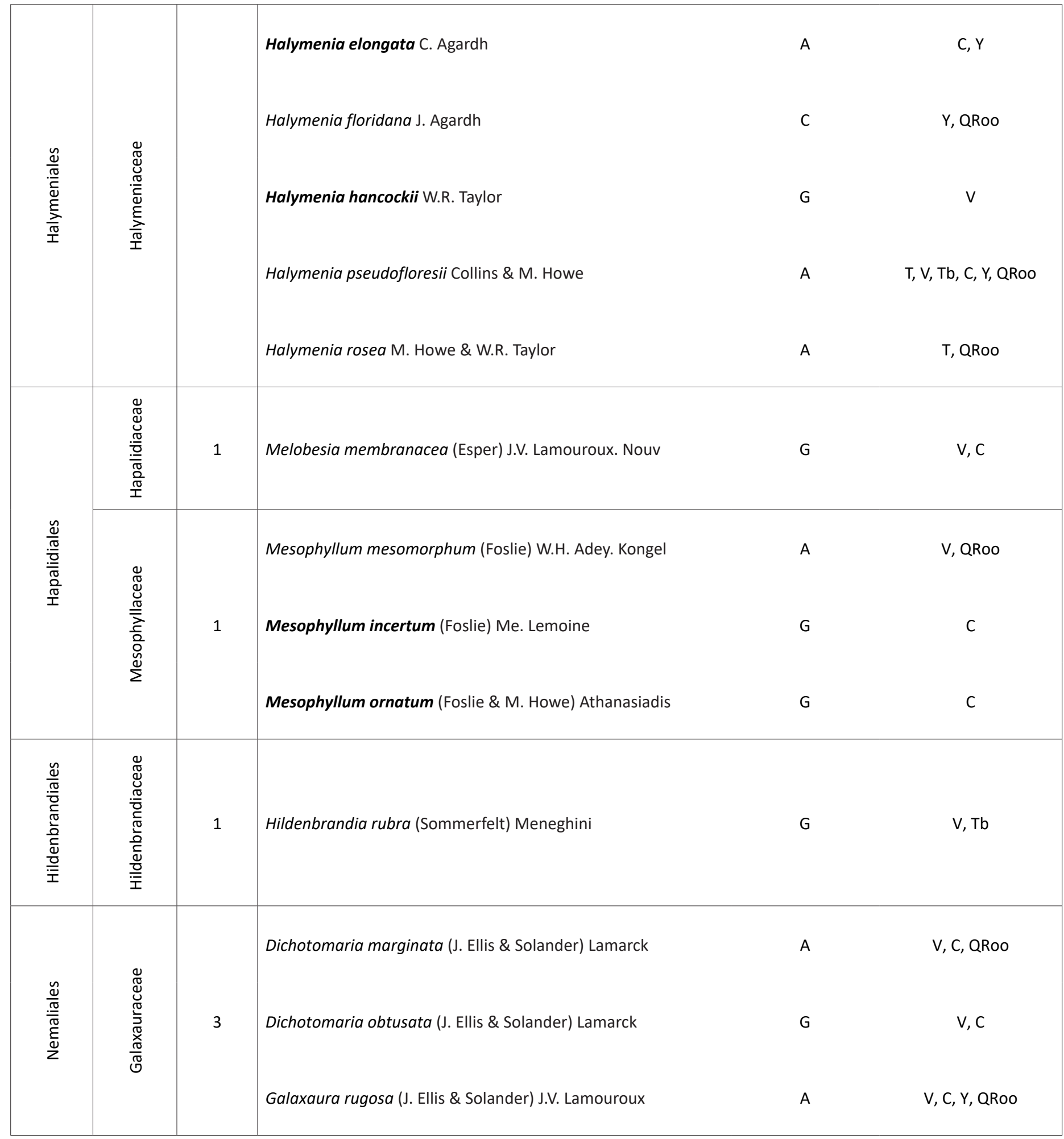


Apéndice 2. Continuación.

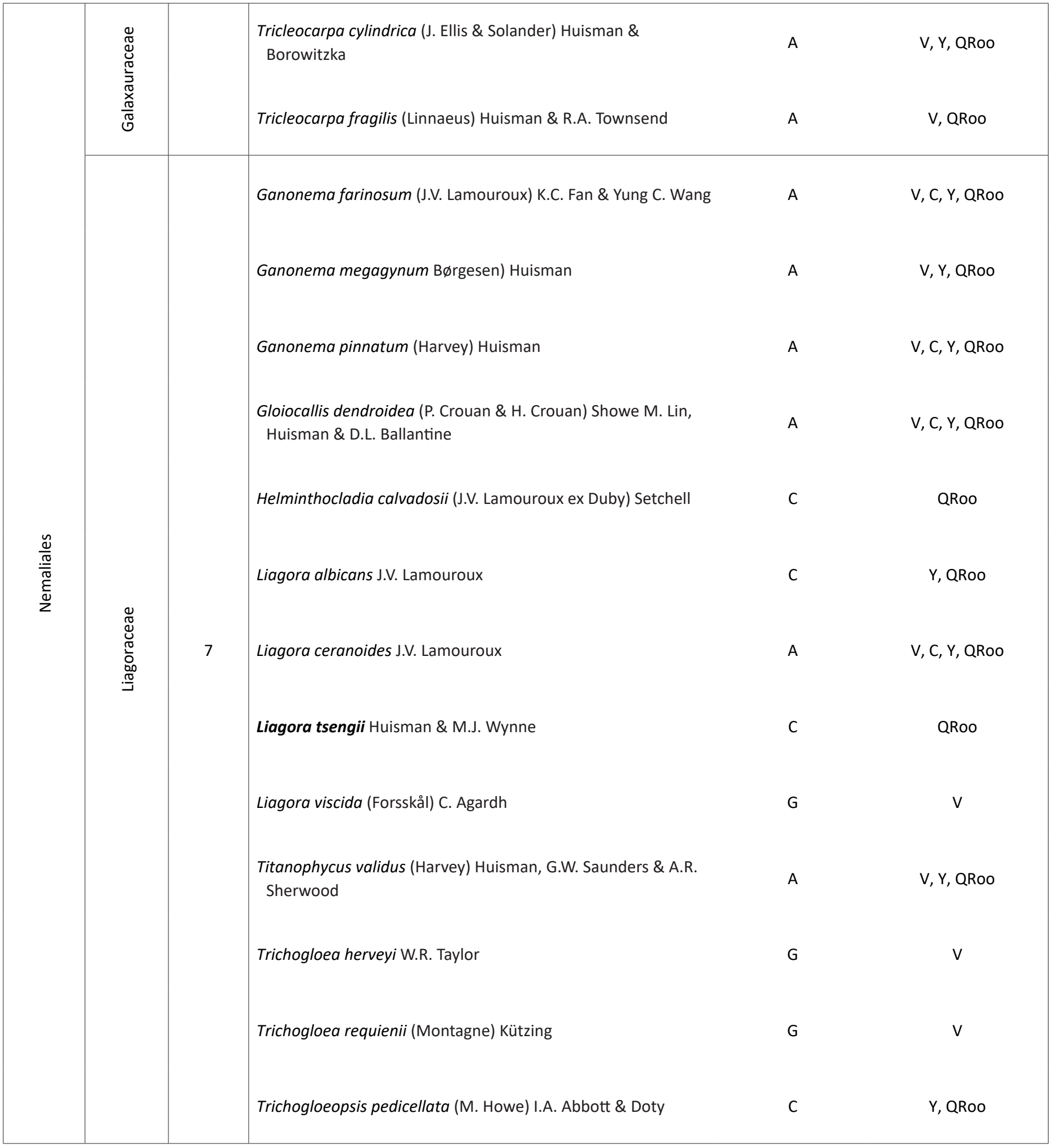


Apéndice 2. Continuación.

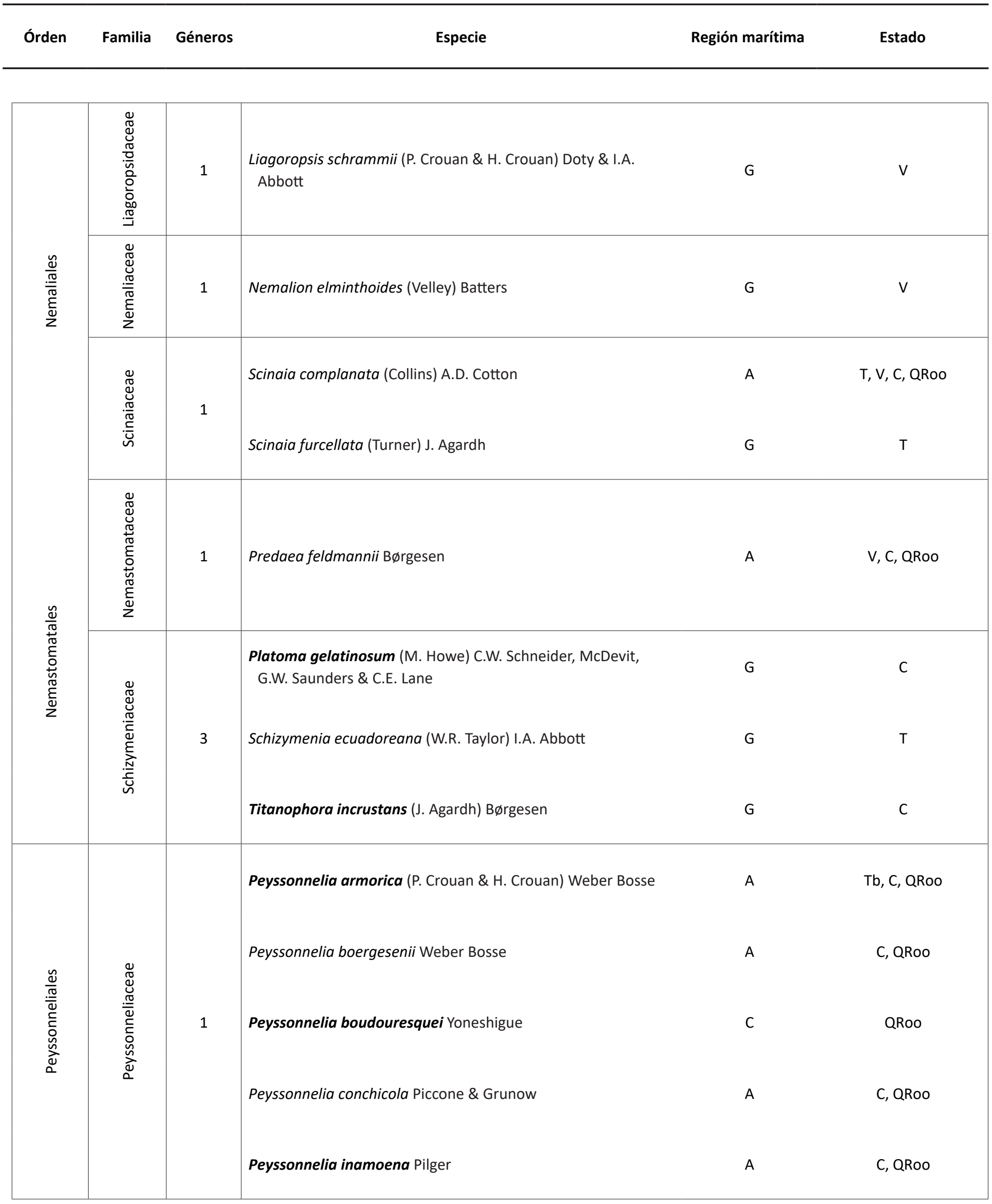


Apéndice 2. Continuación.

\begin{tabular}{|c|c|c|c|c|c|}
\hline \multirow{3}{*}{ 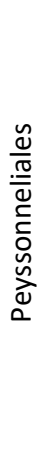 } & \multirow{3}{*}{ 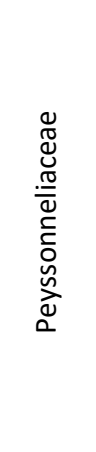 } & & Peyssonnelia nordstedtii Weber Bosse & C & Y, QRoo \\
\hline & & & $\begin{array}{l}\text { Peyssonnelia rubra (Greville) J. Agardh } \\
\text { Peyssonnelia simulans Weber Bosse }\end{array}$ & . & $\begin{array}{l}\text { V, Tb, C, QRoo } \\
\text { QRoo }\end{array}$ \\
\hline & & & Peyssonnelia stoechas Boudouresque \& Denizot & $\mathrm{C}$ & QRoo \\
\hline $\begin{array}{l}\frac{\mathscr{d}}{\frac{\pi}{\tilde{E}}} \\
\frac{\sqrt{0}}{\frac{0}{\alpha}}\end{array}$ & 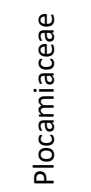 & 1 & Plocamium cartilagineum (Linnaeus) P.S. Dixon & G & V \\
\hline \multirow{5}{*}{ 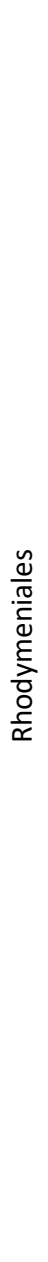 } & 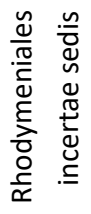 & 1 & Agardhinula browneae (J. Agardh) De Toni & G & C \\
\hline & \multirow{3}{*}{ 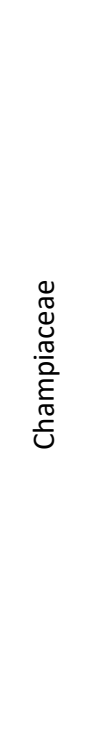 } & \multirow{3}{*}{3} & $\begin{array}{l}\text { Champia minuscula A.B. Joly \& Ugadim } \\
\text { Champia parvula var. parvula (C. Agardh) Harvey }\end{array}$ & . & $\begin{array}{c}\text { QRoo } \\
T, V, C, Y, Q R o o\end{array}$ \\
\hline & & & $\begin{array}{l}\text { Champia parvula var. prostrata L.G. Williams } \\
\text { Champia salicornioides Harvey }\end{array}$ & 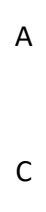 & $\begin{array}{l}\text { V, QRoo } \\
\text { Y, QRoo }\end{array}$ \\
\hline & & & $\begin{array}{l}\text { Coelothrix irregularis (Harvey) Børgesen } \\
\text { Gastroclonium parvum (Hollenberg) C.F. Chang \& B.M. Xia. } \\
\text { Oceanol }\end{array}$ & A & V, Y, QRoo \\
\hline & 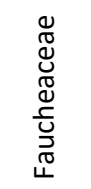 & 2 & $\begin{array}{l}\text { Gloiderma atlanticum (Searles) R.E. Norris } \\
\text { Gloiocladia blomquistii (Searles) R.E. Norris }\end{array}$ & A & C, QRoo \\
\hline
\end{tabular}


Apéndice 2. Continuación.

\begin{tabular}{|c|c|c|c|c|c|}
\hline \multirow{4}{*}{ 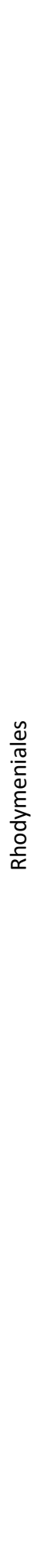 } & 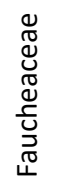 & & Gloiocladia rubrispora (Searles) R.E. Norris & G & C \\
\hline & 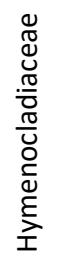 & 1 & Asteromenia peltata (W.R. Taylor) Huisman \& A.J.K. Millar & C & QRoo \\
\hline & 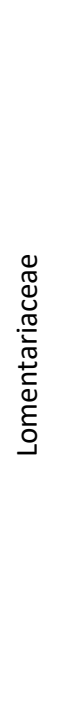 & 3 & $\begin{array}{l}\text { Ceratodictyon intricatum (C. Agardh) R.E. Norris } \\
\text { Ceratodictyon planicaule (W.R. Taylor) M.J. Wynne } \\
\text { Ceratodictyon variabile (J. Agardh) R.E. Norris } \\
\text { Hooperia divaricata (Durant) M.J. Wynne, C.W. Schneider \& } \\
\text { G.W. Saunders } \\
\text { Lomentaria rawitscheri A.B. Joly } \\
\text { Lomentaria uncinata Martens ex Kützing }\end{array}$ & . & $\begin{array}{l}\text { V, C, Y, QRoo } \\
\text { T, C, QRoo } \\
\text { V, Tb, C } \\
\text { C, QRoo } \\
\text { C, QRoo } \\
\text { V, Tb }\end{array}$ \\
\hline & 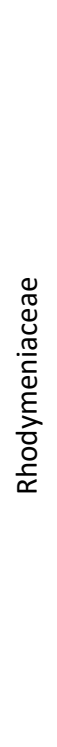 & 5 & $\begin{array}{l}\text { Botryocladia enteromorpha (Harvey) W.E. Schmidt, Lozada- } \\
\text { Troche, D.L. Ballantine \& Fredericq } \\
\text { Botryocladia occidentalis (Børgesen) Kylin } \\
\text { Botryocladia pyriformis (Børgesen) Kylin } \\
\text { Botryocladia spinulifera W.R. Taylor \& I.A. Abbott } \\
\text { Chrysymenia halymenioides Harvey } \\
\text { Chrysymenia planifrons (Melvill) J. Agardh }\end{array}$ & 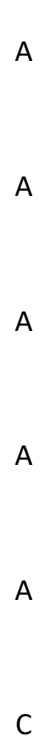 & $\begin{array}{l}\text { C, QRoo } \\
\text { C, QRoo } \\
\text { C, QRoo } \\
\text { QRoo }\end{array}$ \\
\hline
\end{tabular}


Apéndice 2. Continuación.

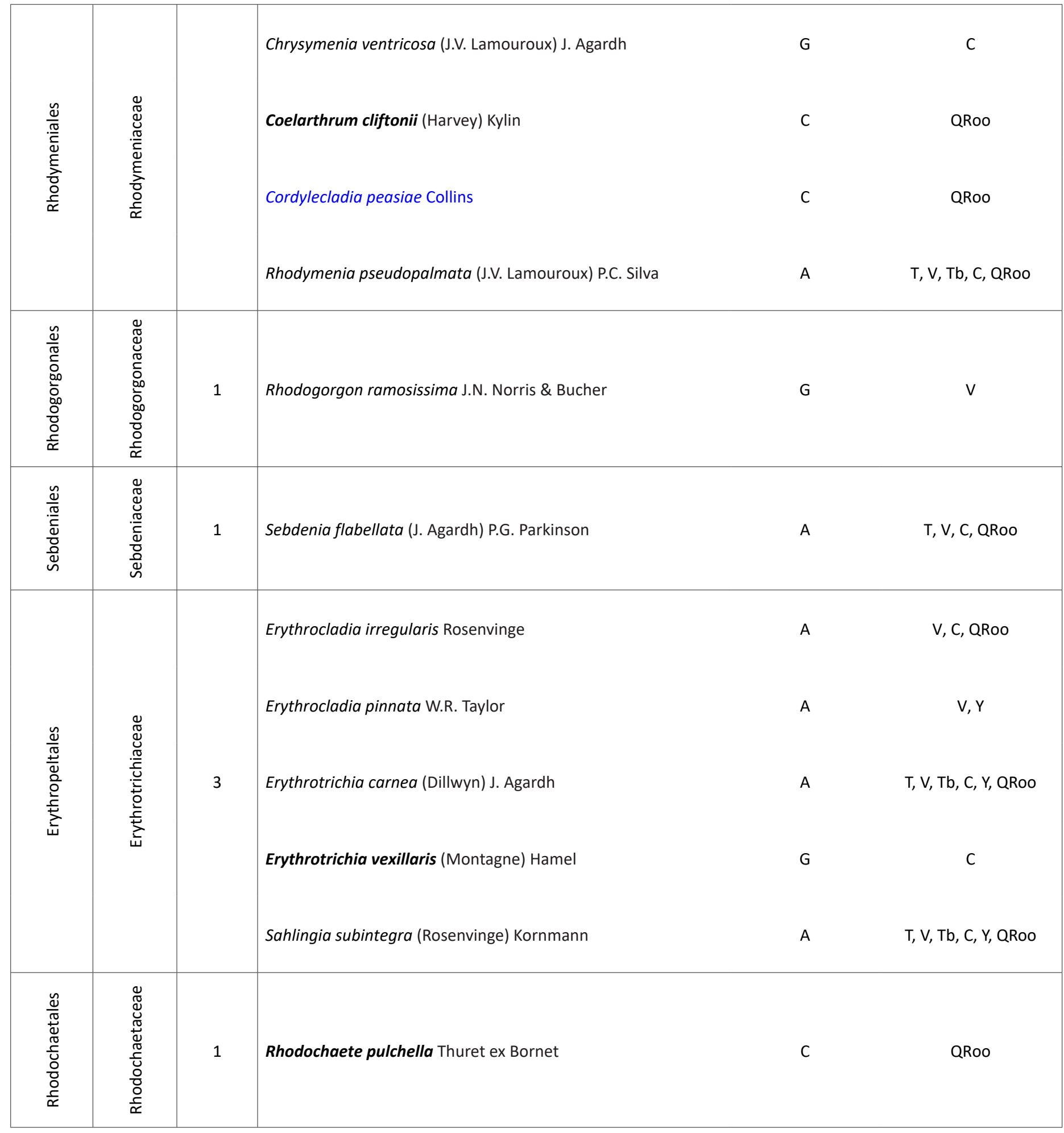


Apéndice 2. Continuación.

\begin{tabular}{|c|c|c|c|c|c|}
\hline Órden & Familia & Géneros & Especie & Región marítima & Estado \\
\hline 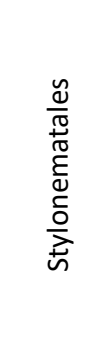 & 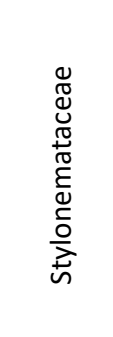 & 3 & $\begin{array}{l}\text { Bangiopsis dumontioides (P. Crouan \& H. Crouan) V. } \\
\text { Krishnamurthy } \\
\text { Chroodactylon ornatum (C. Agardh) Basson } \\
\text { Stylonema alsidii (Zanardini) K.M. Drew }\end{array}$ & 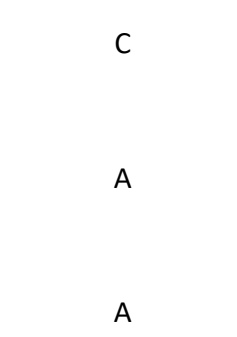 & $\begin{array}{c}\text { QRoo } \\
\text { T, V, Tb, C, Y, QRoo }\end{array}$ \\
\hline
\end{tabular}

